Monte Carlo Variance Reduction Approaches for Non-Boltzmann Tallies

Thomas E. Booth 


\section{DISCLAIMER}

This report was prepared as an account of work sponsored by an agency of the United States Government. Neither the United States Government nor any agency thereof, nor any of their employees, make any warranty, express or implied, or assumes any legal liability or responsibility for the accuracy, completeness, or usefulness of any information, apparatus, product, or process disclosed, or represents that its use would not infringe privately owned rights. Reference herein to any specific commercial product, process, or service by trade name, trademark, manufacturer, or otherwise does not necessarily constitute or imply its endorsement, recommendation, or favoring by the United States Government or any agency thereof. The views and opinions of authors expressed herein do not necessarily state or reflect those of the United States Government or any agency thereof. 


\section{DISCLAIMER}

Portions of this document may be illegible in electronic image products. Images are produced from the best available original document. 


\title{
MONTE CARLO VARIANCE REDUCTION APPROACHES FOR NON-BOLTZMANN TALLIES
}

by

\author{
Thomas E. Booth
}

\begin{abstract}
Quantities that depend on the collective effects of groups of particles cannot be obtained from the standard Boltzmann transport equation. Monte Carlo estimates of these quantities are called non-Boltzmann tallies and have become increasingly important recently. Standard Monte Carlo variance reduction techniques were designed for tallies based on individual particles rather than groups of particles. Experience with non-Boltzmann tallies and analog Monte Carlo has demonstrated the severe limitations of analog Monte Carlo for many non-Boltzmann tallies. In fact, many calculations absolutely require variance reduction methods to achieve practical computation times.

Three different approaches to variance reduction for nonBoltzmann tallies are described and shown to be unbiased. The advantages and disadvantages of each of the approaches are discussed.
\end{abstract}

\section{INTRODUCTION}

Variance reduction techniques have been tremendously successful when applied to Monte Carlo radiation transport codes-so successful, in fact, that every major Monte Carlo radiation transport code contains numerous variance reduction techniques. However, these techniques only apply to estimates depending on a single particle. As a result, estimates (like pulse height spectra) that depend on the collective effects of several particles are restricted to analog Monte Carlo. This restriction is so severe that many calculations are not even attempted.

Many scientific experiments depend on the collective transport of several particles. For example, there are large numbers of neutron coincidence experiments in 
which a neutron can only be counted if it is detected in coincidence with another neutron. Another example is pulse height spectra experiments. In these experiments the detector has a number of channels, each covering a different range of total deposited energy. If two neutrons from the same fission each deposit .5 MeV, then the detector must count 1 in the $1 \mathrm{MeV}$ channel and not 2 in the $.5 \mathrm{MeV}$ channel. Current Monte Carlo codes have extreme difficulty trying to calculate some of these experiments because no theoretical basis exists for applying variance reduction techniques and thus the calculations must be done with analog Monte Carlo.

This report describes three approaches to variance reduction for non-Boltzmann tallies:

1. the deconvolution approach (Section II)

2. the corrected single particle approach (Section III)

3. the supertrack approach (Section IV)

The deconvolution approach applies single particle variance reduction methods to each particle of a collection and then analyzes (deconvolutes) how the distribution of the collection of particles has been modified and weights the tallies appropriately. The supertrack approach applies variance reduction to collections of tracks (supertracks) and requires redefinition of standard Monte Carlo terms. For example, the individual particle tracks would no longer carry any weight; the variance reduction is applied to the supertracks, and thus the weights are associated with the supertracks. The corrected single particle approach is perhaps the most difficult. In this approach, the tracks are first treated as single particles with the traditional single particle weights, and then the collective effects are introduced by estimating the difference between transporting the particles as a collection and transporting the particles individually.

Before proceeding to non-Boltzmann tallies, it is probably useful to review an analog random walk with a non-Boltzmann tally. A pulse height tally will be chosen as an example. Figure 1 shows an analog random walk with four physical branch nodes. Each of these physical branch nodes will be called a physical split and labeled with a P. Each $E_{k}$ listed beside a branch is the energy the photon loses in the detector between the upper and lower nodes of the branch. For example, $E_{8}$ is the energy the right branch photon of node 4 loses in the detector between node 4 and the termination of the right branch. A pulse height detector responds to the total energy lost (per source particle) in the detector by all photons in a history. In Fig. 1, the total energy lost is $E_{1}+\cdots+E_{10}$. Note that there may be many scatterings between branch nodes. The straight lines between nodes do not indicate a collisionless free-flight between nodes. Scatterings are not explicitly shown in Fig. 1. This means, for example, that $E_{8}$ may be the total energy lost in the detector between node 4 and termination in ten collisions. 


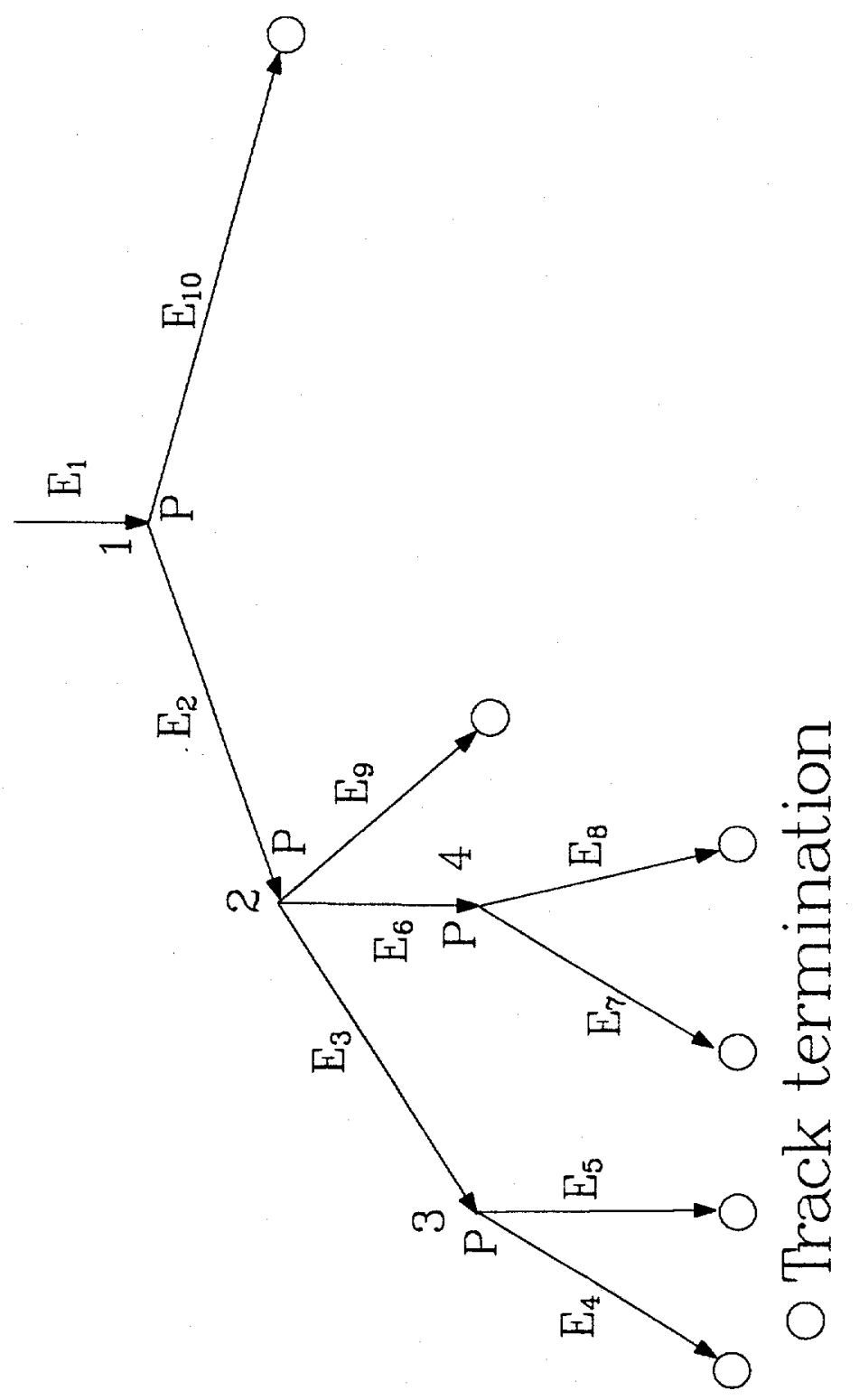




\section{NON-BOLTZMANN TALLIES WITH SPLITTING USING THE DECONVOLUTION APPROACH}

One natural variance reduction approach for non-Boltzmann tallies is to keep the same random walk sampling procedure used for Boltzmann tallies and simply ask what the correct non-Boltzmann tallies are for any given completed random walk. For simplicity, this section considers applying simple integer splitting (no Russian roulette) techniques to a single photon pulse height tally corresponding to a single detector.

Consider a pulse height tally with integer splitting. A typical random walk is shown in Fig. 2. The photon starts at the top of the figure and has various branches below it. These branches are labeled with sequential integers in the order in which the branches occur in the random walk. Some of the branches are physical branches (e.g., pair production gammas or double fluorescence) and some are variance reduction branches. The physical branches are labeled with $\mathrm{P}$ and the variance reduction branches are labeled with $V$.

Note that a variance reduction split is a mathematical artifice and not a physical reality. That is, it is physically impossible for a particle to take more than one branch of a variance reduction split. A variance reduction split should be thought of as a set of possibilities for what might occur subsequent to the split. In reality, a physical particle will take exactly one branch. Thus, physically possible random walks can be produced from Fig. 2 by sampling one branch of each variance reduction split in the tree. In fact, this sampling essentially "undoes" the variance reduction splits, and thus a physically realizable random walk is produced.

For example, choosing the middle branch at node 2, the right branch at node 5 , and the left branch at node 8 would occur with probability $\frac{1}{3} \frac{1}{2} \frac{1}{2}=\frac{1}{12}$ and would result in the physically realizable tree of Fig. 3 . That is, an energy of $E_{1}+E_{2}+$ $E_{6}+E_{7}+E_{8}+E_{10}+E_{14}+E_{15}+E_{16}+E_{18}$ is deposited in the detector (from the tree of Fig. 2) with probability $\frac{1}{12}$. Thus, if the variance reduction branches of Fig. 2 are randomly sampled, then a tally of 1 will be posted in the energy bin containing $E_{1}+E_{2}+E_{6}+E_{7}+E_{8}+E_{10}+E_{14}+E_{15}+E_{16}+E_{18}$ with probability $\frac{1}{12}$. Note that rather than randomly sampling for the tree of Fig. 3 to occur, one can always have the tree occur with probability 1 and assign a statistical weight $w$ of

$$
w=\frac{\text { true probability }}{\text { sampled probability }}=\frac{\frac{1}{12}}{1}=\frac{1}{12}
$$

In fact, this can be done with every possible physical tree that might occur by sampling Fig. 2. That is, make each of the possible physical trees occur with probability 1 and weight each of them appropriately. This approach will be used below and $p$ can be interpreted either as the probability that a given tally occurs in an analog game or as the weight assigned to a tally in the deconvolution procedure.

For simple trees it is easy to enumerate all possible physical random walks existing in the trees, but some thought is required to design a computer algorithm that works for all trees. The algorithm will now be applied to the tree of Fig. 2. The process starts with the highest numbered node; in this case node 8 . Node 8 is a variance reduction split so the energy deposited below node 8 is either 


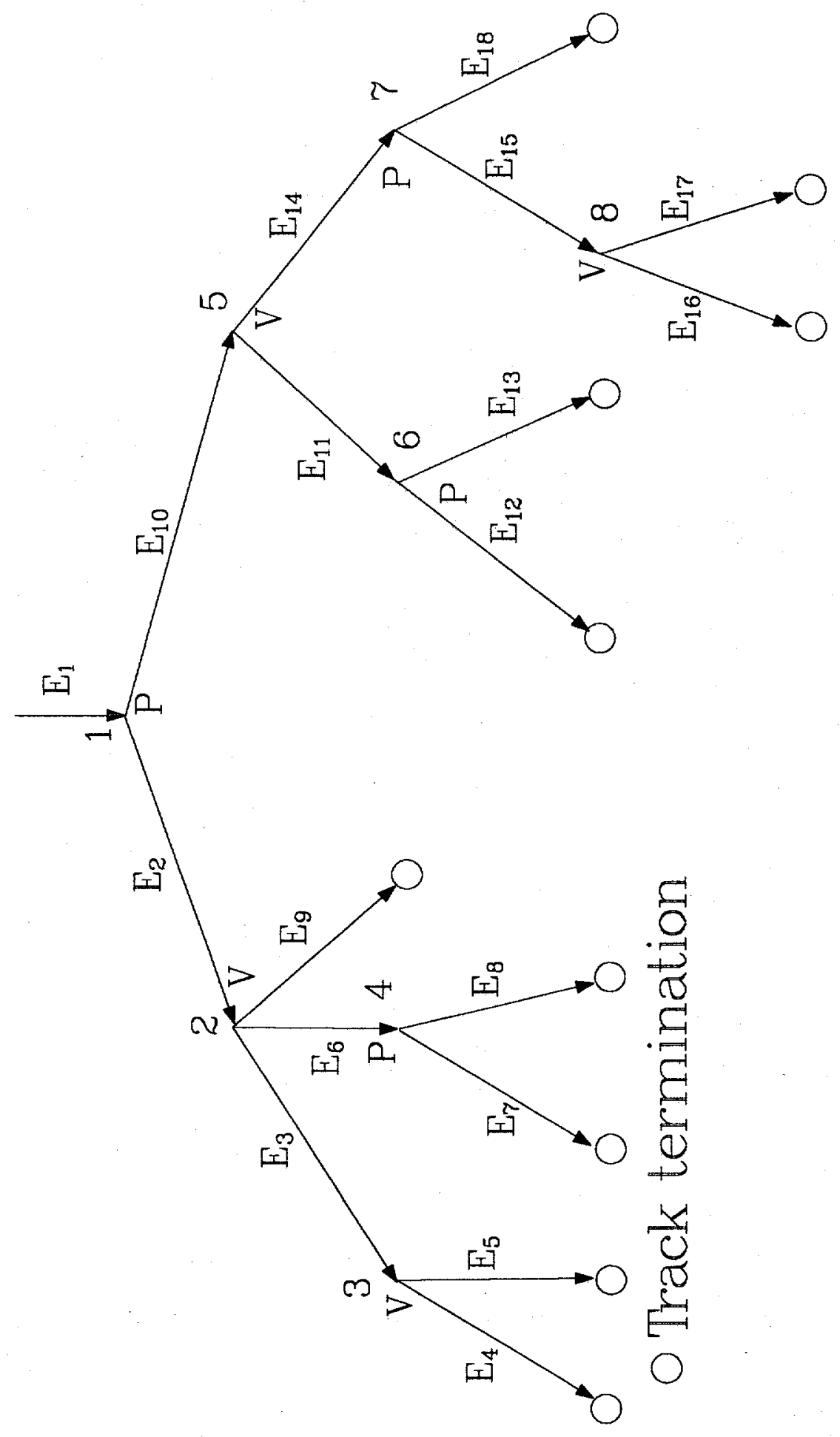

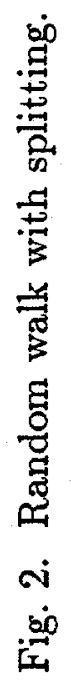




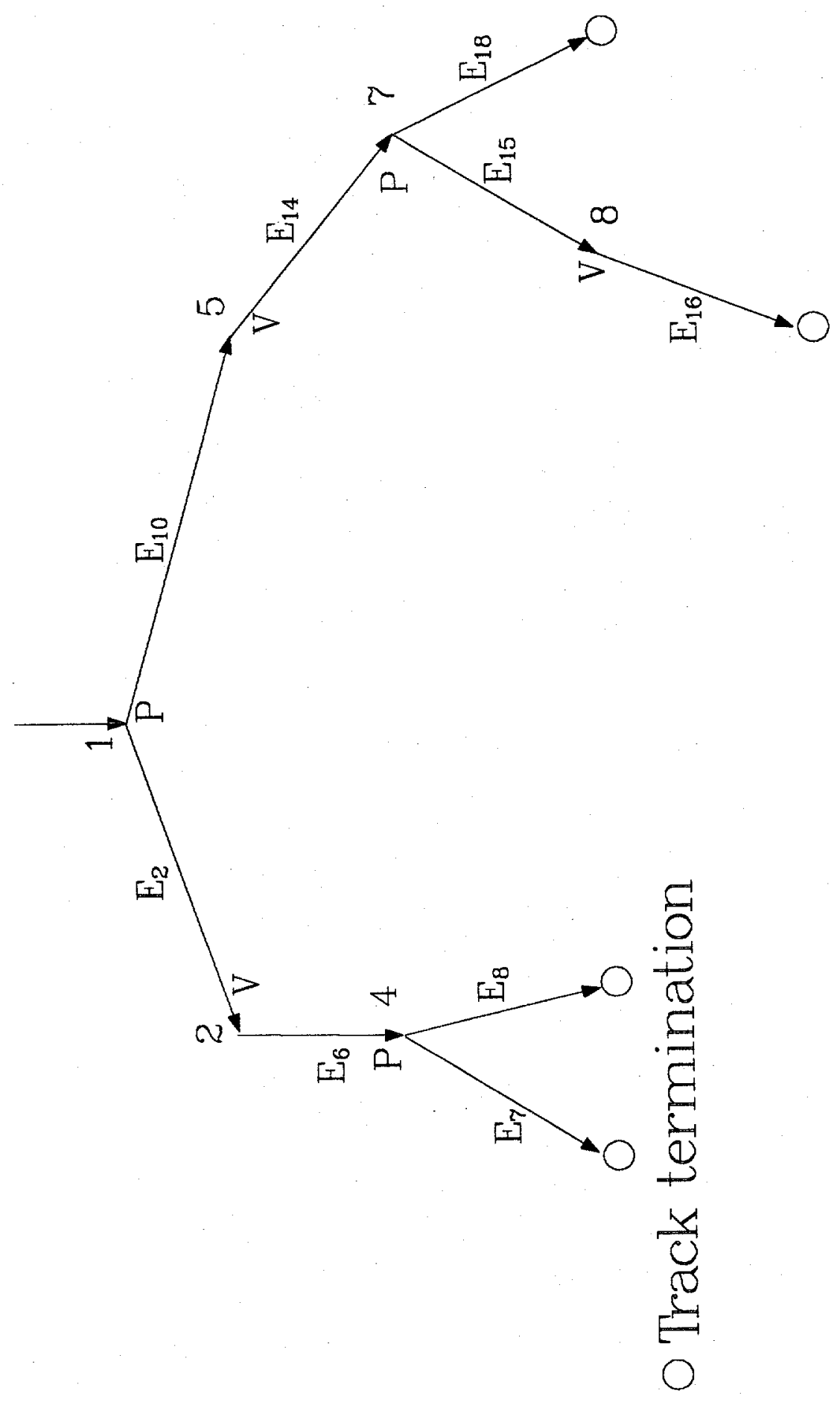

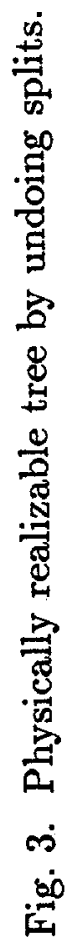




$$
E_{\text {node8 }}=E_{16} \quad p=\frac{1}{2}
$$

or

$$
E_{\text {node8 }}=E_{17} \quad p=\frac{1}{2}
$$

These choices are shown in Fig. 4. (The big circles indicate that the possible values for energy deposited below that node have been enumerated.) Node 7 is a physical split so both branches occur together with probability 1 . That is, the energy deposited below node 7 is $E_{15}+E_{18}+E_{\text {node8 }}$. There are two choices for $E_{\text {node8 }}$ (using Eqs. (2.1) and (2.2)) so the energy deposited below node 7 is either

or

$$
E_{\text {node } 7}=E_{15}+E_{16}+E_{18} \quad p=\frac{1}{2}
$$

$$
E_{\text {node } 7}=E_{15}+E_{17}+E_{18} \quad p=\frac{1}{2}
$$

These choices are shown in Fig. 5. Node 6 is a physical split and thus both branches occur together so the energy deposited below node 6 is

$$
E_{\text {node6 }}=E_{12}+E_{13} \quad p=1
$$

These choices are shown in Fig. 6 . Node 5 is a variance reduction split so the energy deposited below node 5 is either

or

$$
E_{\text {node5 }}=E_{11}+E_{\text {node6 }} \quad p=\frac{1}{2}
$$

$$
E_{\text {node5 }}=E_{14}+E_{\text {node7 }} \quad p=\frac{1}{2}
$$

Using Eq. (2.5) in Eq. (2.6) and Eqs. (2.3-2.4) in Eq. (2.7) yields

$$
E_{n o d e 5}=E_{11}+E_{12}+E_{13} \quad p=\frac{1}{2}
$$

or

$$
E_{n o d e 5}=E_{14}+E_{15}+E_{16}+E_{18} \quad p=\frac{1}{4}
$$

or

$$
E_{\text {node } 5}=E_{14}+E_{15}+E_{17}+E_{18} \quad p=\frac{1}{4}
$$

These choices are shown in Fig. 7. Node 4 is a physical split and thus both branches occur together so the energy deposited below node 4 is 


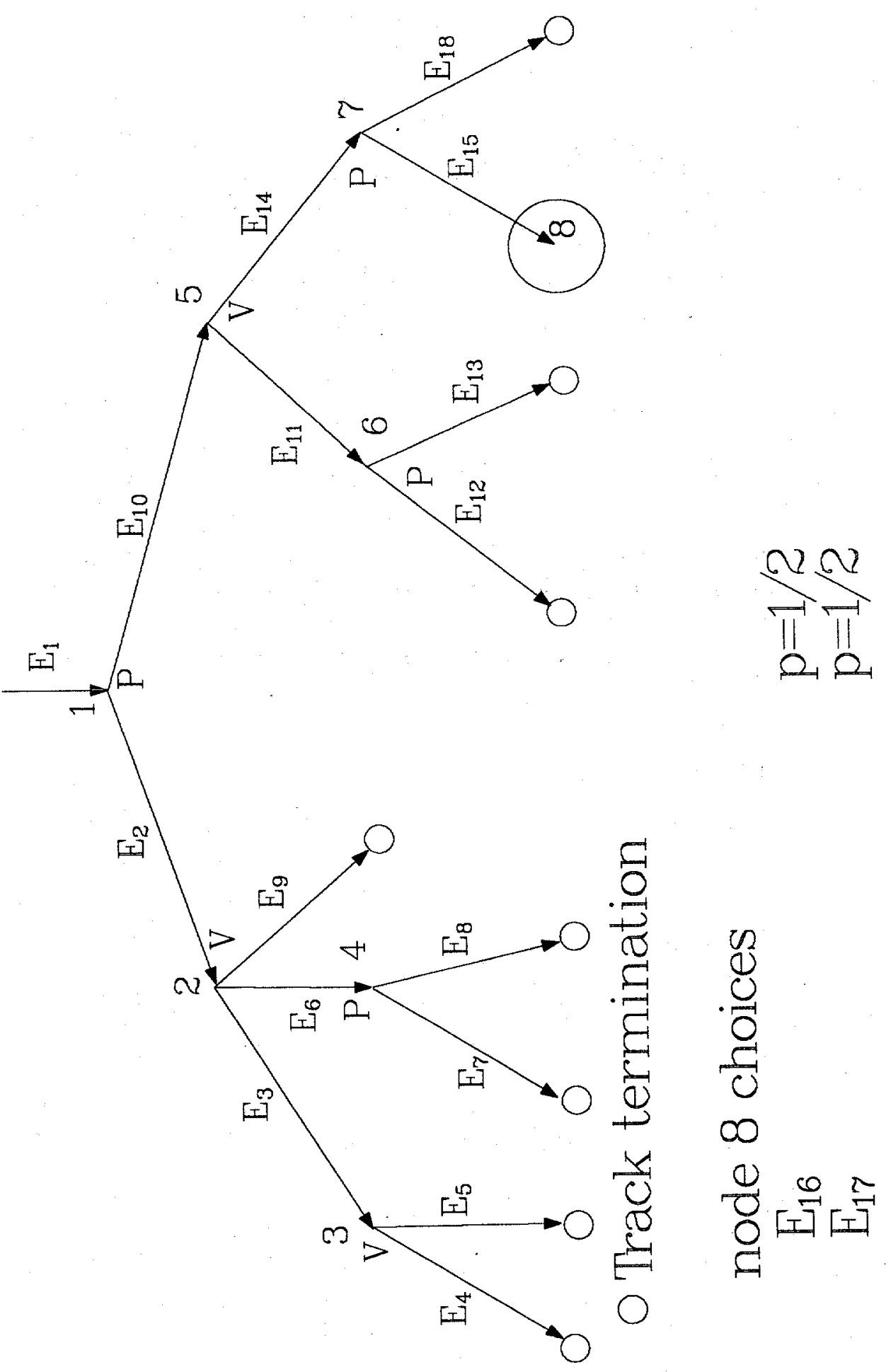




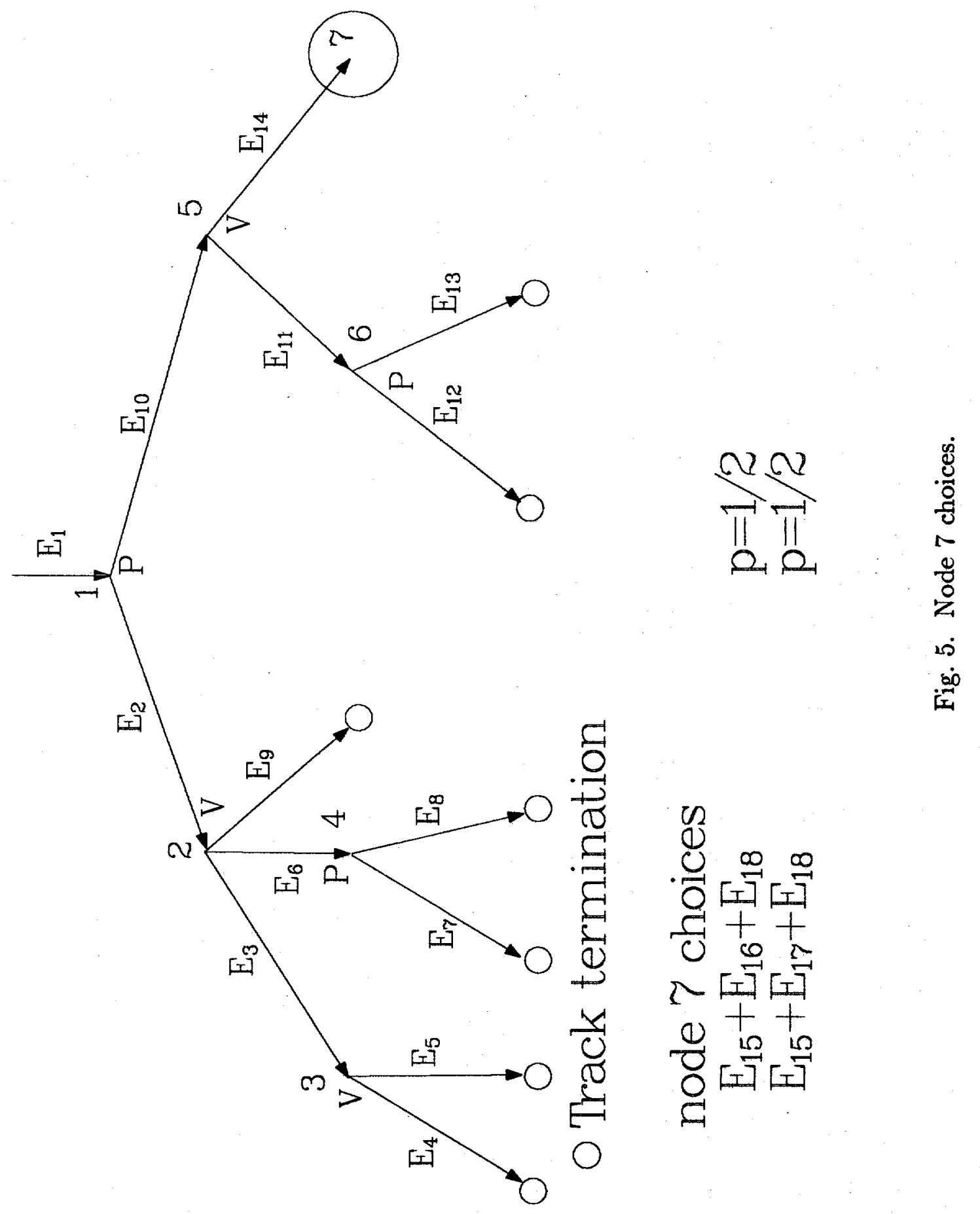



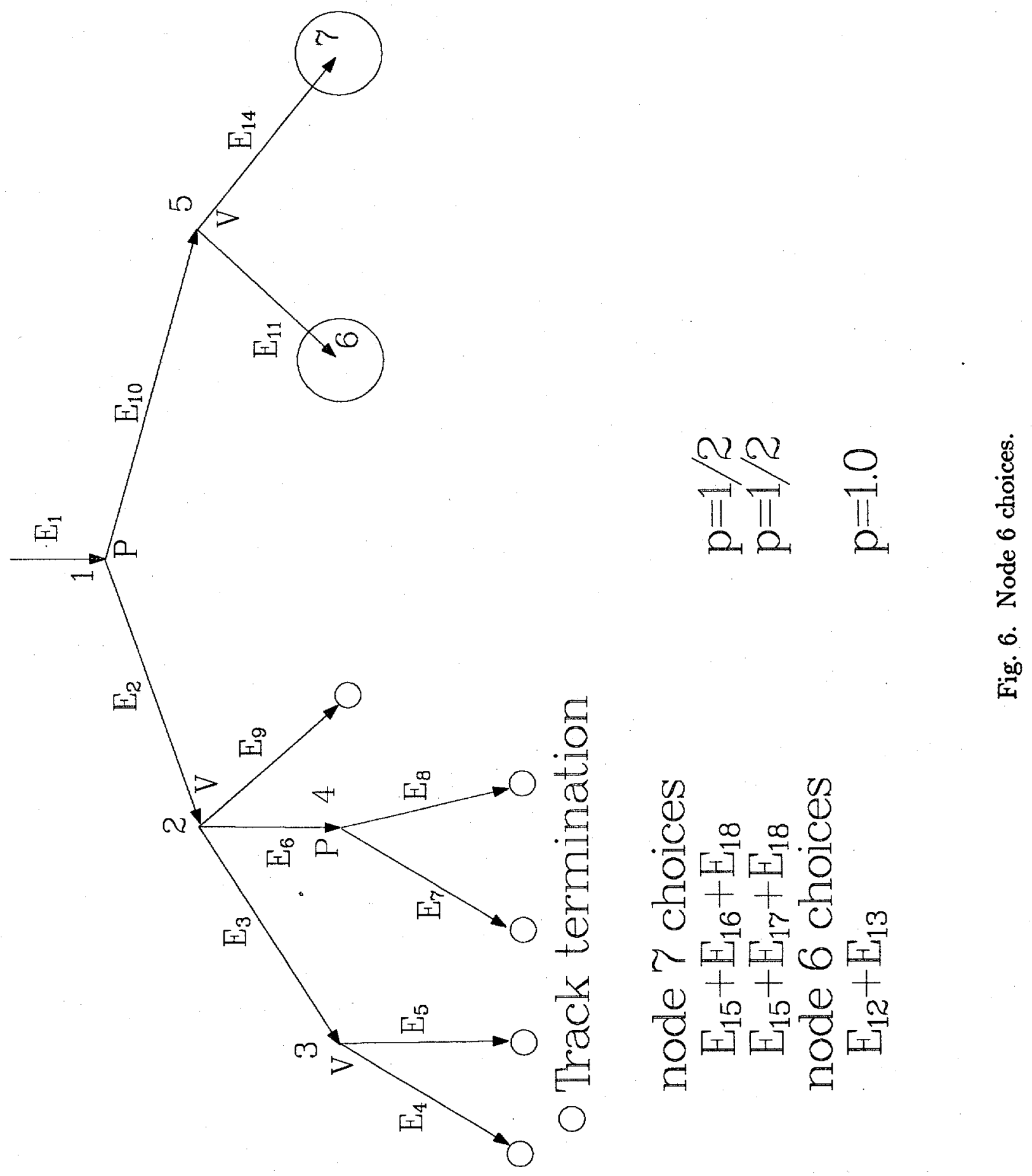


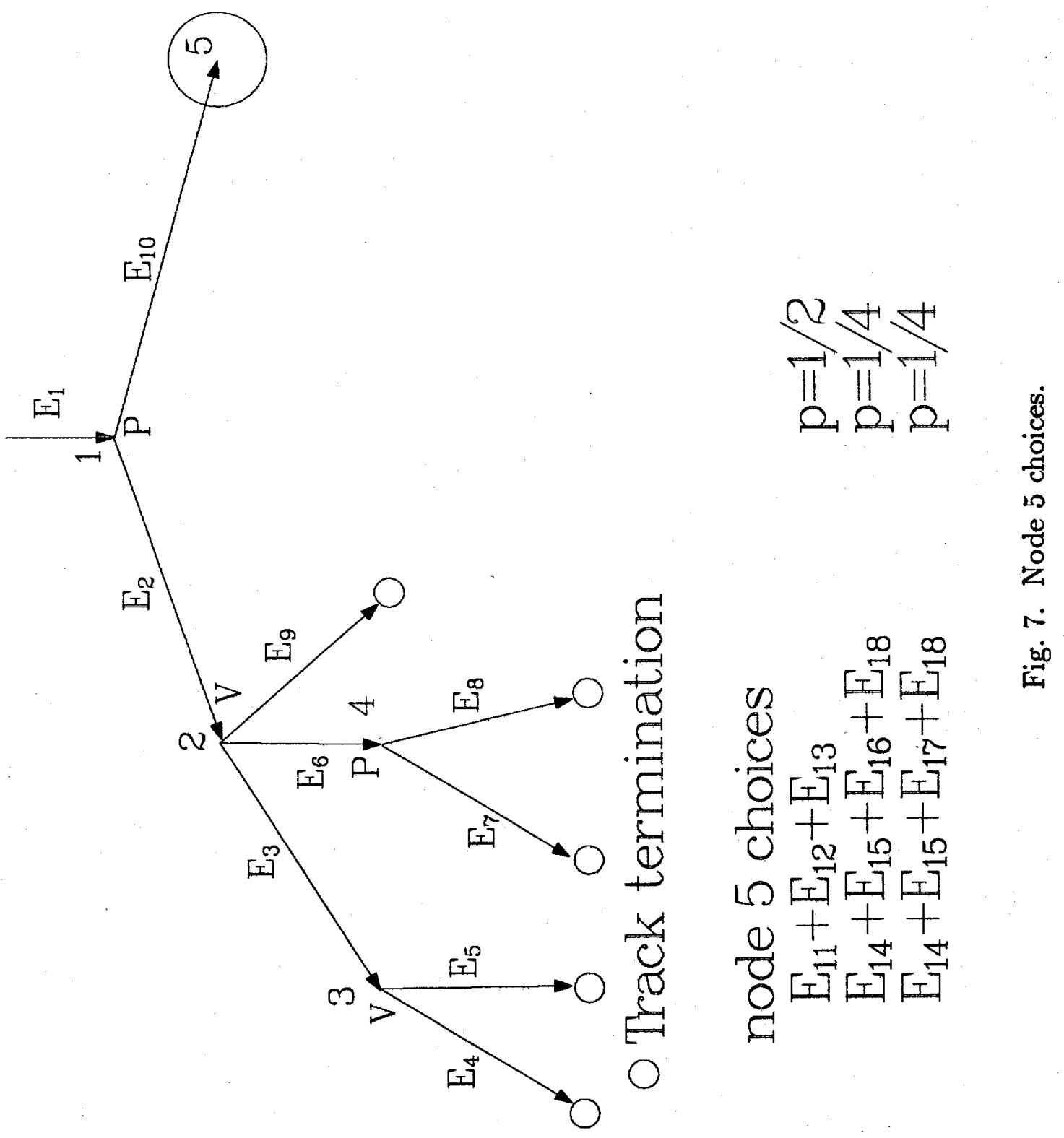




$$
E_{\text {node4 }}=E_{7}+E_{8} \quad p=1
$$

These choices are shown in Fig. 8 . Node 3 is a variance reduction split so the energy deposited below node 3 is either

or

$$
E_{\text {node } 3}=E_{4} \quad p=\frac{1}{2}
$$

$$
E_{\text {node } 3}=E_{5} \quad p=\frac{1}{2}
$$

These choices are shown in Fig. 9. Node 2 is a variance reduction split so the energy deposited below node 2 is either

$$
E_{\text {node } 2}=E_{3}+E_{\text {node3 } 3} \quad p=\frac{1}{3}
$$

or

$$
E_{\text {node } 2}=E_{6}+E_{\text {node4 }} \quad p=\frac{1}{3}
$$

or

$$
E_{\text {node } 2}=E_{9} \quad p=\frac{1}{3}
$$

Using Eqs. (2.12-2.13) in Eq. (2.14) yields

$$
E_{n o d e 2}=E_{3}+E_{4} \quad p=\frac{1}{6}
$$

or

$$
E_{n o d e 2}=E_{3}+E_{5} \quad p=\frac{1}{6}
$$

Using Eq. (2.11) in Eq. (2.15) yields

$$
E_{\text {node } 2}=E_{6}+E_{7}+E_{8} \quad p=\frac{1}{3}
$$

Repeating Eq. (2.16) for convenience

$$
E_{\text {node2 }}=E_{9} \quad p=\frac{1}{3}
$$

These choices are shown in Fig. 10. Node 1 is a physical split and thus both branches occur together so the energy deposited below node 1 is

$$
E_{\text {node } 1}=E_{2}+E_{10}+E_{\text {node2 } 2}+E_{\text {node } 5} \quad p=1
$$




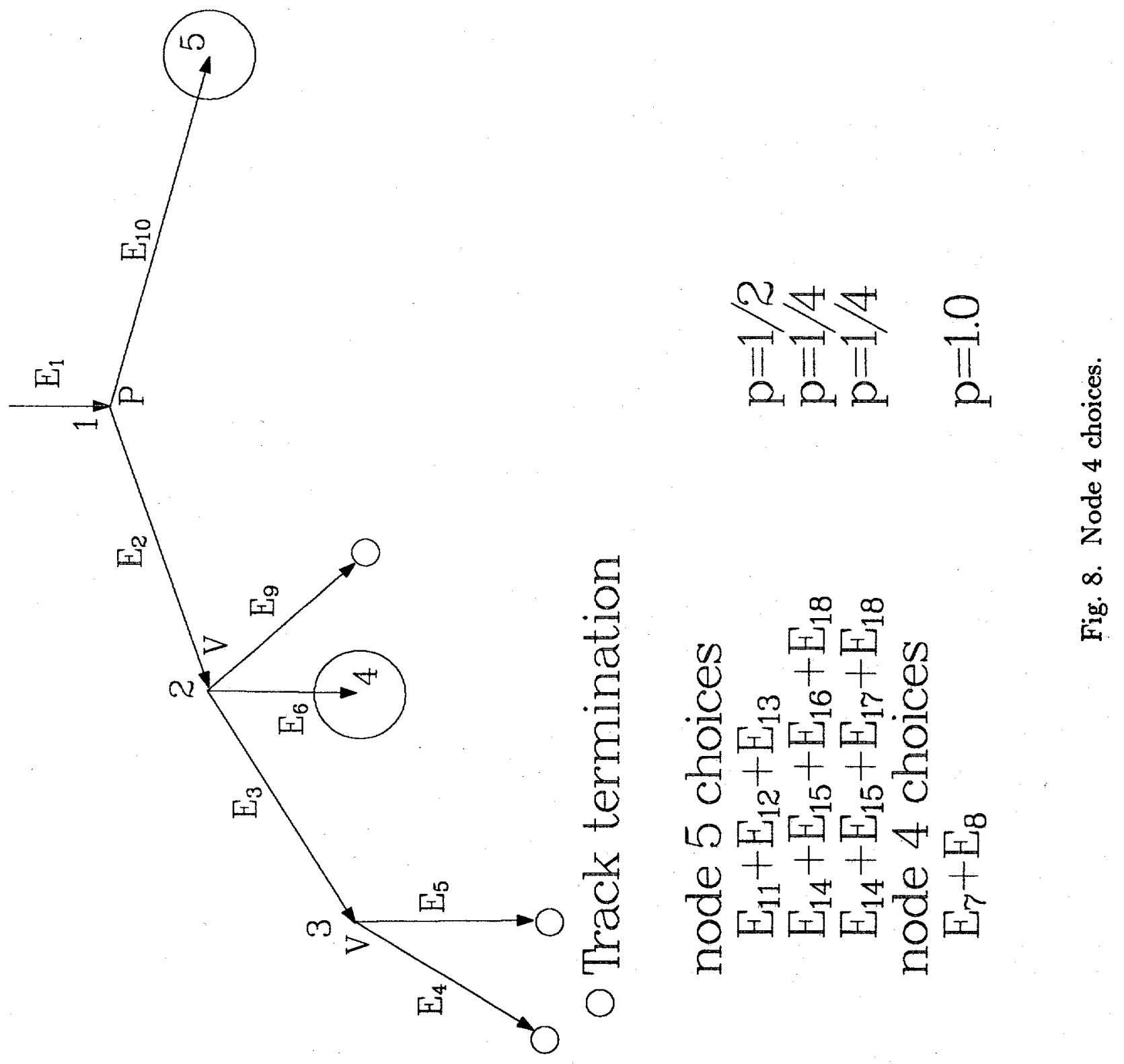



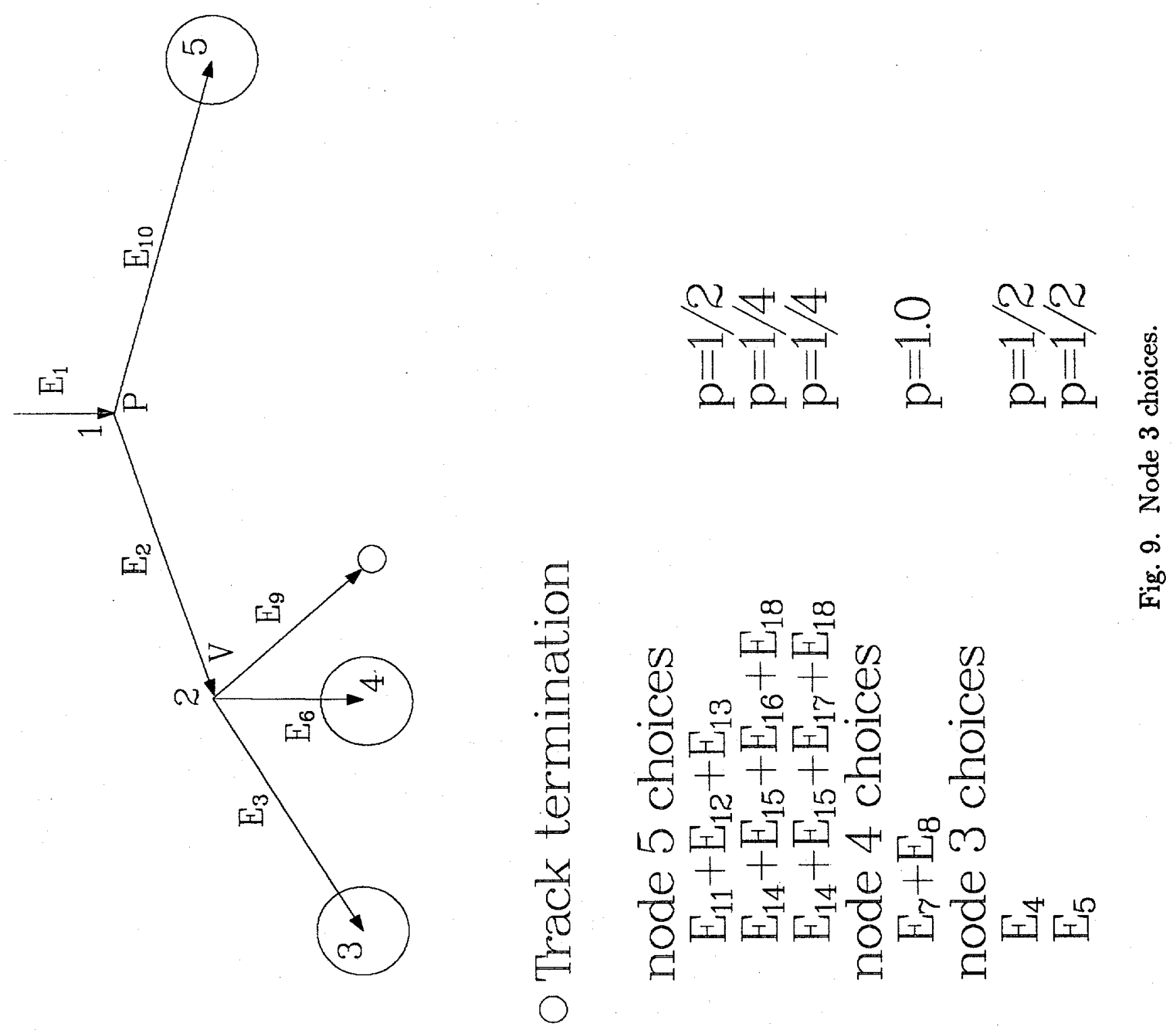


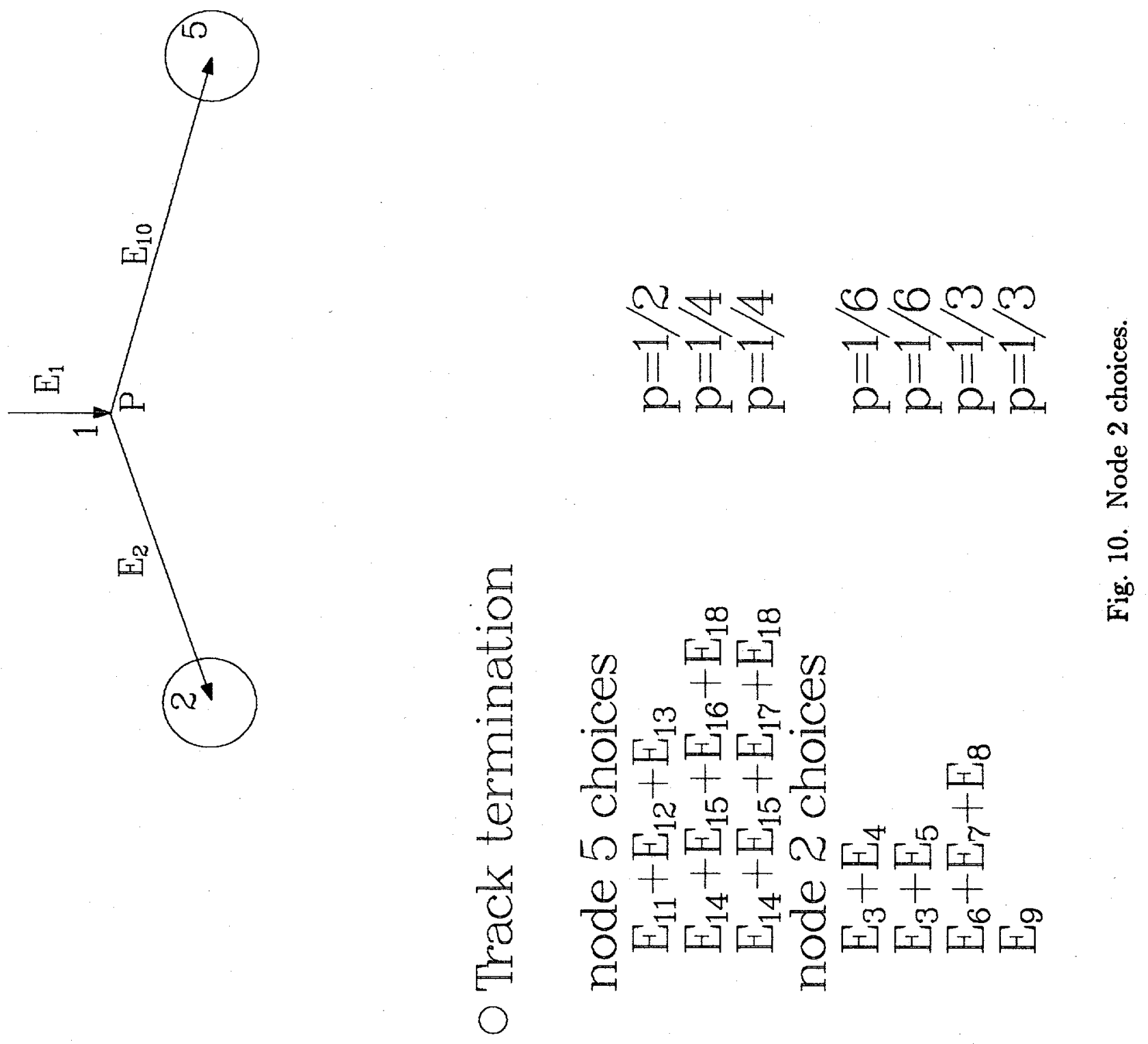


From Eqs. (2.8-2.10) it follows that

$$
E_{\text {node } 1}=E_{2}+E_{10}+E_{\text {node2 }}+E_{11}+E_{12}+E_{13} \quad p=\frac{1}{2}
$$

or

$$
E_{n o d e 1}=E_{2}+E_{10}+E_{n o d e 2}+E_{14}+E_{15}+E_{16}+E_{18} \quad p=\frac{1}{4}
$$

or

$$
E_{\text {node } 1}=E_{2}+E_{10}+E_{\text {node } 2}+E_{14}+E_{15}+E_{17}+E_{18} \quad p=\frac{1}{4}
$$

Substituting Eq. (2.17) into Eqs. (2.22-2.24)

$$
E_{n o d e 1}=E_{2}+E_{10}+E_{3}+E_{4}+E_{11}+E_{12}+E_{13} \quad p=\frac{1}{12}
$$

or

or

$$
E_{n o d e 1}=E_{2}+E_{10}+E_{3}+E_{4}+E_{14}+E_{15}+E_{16}+E_{18} \quad p=\frac{1}{24}
$$

$$
E_{\text {node1 } 1}=E_{2}+E_{10}+E_{3}+E_{4}+E_{14}+E_{15}+E_{17}+E_{18} \quad p=\frac{1}{24}
$$

Substituting Eq. (2.18) into Eqs. (2.22-2.24)

$$
E_{n o d e 1}=E_{2}+E_{10}+E_{3}+E_{5}+E_{11}+\dot{E}_{12}+E_{13} \quad p=\frac{1}{12}
$$

or

or

$$
E_{n o d e 1}=E_{2}+E_{10}+E_{3}+E_{5}+E_{14}+E_{15}+E_{16}+E_{18} \quad p=\frac{1}{24}
$$

$$
E_{\text {node } 1}=E_{2}+E_{10}+E_{3}+E_{5}+E_{14}+E_{15}+E_{17}+E_{18} \quad p=\frac{1}{24}
$$

Substituting Eq. (2.19) into Eqs. (2.22-2.24)

$$
E_{n o d e 1}=E_{2}+E_{10}+E_{6}+E_{7}+E_{8}+E_{11}+E_{12}+E_{13} \quad p=\frac{1}{6}
$$

or

$$
E_{n o d e 1}=E_{2}+E_{10}+E_{6}+E_{7}+E_{8}+E_{14}+E_{15}+E_{16}+E_{18} \quad p=\frac{1}{12}
$$

or

$$
E_{n o d e 1}=E_{2}+E_{10}+E_{6}+E_{7}+E_{8}+E_{14}+E_{15}+E_{17}+E_{18} \quad p=\frac{1}{12}
$$

Substituting Eq. (2.20) into Eqs. (2.22-2.24)

$$
E_{n o d e 1}=E_{2}+E_{10}+E_{9}+E_{11}+E_{12}+E_{13} \quad p=\frac{1}{6}
$$

or 
or

$$
E_{n o d e 1}=E_{2}+E_{10}+E_{9}+E_{14}+E_{15}+E_{16}+E_{18} \quad p=\frac{1}{12}
$$

$$
E_{n o d e 1}=E_{2}+E_{10}+E_{9}+E_{14}+E_{15}+E_{17}+E_{18} \quad p=\frac{1}{12}
$$

Equations (2.25-2.36) are shown on Fig. 11. The energy deposited below node 0 is the same as below node 1 except that $E_{1}$ must be added to the node 0 energy deposited. Thus, using Eqs. (2.25-2.36) yields

or

$$
E_{\text {nodeo }}=E_{1}+E_{2}+E_{10}+E_{3}+E_{4}+E_{11}+E_{12}+E_{13} \quad p=\frac{1}{12}
$$

$$
E_{\text {node } 0}=E_{1}+E_{2}+E_{10}+E_{3}+E_{4}+E_{14}+E_{15}+E_{16}+E_{18} \quad p=\frac{1}{24}
$$

or

$$
\begin{array}{cc}
E_{\text {node } 0}=E_{1}+E_{2}+E_{10}+E_{3}+E_{4}+E_{14}+E_{15}+E_{17}+E_{18} & p=\frac{1}{24} \\
E_{\text {nodeo }}=E_{1}+E_{2}+E_{10}+E_{3}+E_{5}+E_{11}+E_{12}+E_{13} & p=\frac{1}{12}
\end{array}
$$

or

or

$$
E_{\text {node } 0}=E_{1}+E_{2}+E_{10}+E_{3}+E_{5}+E_{14}+E_{15}+E_{16}+E_{18} \quad p=\frac{1}{24}
$$

$$
\begin{array}{ll}
E_{\text {node } 0}=E_{1}+E_{2}+E_{10}+E_{3}+E_{5}+E_{14}+E_{15}+E_{17}+E_{18} & p=\frac{1}{24} \\
E_{\text {node } 0}=E_{1}+E_{2}+E_{10}+E_{6}+E_{7}+E_{8}+E_{11}+E_{12}+E_{13} & p=\frac{1}{6}
\end{array}
$$

or

or

$$
E_{n o d e 0}=E_{1}+E_{2}+E_{10}+E_{6}+E_{7}+E_{8}+E_{14}+E_{15}+E_{16}+E_{18} \quad p=\frac{1}{12}
$$

$$
\begin{array}{rr}
E_{\text {node } 0}=E_{1}+E_{2}+E_{10}+E_{6}+E_{7}+E_{8}+E_{14}+E_{15}+E_{17}+E_{18} & p=\frac{1}{12} \\
E_{n o d e 0}=E_{1}+E_{2}+E_{10}+E_{9}+E_{11}+E_{12}+E_{13} & p=\frac{1}{6}
\end{array}
$$

or

or

$$
E_{\text {node } 0}=E_{1}+E_{2}+E_{10}+E_{9}+E_{14}+E_{15}+E_{16}+E_{18} \quad p=\frac{1}{12}
$$

$$
E_{n o d e 0}=E_{1}+E_{2}+E_{10}+E_{9}+E_{14}+E_{15}+E_{17}+E_{18} \quad p=\frac{1}{12}
$$

These node 0 choices are shown in Fig. 12. 

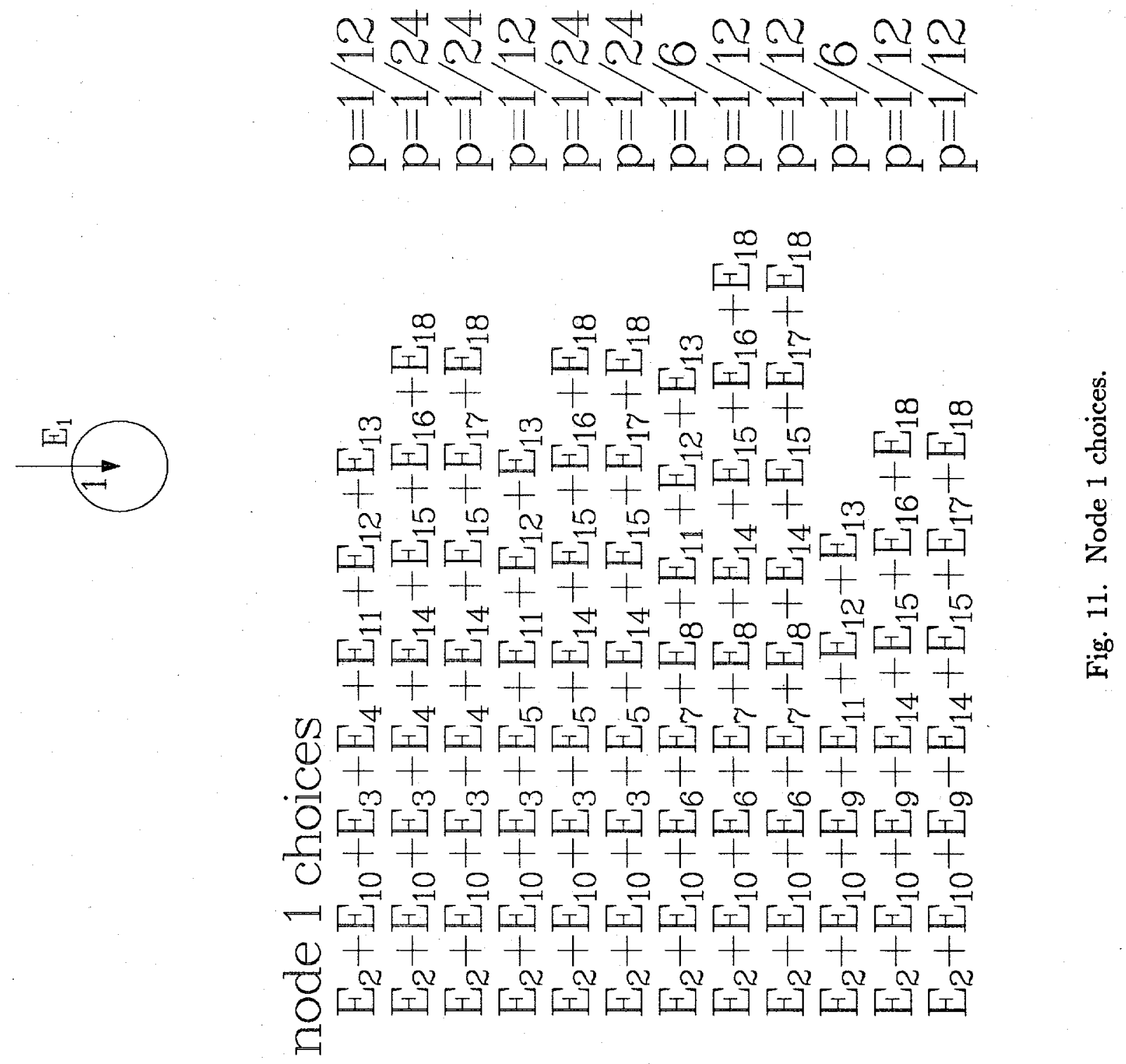


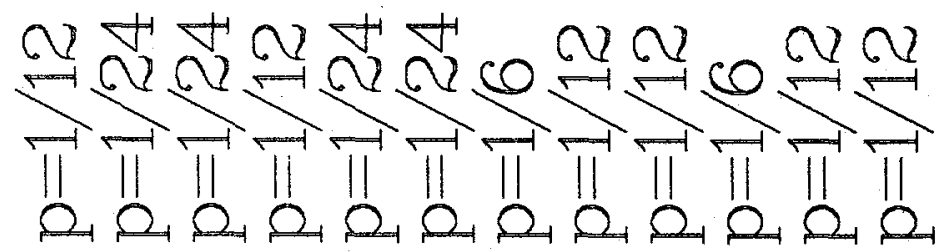

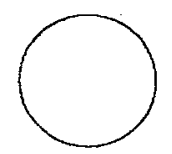

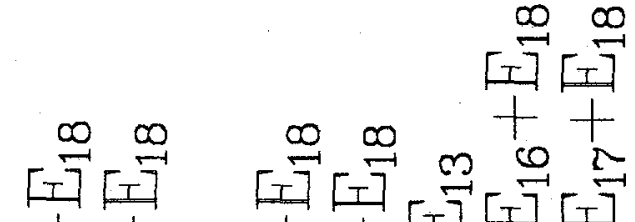

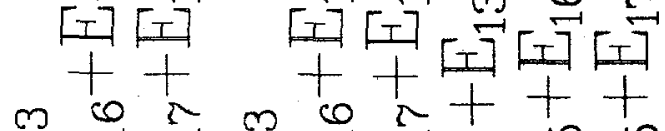

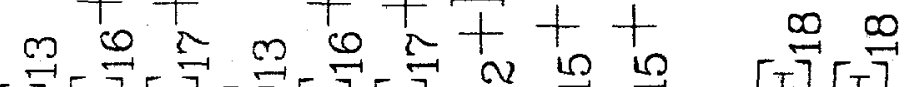

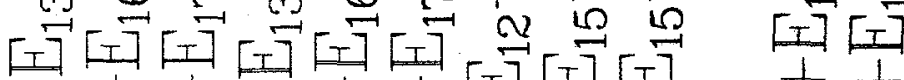

$++++++[I] I I I I+t$

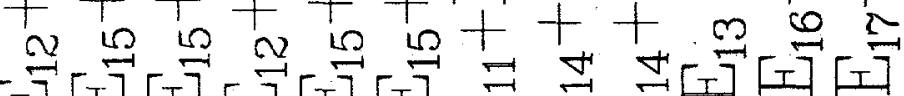

[I] [I] [I] [I] [I] + II II]

$+\leftarrow++++\infty+\infty$ ت

$+++++++++++$

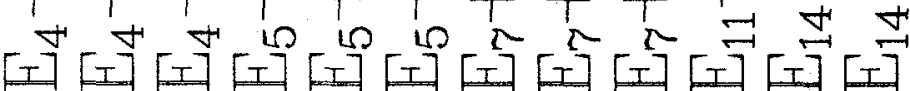

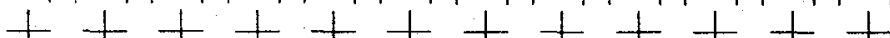

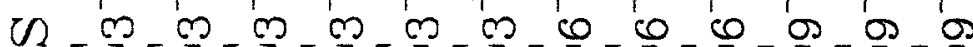

(1) [I] [I] [I] [I] [I] [I] [I] [I] [I] [I] [I] [I] $\mathrm{C}++++++++++$

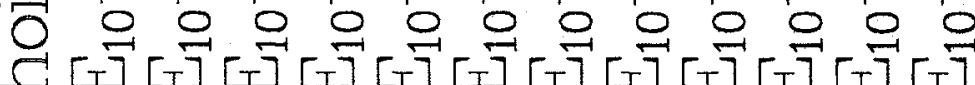

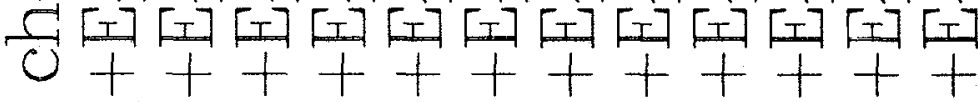

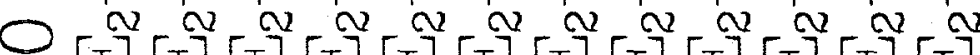

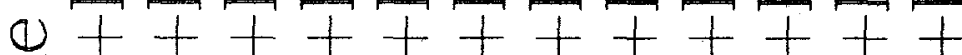

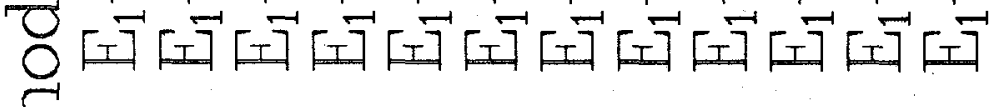




\section{A. Proof of Unbiasedness of Deconvolution Approach to Splitting}

Although it is probably obvious why the deconvolution approach produces an unbiased mean from the examples, a simple proof is given here for completeness. The proof shows the intuitive notion that sampling $m$ random walks and randomly discarding all but one is simply another way of sampling the original random walk. Before proceeding, note that the random number sequence completely determines the random walk so that showing that a random number sequence occurs with the same density in two cases shows that the corresponding random walks occur with the same density.

The proof proceeds in two steps. First, it is shown that splitting and randomly selecting one of the split branches results in the same density of random walks as the analog case. Second, the sampling of the tree is replaced by an expected value technique.

Consider any point in the random walk where an $m: 1$ split has occurred. Let $u(\mathbf{r})$ be the probability density function for the random sequence $\mathbf{r}$ that would be used subsequent to this point (in an analog calculation), and let the $m$ split branches use random sequences $\mathbf{r}_{\mathbf{i}}=\left(r_{i 1}, r_{i 2}, r_{i 3}, \cdots\right)(i=1, \cdots, m)$ drawn independently from $u\left(\mathbf{r}_{\mathbf{i}}\right)$. If the $i^{\text {th }}$ split branch is selected with probability $\frac{1}{m}$, then the probability $\left(p_{s}(\mathbf{r}) d \mathbf{r}\right.$ in the "split and sample" case) that the random sequence that would be used subsequent to this point is in $d \mathbf{r}$ about $\mathbf{r}$ is:

$$
p_{s}(\mathbf{r}) d \mathbf{r}=\sum_{k=1}^{m} \frac{1}{m} \int d \mathbf{r}_{\mathbf{1}} \cdots \int d \mathbf{r}_{\mathbf{k}} \cdots \int d \mathbf{r}_{\mathbf{m}}\left(u\left(\mathbf{r}_{\mathbf{1}}\right) \cdots u\left(\mathbf{r}_{\mathbf{k}}\right) \delta\left(\mathbf{r}_{\mathbf{k}}-\mathbf{r}\right) \cdots u\left(\mathbf{r}_{\mathbf{m}}\right)\right) d r
$$

The right side is the sum of the probabilities, over all possible ways, that the selected branch will use $\mathbf{r}$ in its random walk. Note that $\frac{1}{m}$ is the probability of selecting the $k^{t h}$ branch, and there is a sum over all possible choices for $k$. Further note that:

$$
\begin{gathered}
u\left(\mathbf{r}_{\mathbf{i}}\right) d \mathbf{r}_{\mathbf{i}}=\text { probability that } \mathbf{r}_{\mathbf{i}} \text { is in } d \mathbf{r}_{\mathbf{i}} \\
u\left(\mathbf{r}_{\mathbf{k}}\right) d \mathbf{r}_{\mathbf{k}} \delta\left(\mathbf{r}_{\mathbf{k}}-\mathbf{r}\right) d \mathbf{r}=\text { probability that } \mathbf{r}_{\mathbf{k}} \text { is in } d \mathbf{r}_{\mathbf{k}} \text { and in } d \mathbf{r}
\end{gathered}
$$

The integrations sum over all possible values of the $\mathbf{r}_{\mathbf{i}}$. Performing the indicated integrations:

$$
\begin{gathered}
\int u\left(\mathbf{r}_{\mathbf{i}}\right) d \mathbf{r}_{\mathbf{i}}=1 \\
\int u\left(\mathbf{r}_{\mathbf{k}}\right) \delta\left(\mathbf{r}_{\mathbf{k}}-\mathbf{r}\right) d \mathbf{r}_{\mathbf{k}} d \mathbf{r}=u(\mathbf{r}) d \mathbf{r}
\end{gathered}
$$

so that,

$$
p_{s}(\mathbf{r}) d \mathbf{r}=u(\mathbf{r}) d \mathbf{r}
$$


Thus, the distribution of "split and sample" random walks is identical to the distribution of analog random walks.

Rather than sample each of the split branches to determine which tree occurs, one can look at all possible trees and tally the expected tally. That is, suppose that tree $i$ occurs with probability $q_{i}$ and produces a tally $t_{i j}$ in bin $j$. The expected tally in bin $j$ over all possible trees is:

$$
<T_{j}>=\sum_{i} q_{i} t_{i j} .
$$

The sampling is now replaced by tallying $\left\langle T_{j}>\right.$ with probability one and the mean is preserved.

\section{B. Deconvolution of a Typical Tree}

Note that when there is a physical split, the number of choices is the product of the number of choices on each of the physical branches. Thus, in a complicated tree with many physical and variance reduction splits the number of choices may become overwhelmingly large. A solution to this problem will be proposed later. For now, a more typical random walk tree is discussed to show that the number of choices usually does not become overwhelming.

The tree of Fig. 2 was done with an arbitrary set of $E_{k}$ to demonstrate the deconvolution technique. In most cases that require splitting, most of the $E_{k}$ are zero. Furthermore, the nonzero $E_{k}$ tend to be at the bottom of the trees because photons depositing energy in the detector are usually the photons that have undergone the most splitting. The tree of Fig. 13 is not an atypical example of such trees. Note that most branches do not have an energy deposited listed adjacent to them; there is zero energy deposited when no energy is listed.

The analysis proceeds from the highest numbered node as in the previous case. Figures 13-30 show the deconvolution for this case. In the previous case, node 1 was a physical split and resulted in the product of the choices from the left and right branches. Unlike the previous case (Fig. 2), there is no product of choices that can cause relatively large numbers of choices. Here, for example, node 1 is a variance reduction node and thus the number of choices at node 1 is equal to the sum of the choices from the left and right branches. 


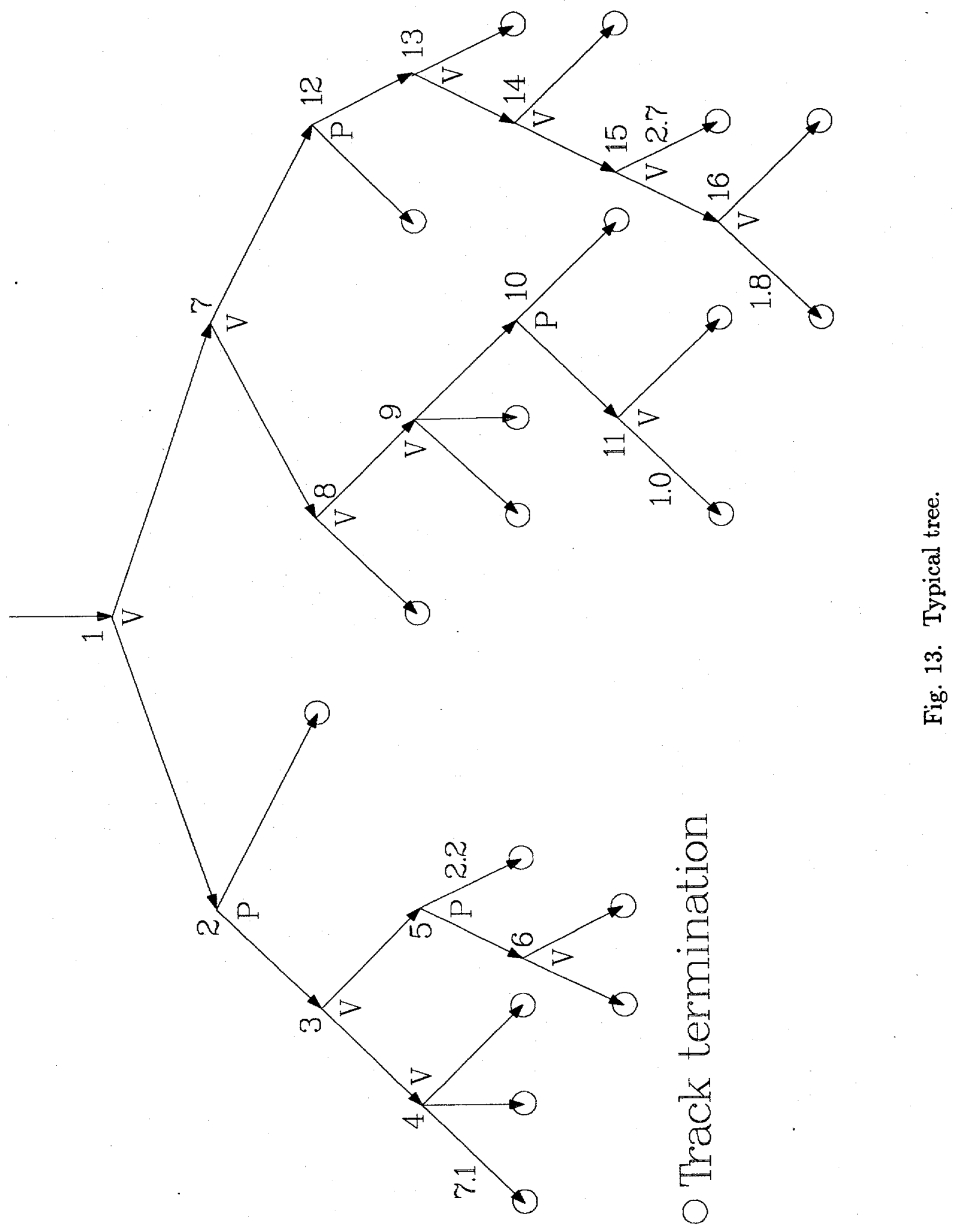




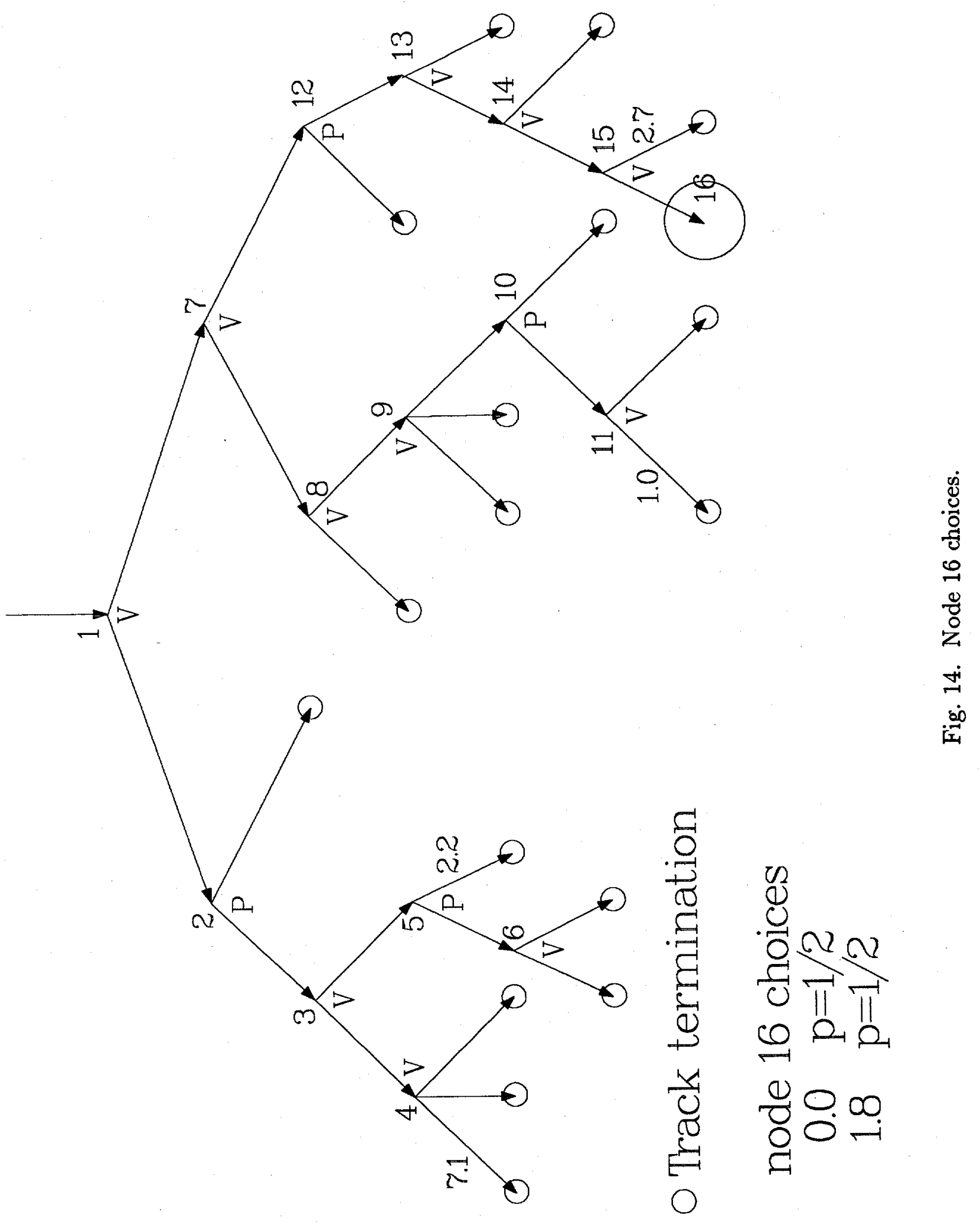




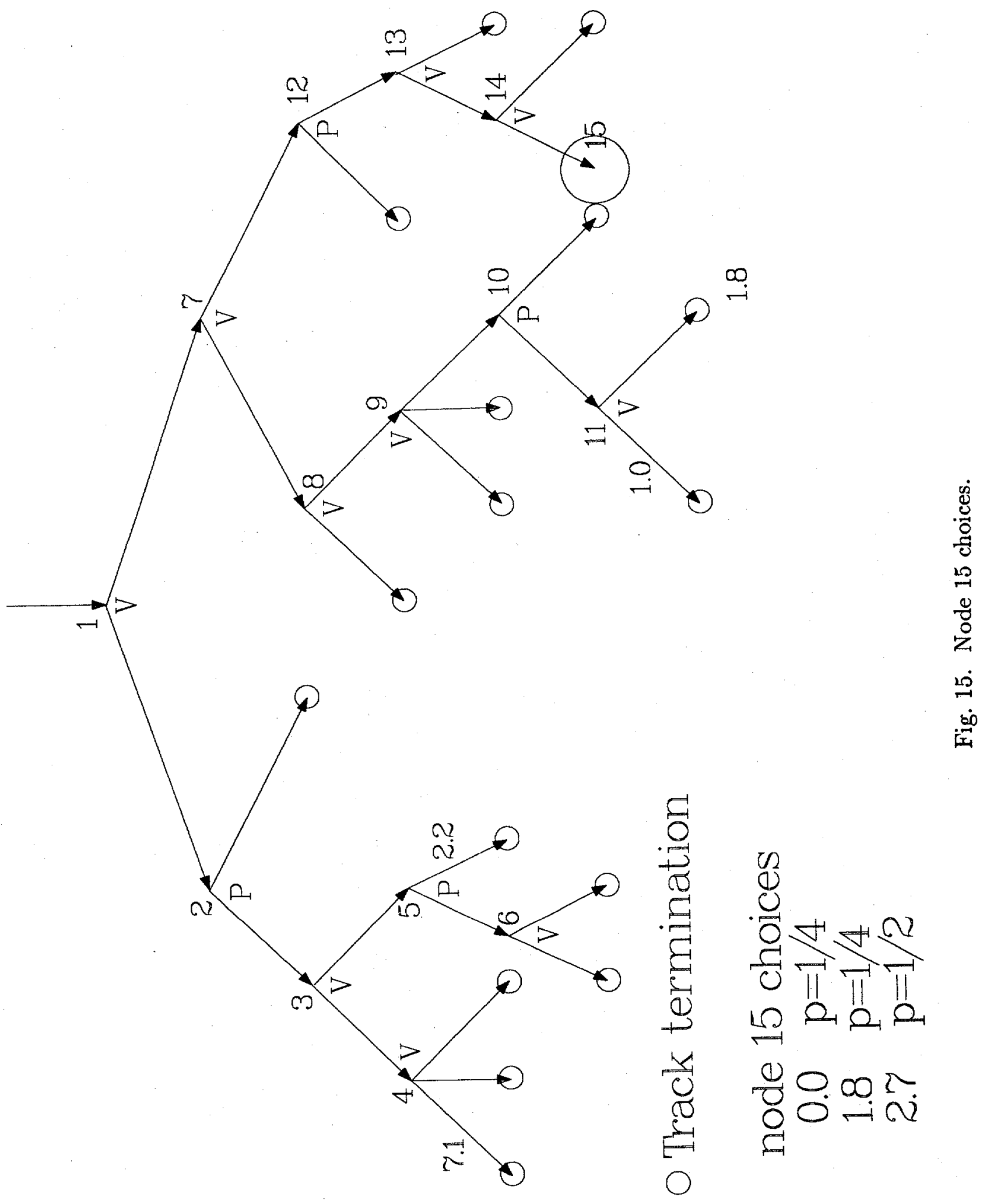




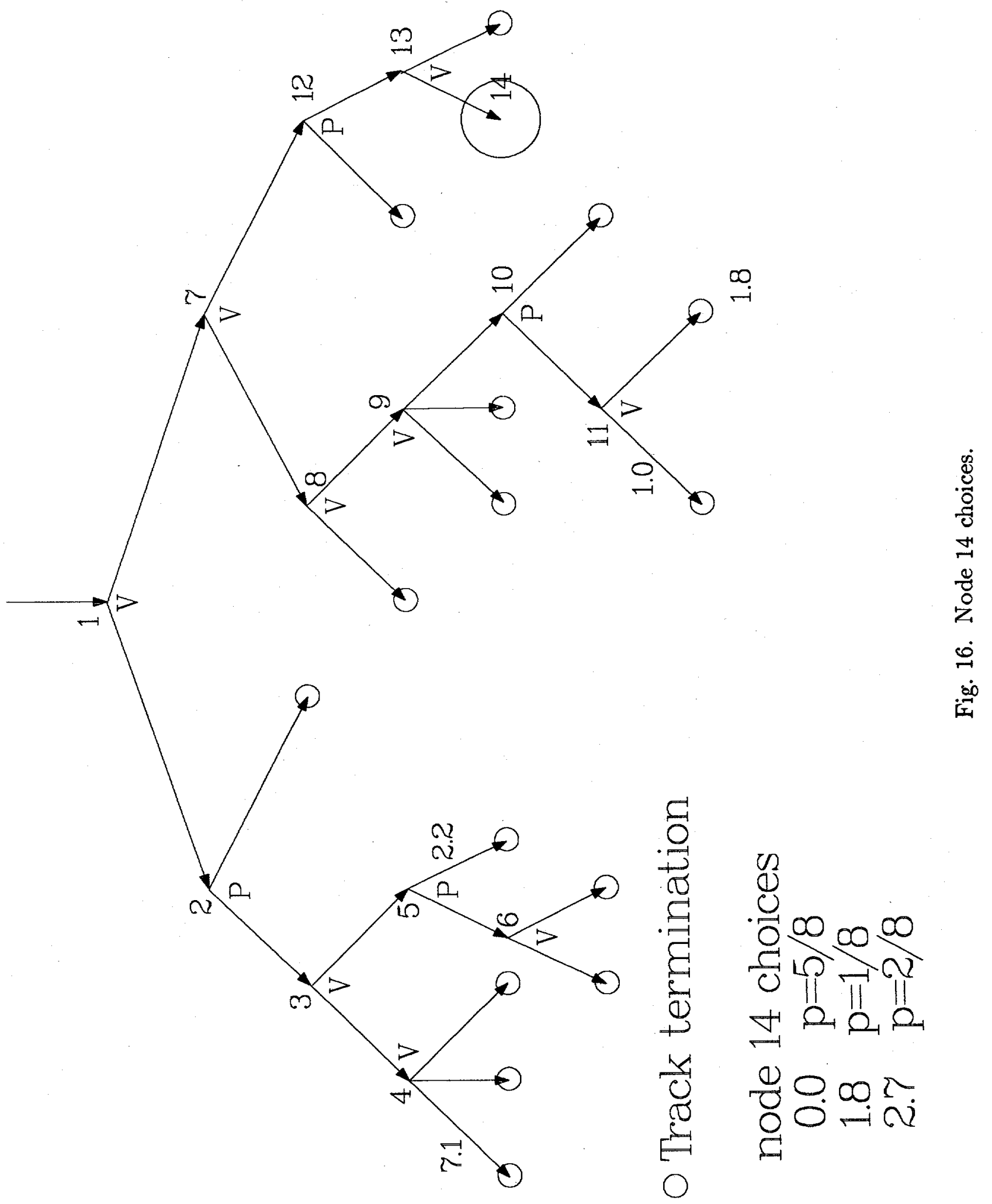




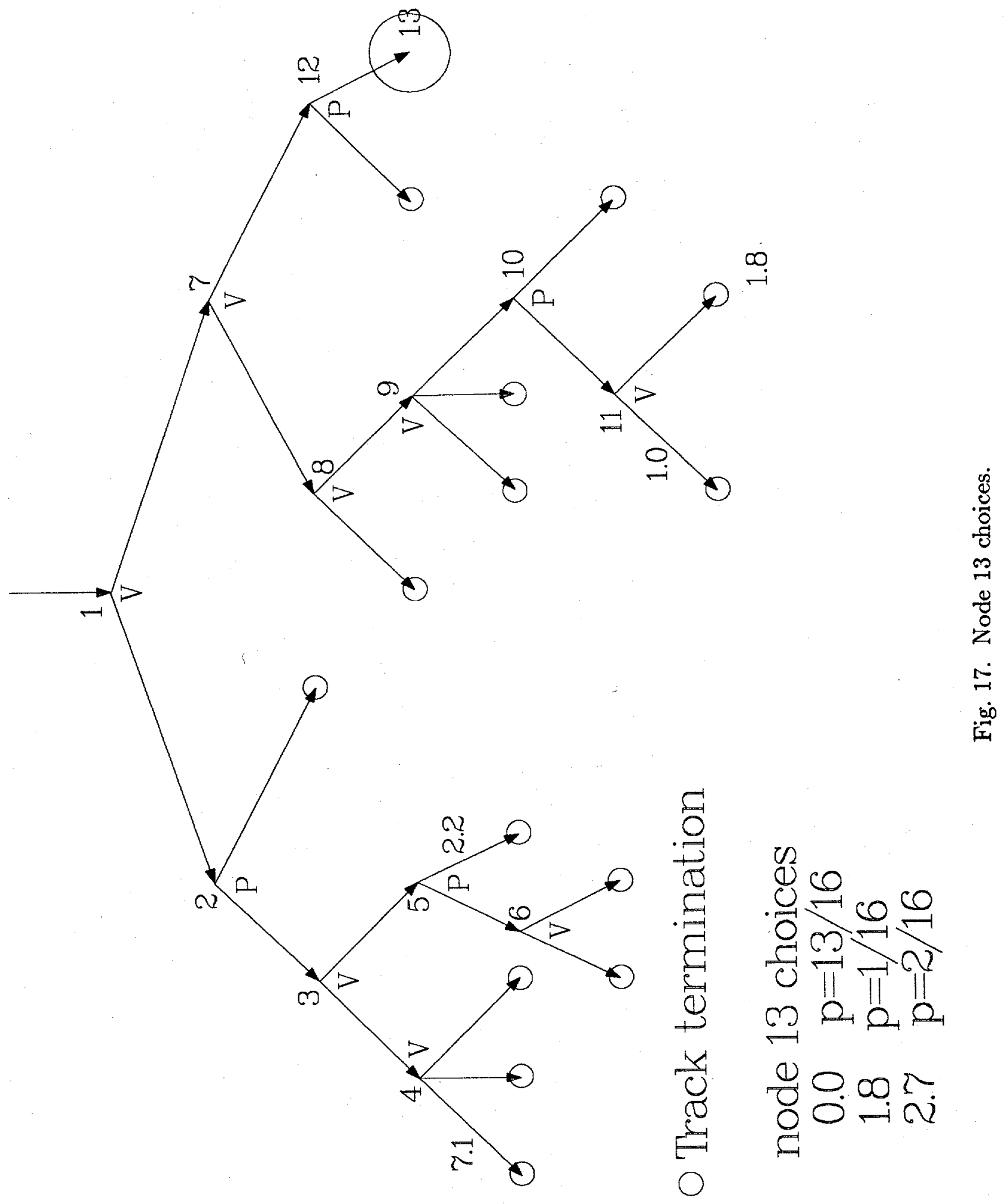




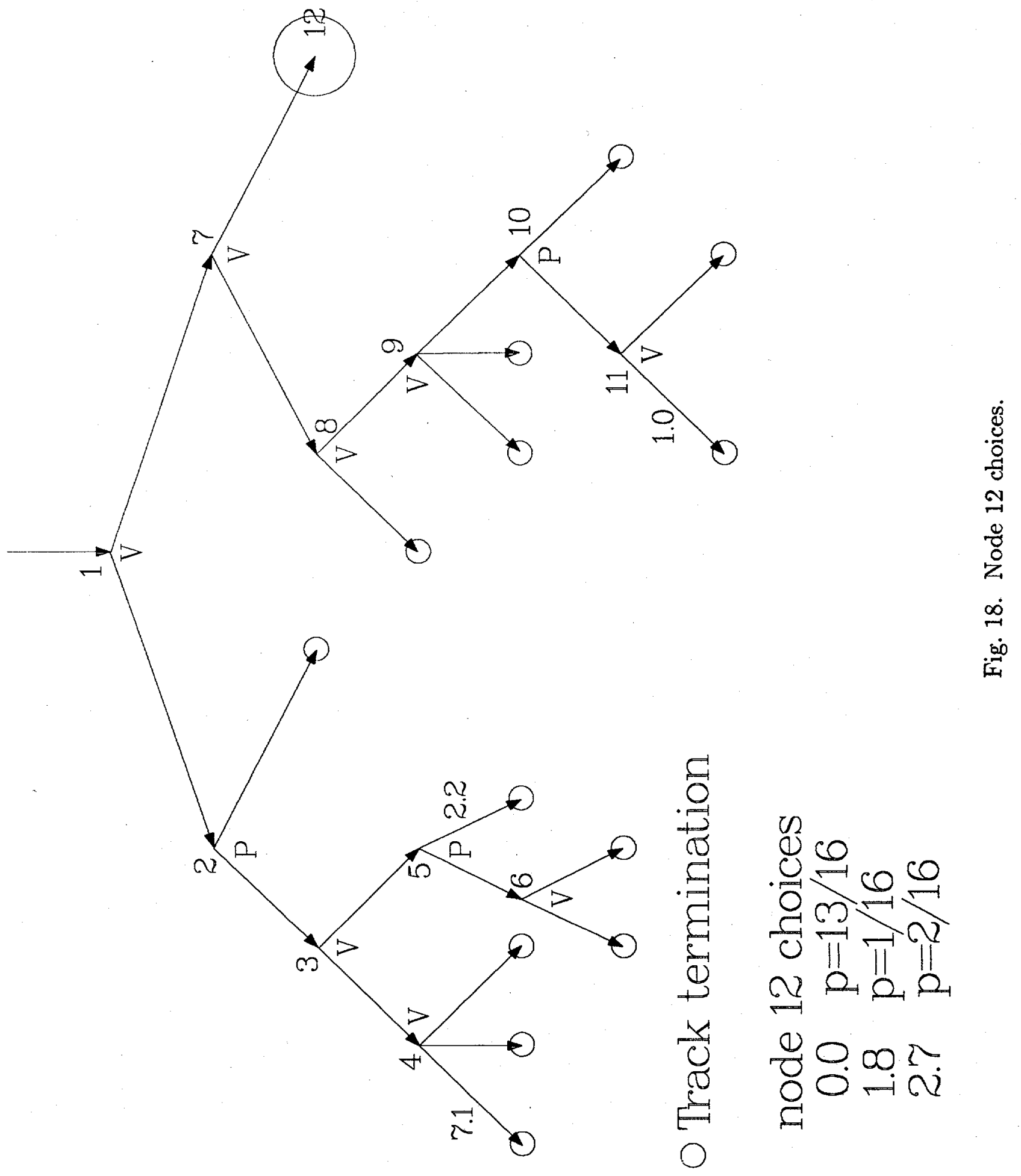




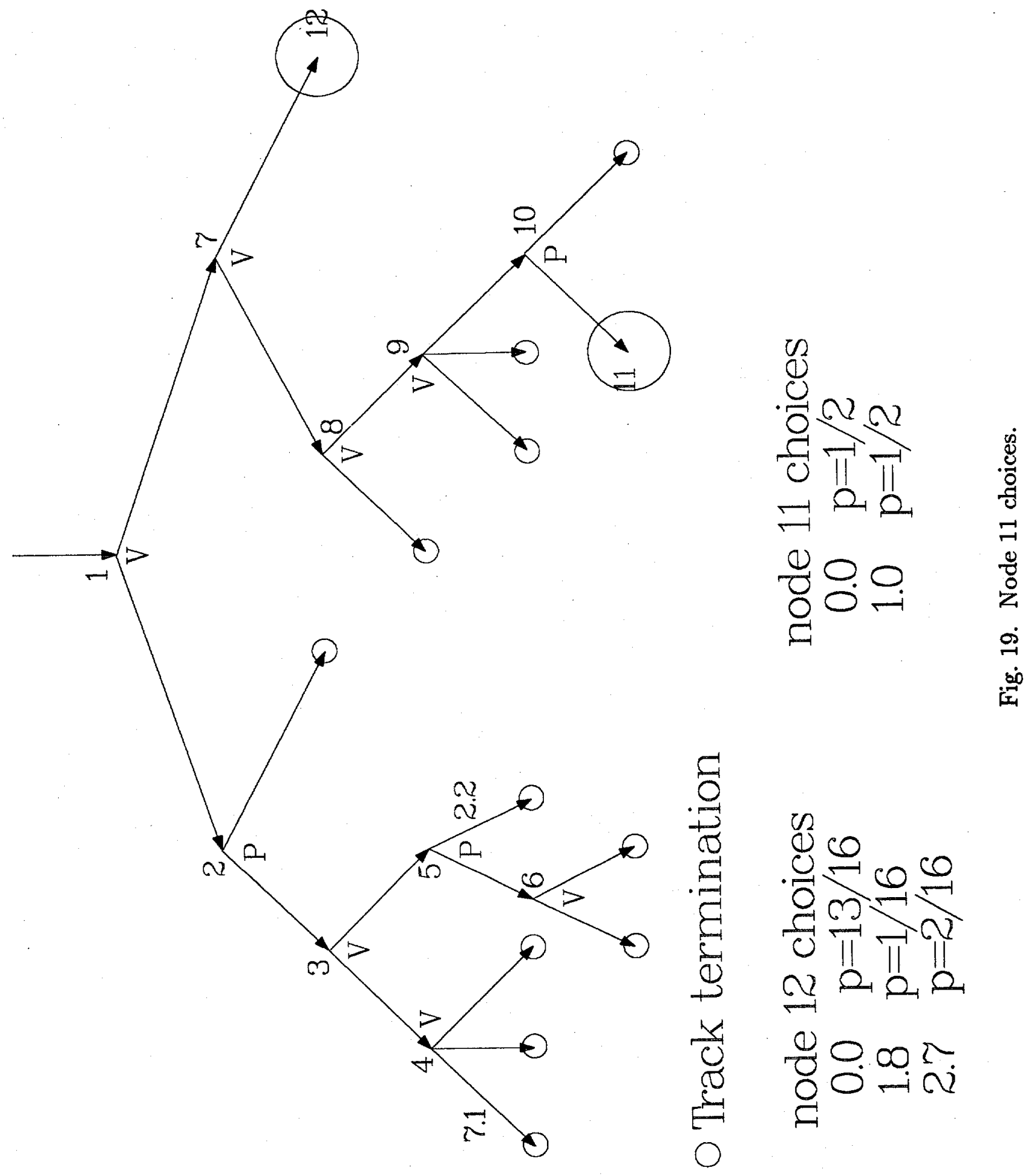




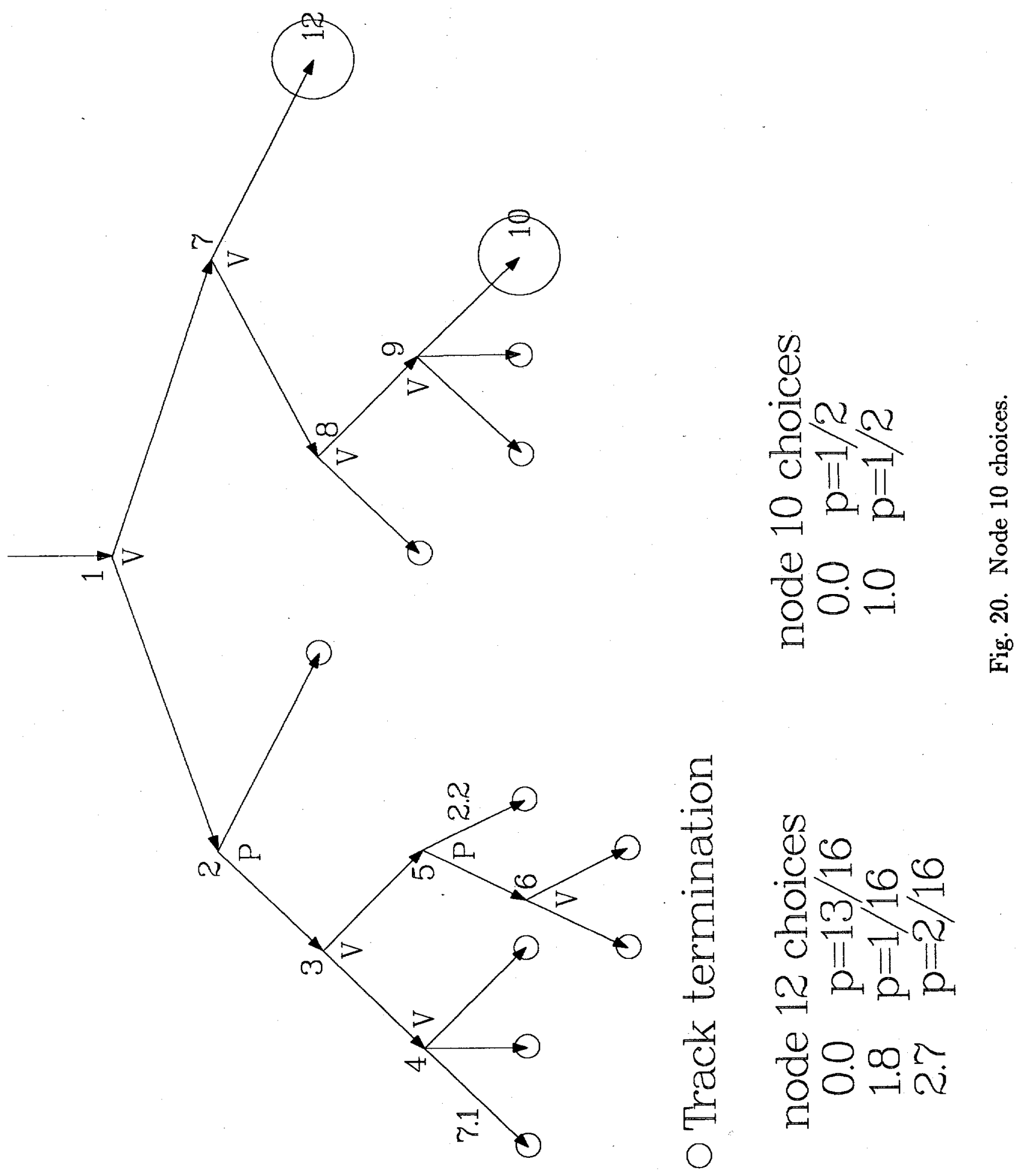




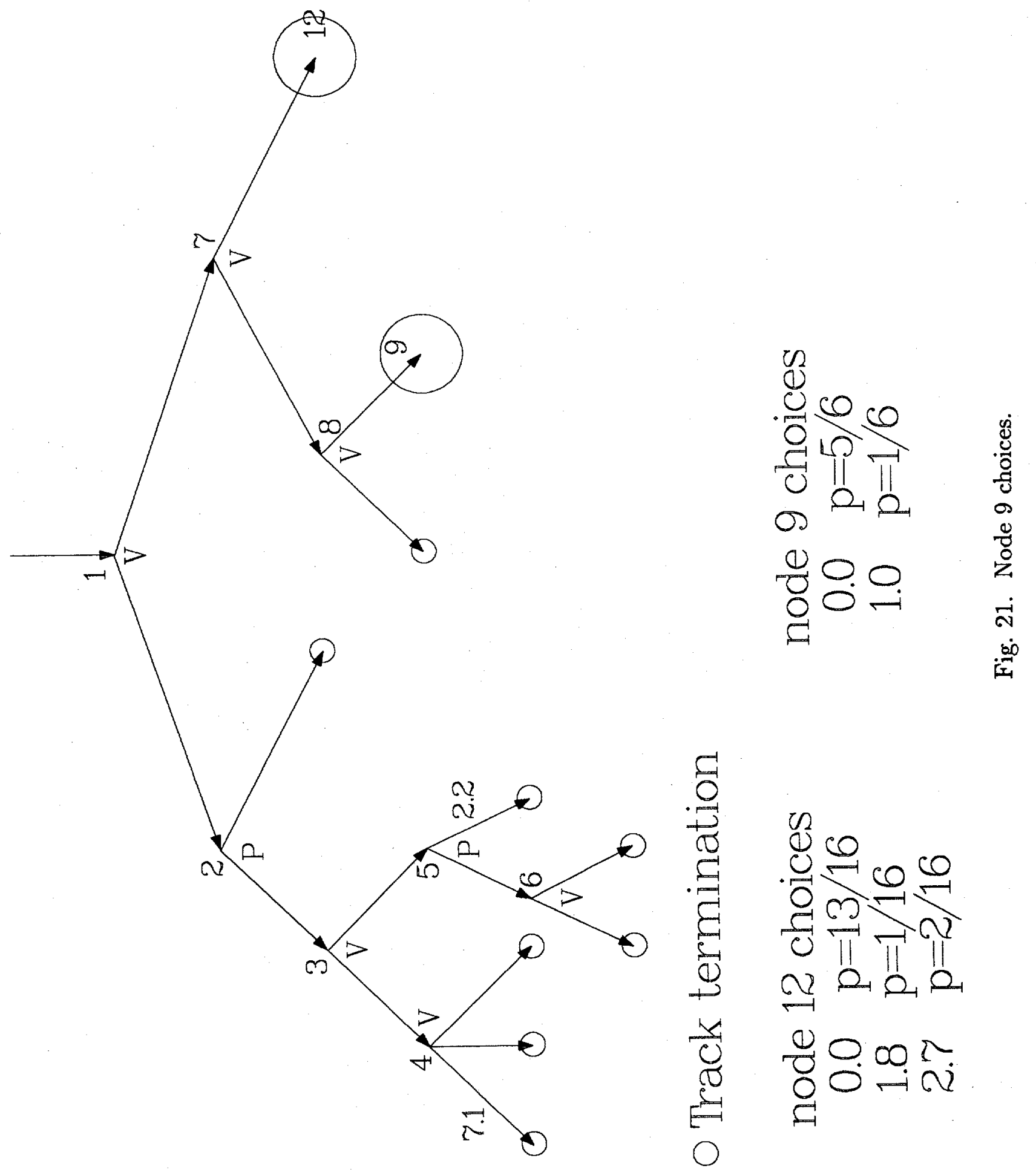




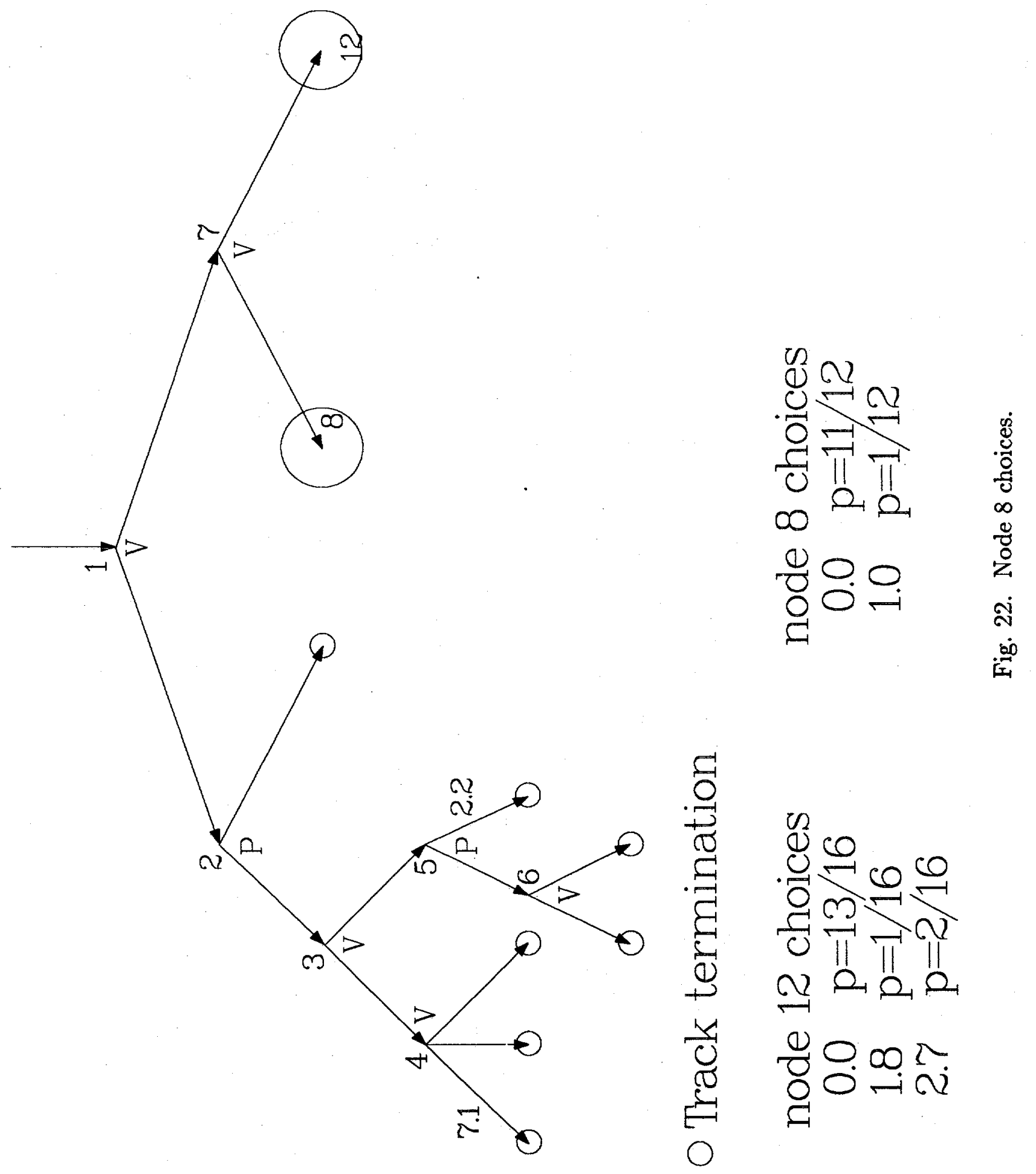




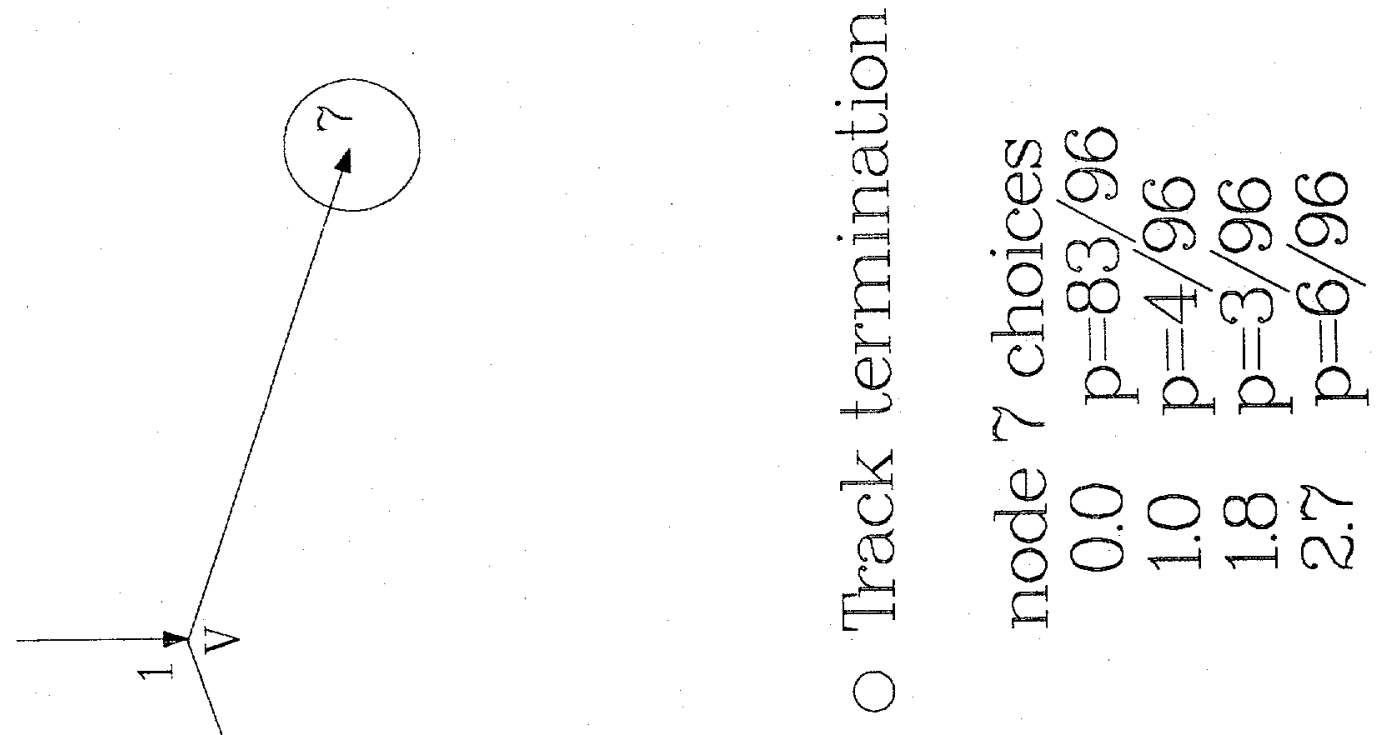

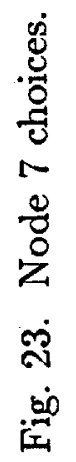




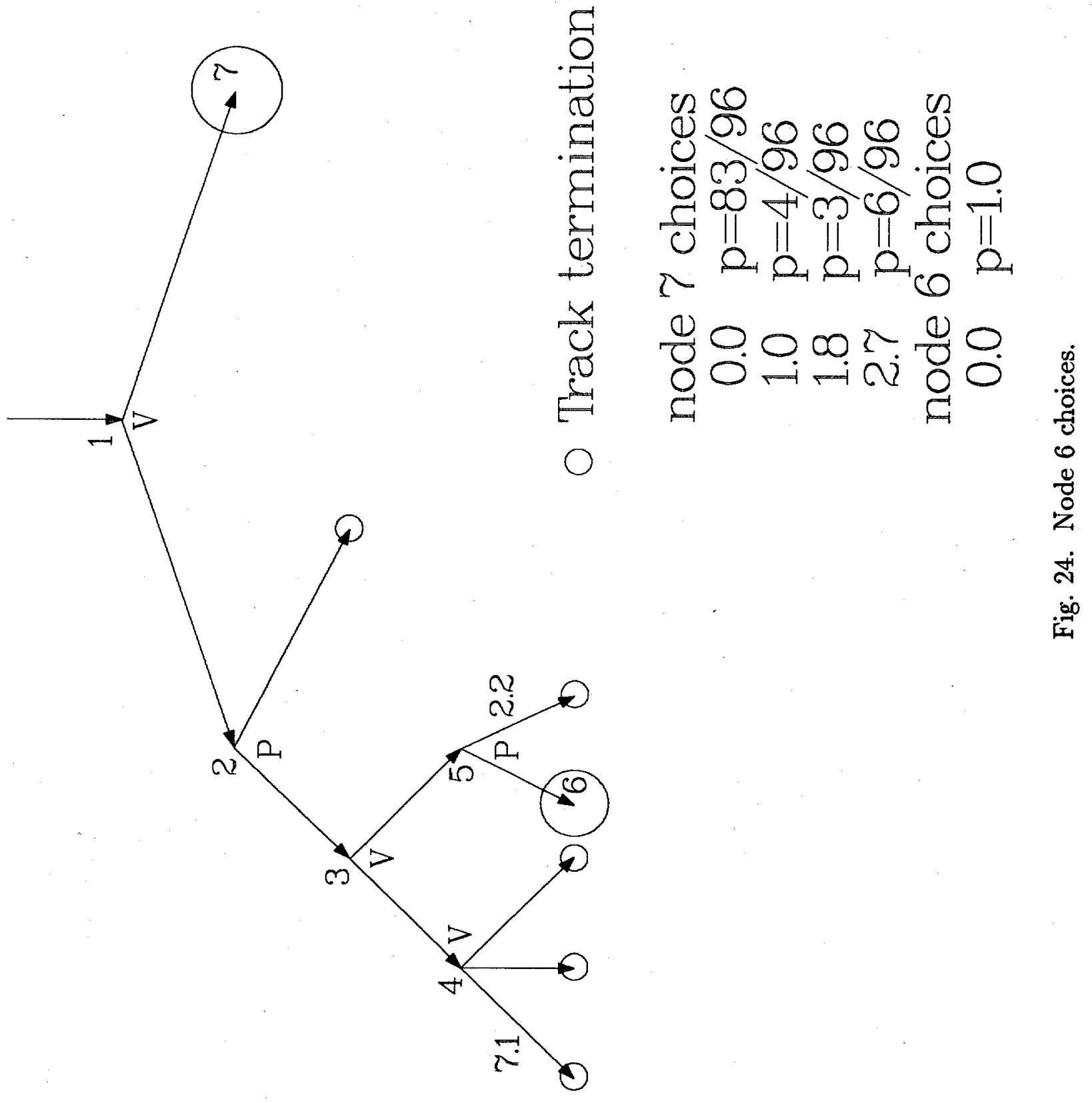




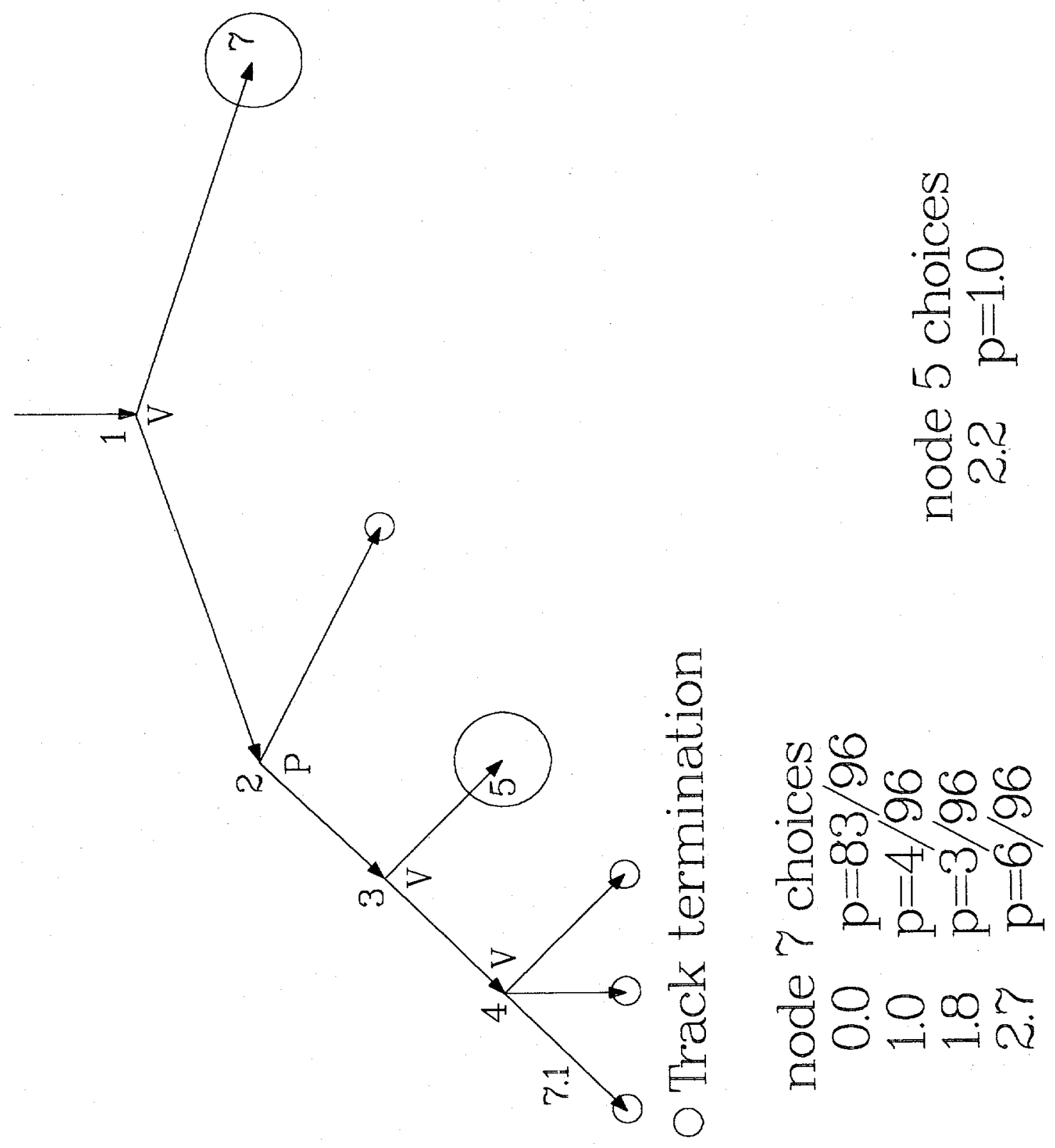



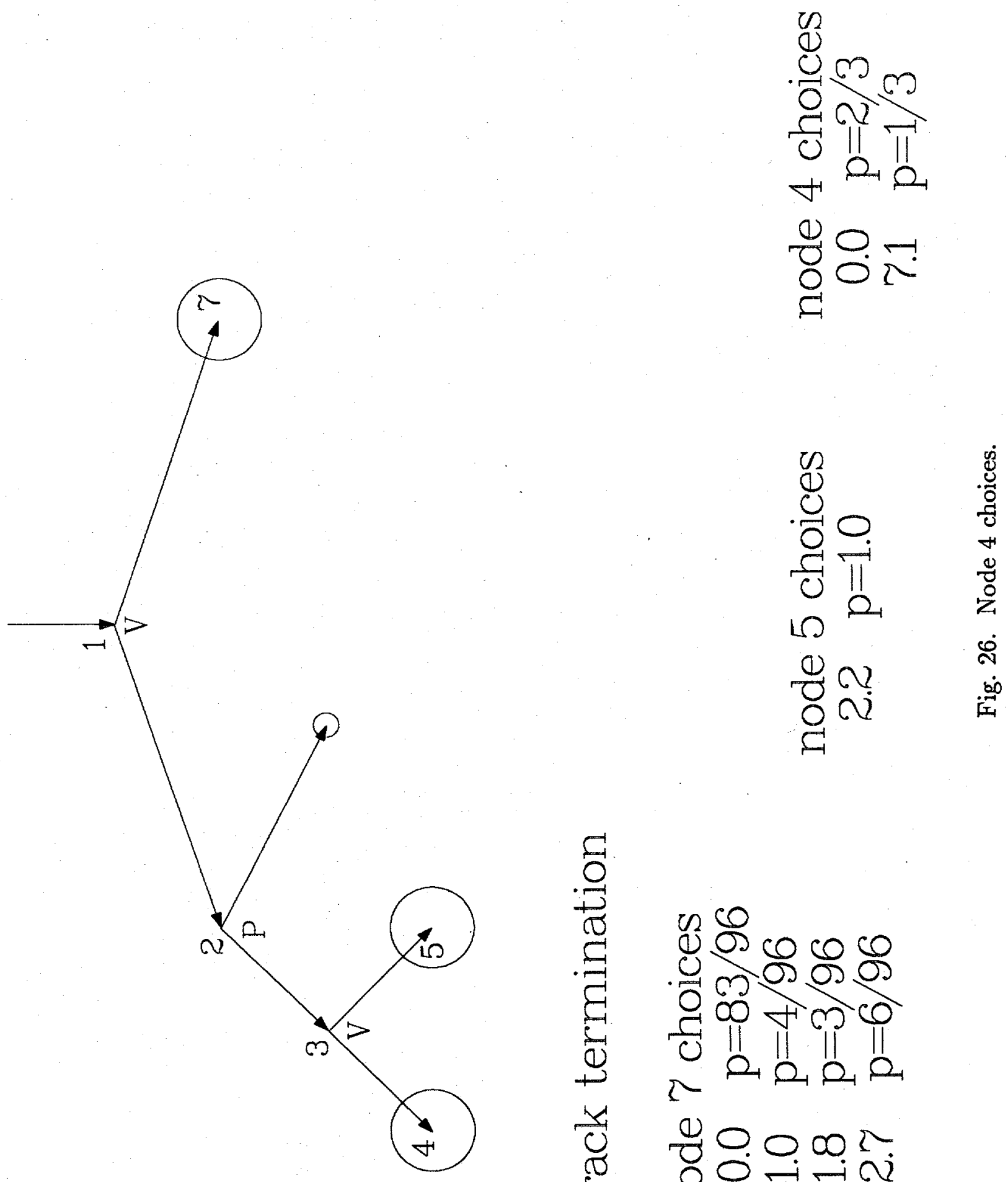

\&े

空

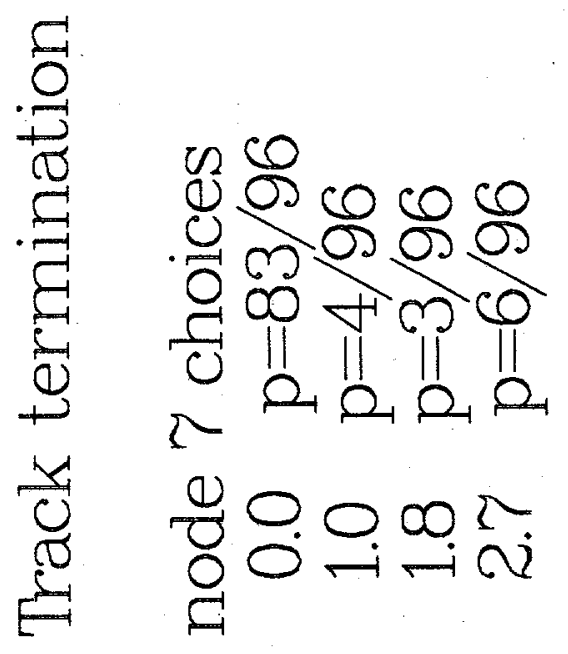



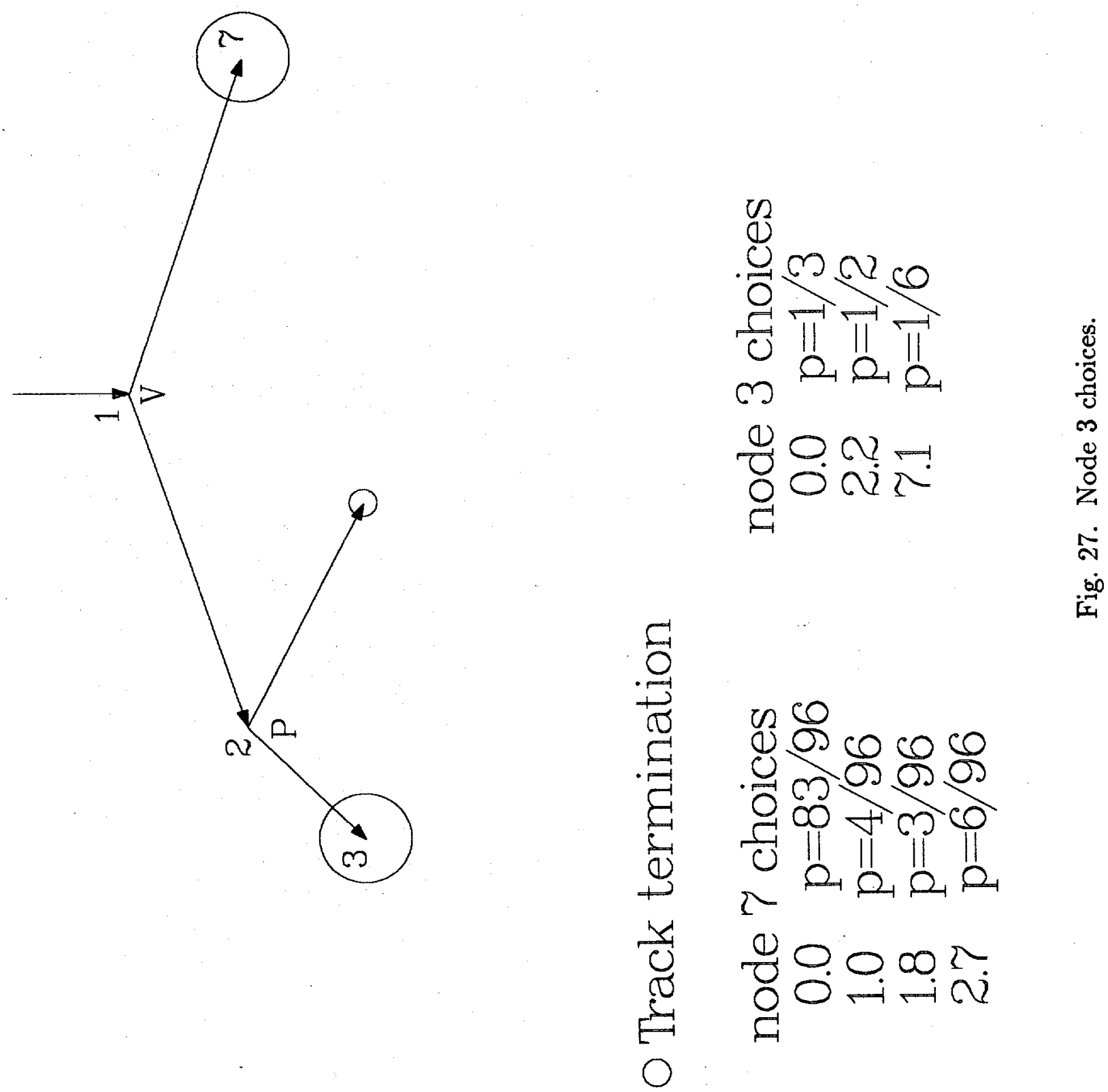

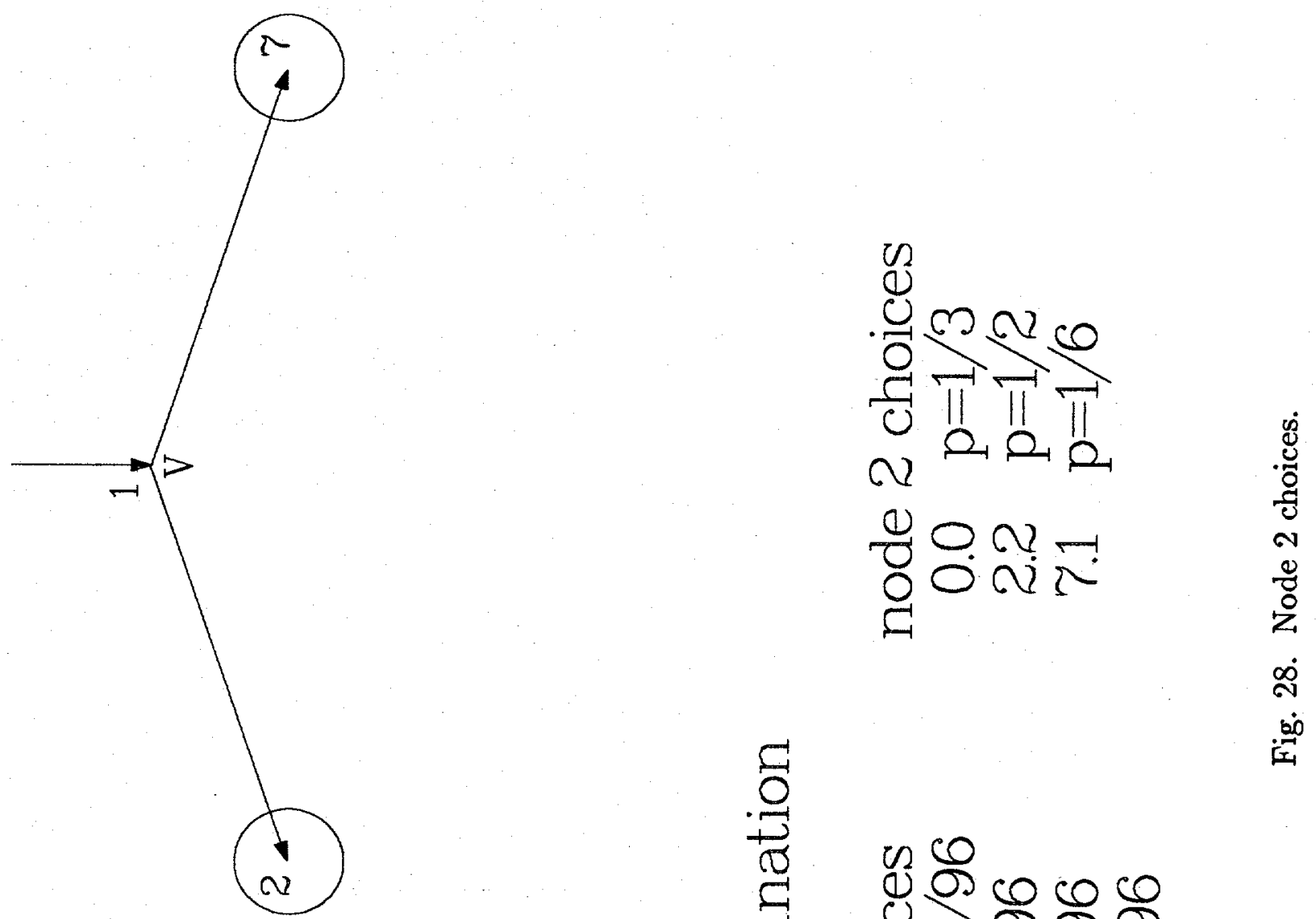

.0.0

ये 

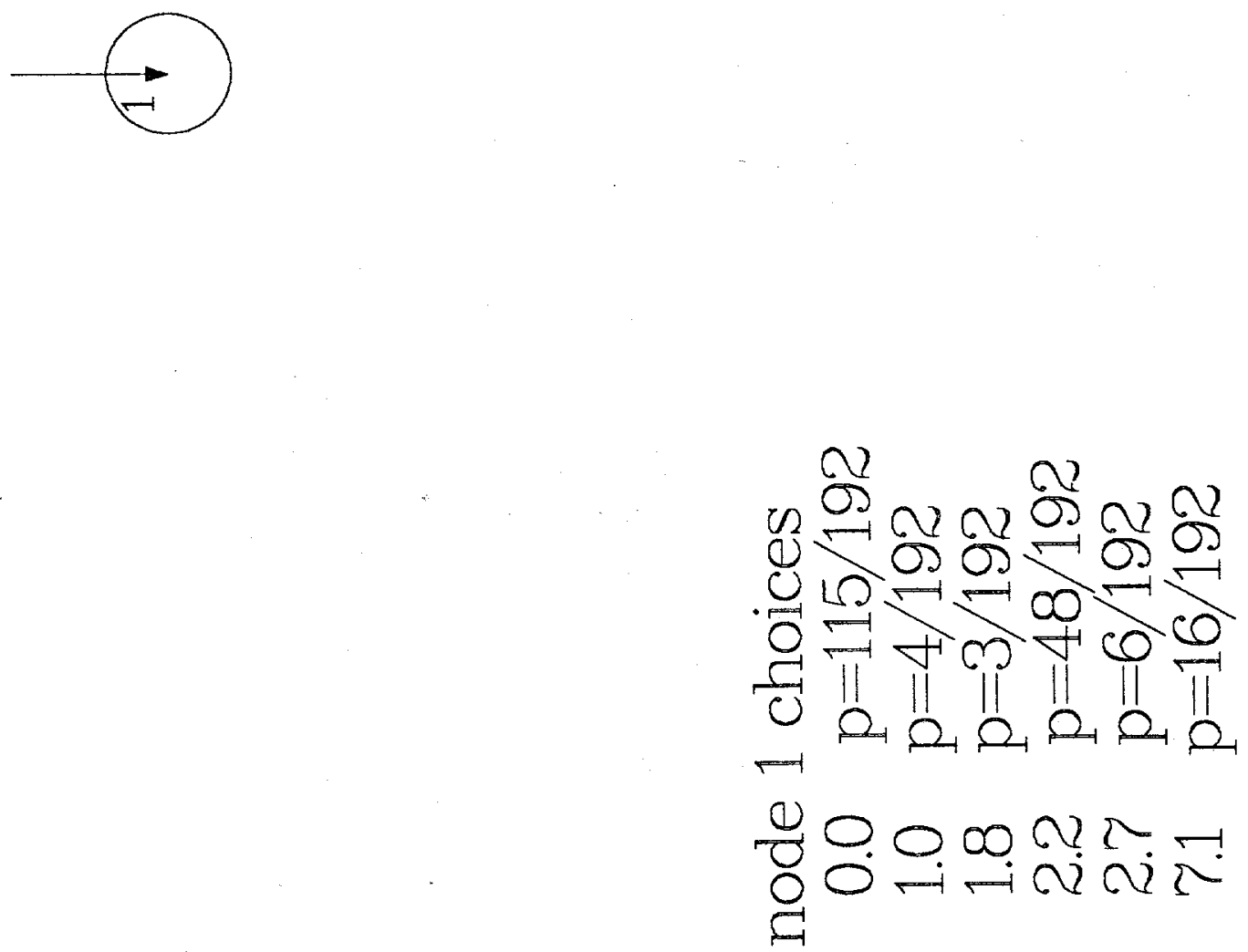

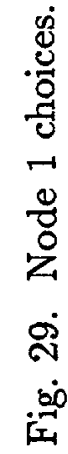




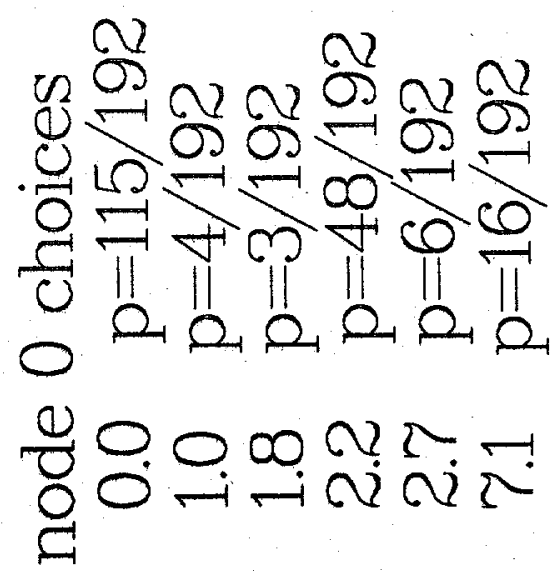




\section{Thinning Large Trees}

Although rare (for pulse height tallies), it is possible in a complicated tree with many physical and variance reduction splits that the number of choices may become overwhelmingly large. For example, if there is a three-branch physical split below the current node and if each of the branches has 100 possible choices for energy deposited, then there will be a million choices at the current physical node. In these cases the choices can be "thinned" in a number of possible ways.

As an example of one possible thinning procedure, consider the deconvolution in Fig. 10. Suppose that one decides that there will be no more than 10 choices at each node. Note that the deconvolution provides $3 \cdot 4=12$ choices (Fig. 11). Suppose that before deconvoluting Fig. 10, a random number decides which of the first two choices of node 2 will survive with doubled weight and which will die. For example, with probability $\frac{1}{2}$ Fig. 10 is converted into Fig. 31 and with probability $\frac{1}{2}$ Fig. 10 is converted into Fig. 32 . The deconvolution process proceeds as before except that the number of choices at node 1 will now be $3 \cdot 3=9$, whether the conversion is into Fig. 31 or Fig. 32. The expected choices at node 1 will be the same as without the thinning. All the choices that involve neither $E_{3}+E_{4}$ nor $E_{3}+E_{5}$ are unaffected by the thinning, and the choices associated with $E_{3}+E_{4}$ will occur only half the time but with twice the weight $(\mathrm{p})$ and similarly for $E_{3}+E_{5}$.

\section{Summary of Splitting Using the Deconvolution Approach}

Non-Boltzmann tallies (such as pulse height tallies) can be correctly done using the standard track splitting game typically applied to standard tallies like flux or current. The primary advantage of this approach is that it allows non-Boltzmann tallies to be obtained with the same tracking procedure that is currently applied to tallies like flux and current. The approach permits standard Monte Carlo codes to get non-Boltzmann tallies using the same particle tracking algorithms as employed for tallies such as flux. There are two disadvantages to this approach, one practical and one philosophical. As a practical matter, there is significant extra bookkeeping required, and this bookkeeping is likely to get more onerous as other standard trackbased variance reduction techniques are used at the same time as splitting. The deconvolution approach is also unappealing from a philosophical viewpoint because the physics is buried in the deconvolution rather than being explicitly expressed in the transport algorithm. Effective use of variance reduction techniques is usually

guided by the Monte Carlo practitioner's intuitive understanding of the natural transport process and the information supplied by short scoping calculations with the Monte Carlo code. The more divorced the natural transport process becomes from the Monte Carlo algorithm, the more difficult it becomes for the practitioner to use his intuition and to interpret the information supplied by short scoping calculations. 

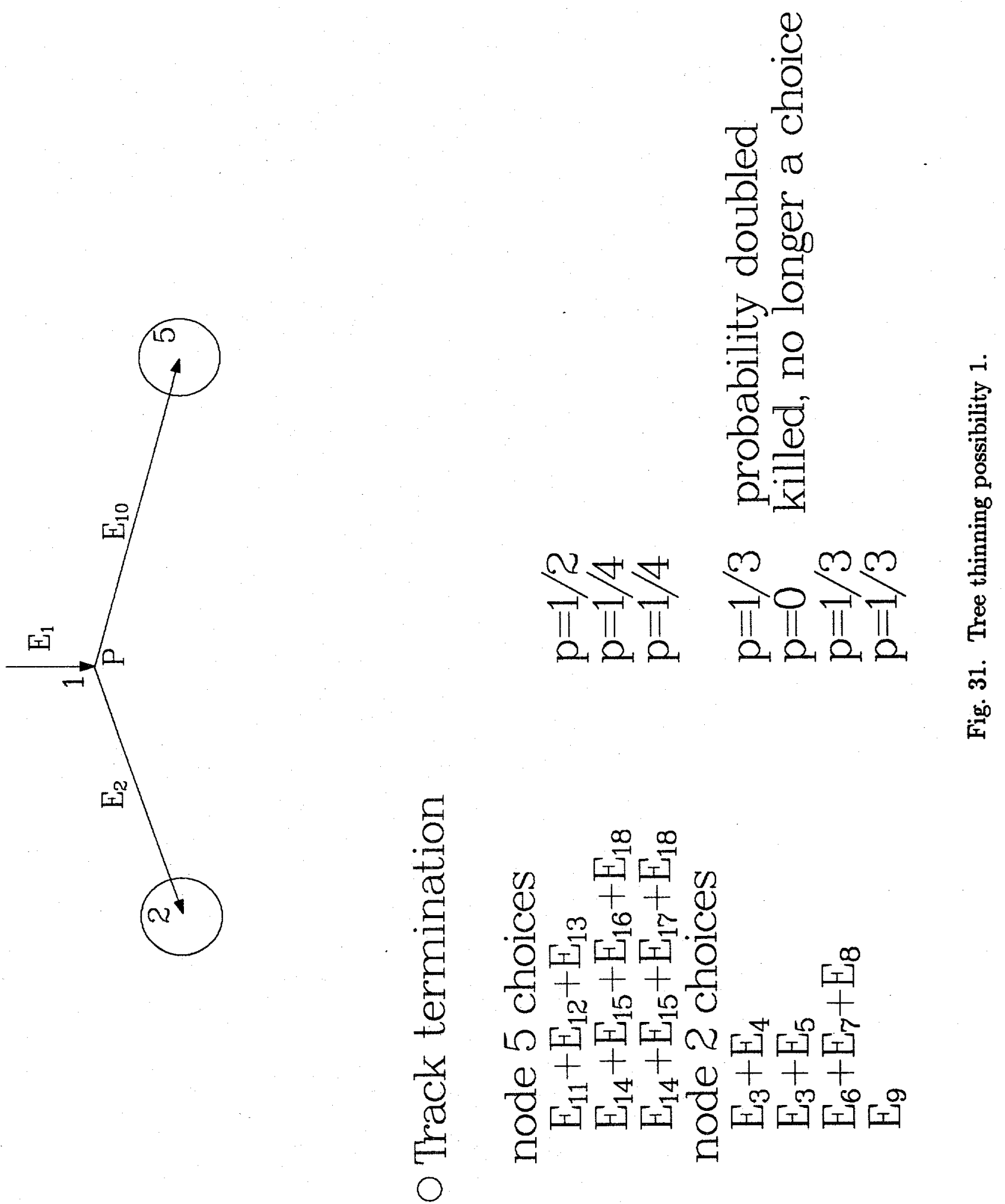

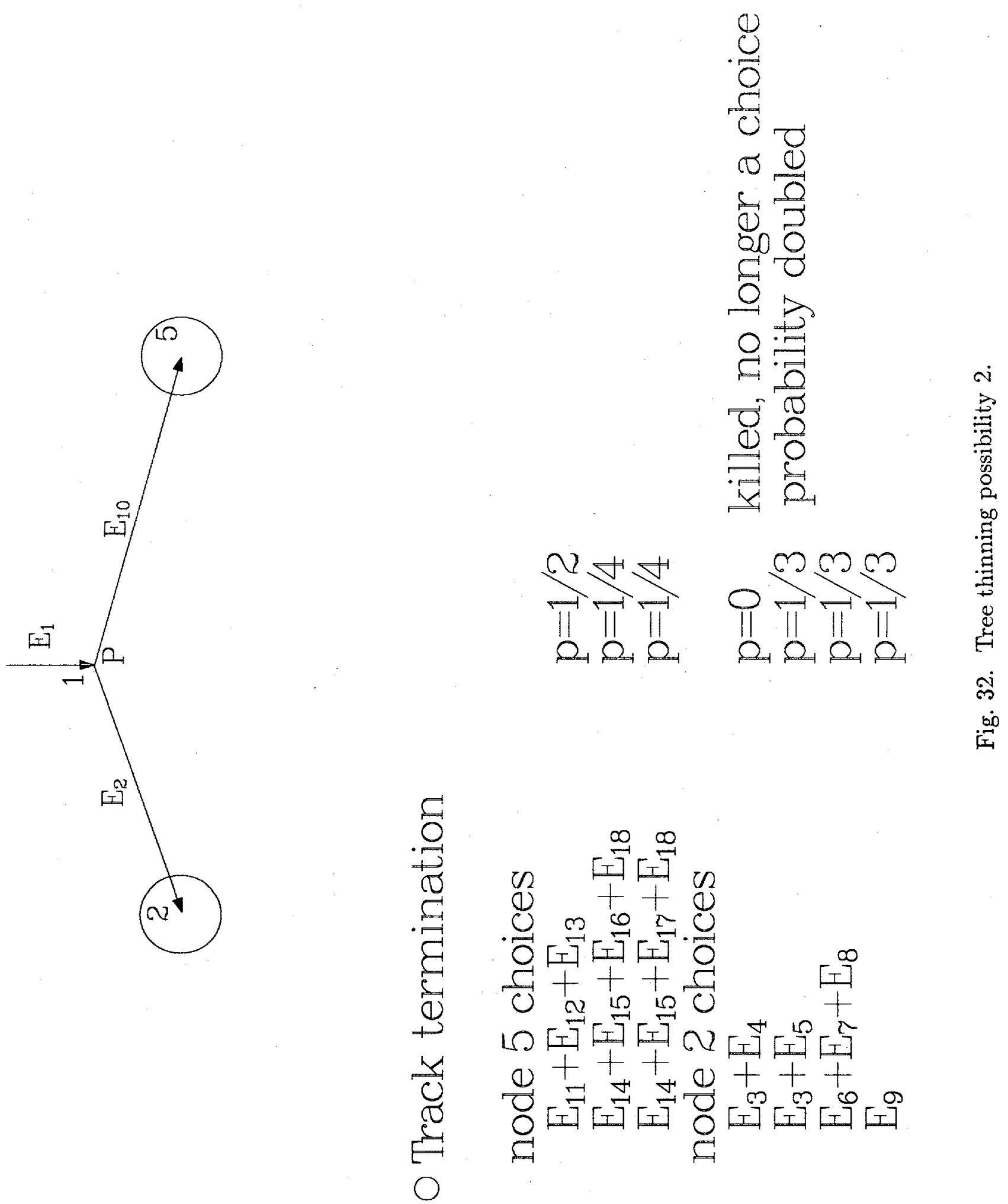

(2) $\quad \stackrel{\infty}{+\frac{\infty}{+}\left[\frac{9}{7}\right.}[2$

(1) $+1+0$

-

o + in $+\underset{0}{0}$

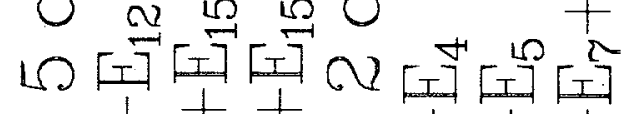

Q $0+++\infty++$

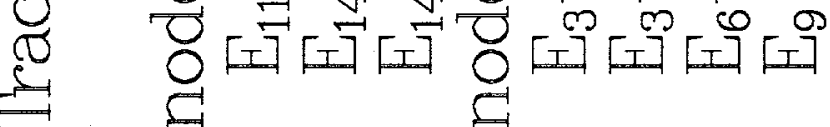

O 


\section{NON-BOLTZMANN TALLIES WITH THE CORRECTED SIN- GLE PARTICLE APPROACH}

The corrected single particle approach treats the tracks as if they were all independent entities and tallies the tracks independently without regard to their relationships to other tracks. That is, the approach treats the tracks as if the tally were a standard Boltzmann-type tally and then later corrects for this mistreatment. The approach can be motivated by inspecting the tallies made with the deconvolution approach on the random walk of Fig. 13. The tallies from the final deconvolution are shown in Fig. 30. Note from Fig. 13 that these are exactly the same tallies that would have been made if each track were tallied independently from all other tracks. That is, the energy deposited from one track is not added to the energy deposited from any other tracks to determine the energy bin in which to tally. This procedure would be the normal way to post Boltzmann tallies, treating each track as an independent entity with its own weight. For example, if the track on the left branch of node 16 were treated as a separate entity, its weight would be $1 / 64=3 / 192$ because of the six 2:1 splits tracing its way back to the source. The total energy deposited along its path back to the source is 1.8 , and thus the Boltzmann tally would be $1 / 64$ in the energy bin containing 1.8. If the deconvolution approach usually produces the same tally as the standard Boltzmann tally technique, then much time will be wasted doing the deconvolution only to find out that the tallies were identical. Considerable time might be saved by doing the full deconvolution on a fraction of the histories, say $10 \%$, and doing the standard Boltzmann tally on $90 \%$ of the histories. The corrected tally would then be the standard Boltzmann tally on $100 \%$ of the histories plus 10 times the difference between the deconvolution approach and the standard Boltzmann approach on the $10 \%$ that are tallied both ways. A proof that this method is unbiased will be presented after a specific example is given to clarify the method.

To illustrate how the difference between the deconvolution approach and the standard Boltzmann approach is obtained, consider the random walk of Fig. 2 and its deconvolution, Fig. 12. The standard Boltzmann approach (which is not correct here) would treat the tracks as independent entities and make no allowance for whether a split is a variance reduction split or a physical split. The total energy deposited would be the sum of all the energy deposited along the traceback route to the source. For example, in Fig. 2 starting from left to right the Boltzmann approach would score:

$$
\begin{aligned}
& E_{4}+E_{3}+E_{2}+E_{1} \quad \text { with tally }=\frac{1}{6} \\
& E_{5}+E_{3}+E_{2}+E_{1} \quad \text { with tally }=\frac{1}{6} \\
& E_{7}+E_{6}+E_{2}+E_{1} \quad \text { with tally }=\frac{1}{3}
\end{aligned}
$$




$$
\begin{array}{rr}
E_{8}+E_{6}+E_{2}+E_{1} \quad \text { with tally }=\frac{1}{3} \\
E_{9}+E_{2}+E_{1} \quad \text { with tally }=\frac{1}{3} \\
E_{12}+E_{11}+E_{10}+E_{1} \quad \text { with tally }=\frac{1}{2} \\
E_{13}+E_{11}+E_{10}+E_{1} \quad \text { with tally }=\frac{1}{2} \\
E_{16}+E_{15}+E_{14}+E_{10}+E_{1} \quad \text { with tally }=\frac{1}{4} \\
E_{17}+E_{15}+E_{14}+E_{10}+E_{1} \quad \text { with tally }=\frac{1}{4} \\
E_{18}+E_{14}+E_{10}+E_{1} \quad \text { with tally }=\frac{1}{2}
\end{array}
$$

Now consider the typical case in which most of the $E_{i}$ are zero. These figures are redrawn here (Figs. 33 and 34 ) with only the nonzero $E_{i}$ listed. The nonzero scores from the above (Boltzmann approach) equations are:

$$
\begin{aligned}
E_{3}+E_{2} & \text { with tally }=\frac{1}{6} \\
E_{3}+E_{2} & \text { with tally }=\frac{1}{6} \\
E_{7}+E_{6}+E_{2} & \text { with tally }=\frac{1}{3} \\
E_{8}+E_{6}+E_{2} & \text { with tally }=\frac{1}{3} \\
E_{9}+E_{2} & \text { with tally }=\frac{1}{3}
\end{aligned}
$$

The general deconvolution tallies are given in Fig. 12 and the specific case (with the zero $E_{i}$ ) of Fig. 34 is derived from it by explicitly inserting 0's for the $E_{i}$ that are zero. The bottom of Fig. 34 is a condensation (of the top) into identical scores. That is, for the deconvolution approach:

$$
\begin{array}{r}
E_{2}+E_{3} \quad \text { with tally }=\frac{1}{3} \\
E_{2}+E_{6}+E_{7}+E_{8} \quad \text { with tally }=\frac{1}{3}
\end{array}
$$




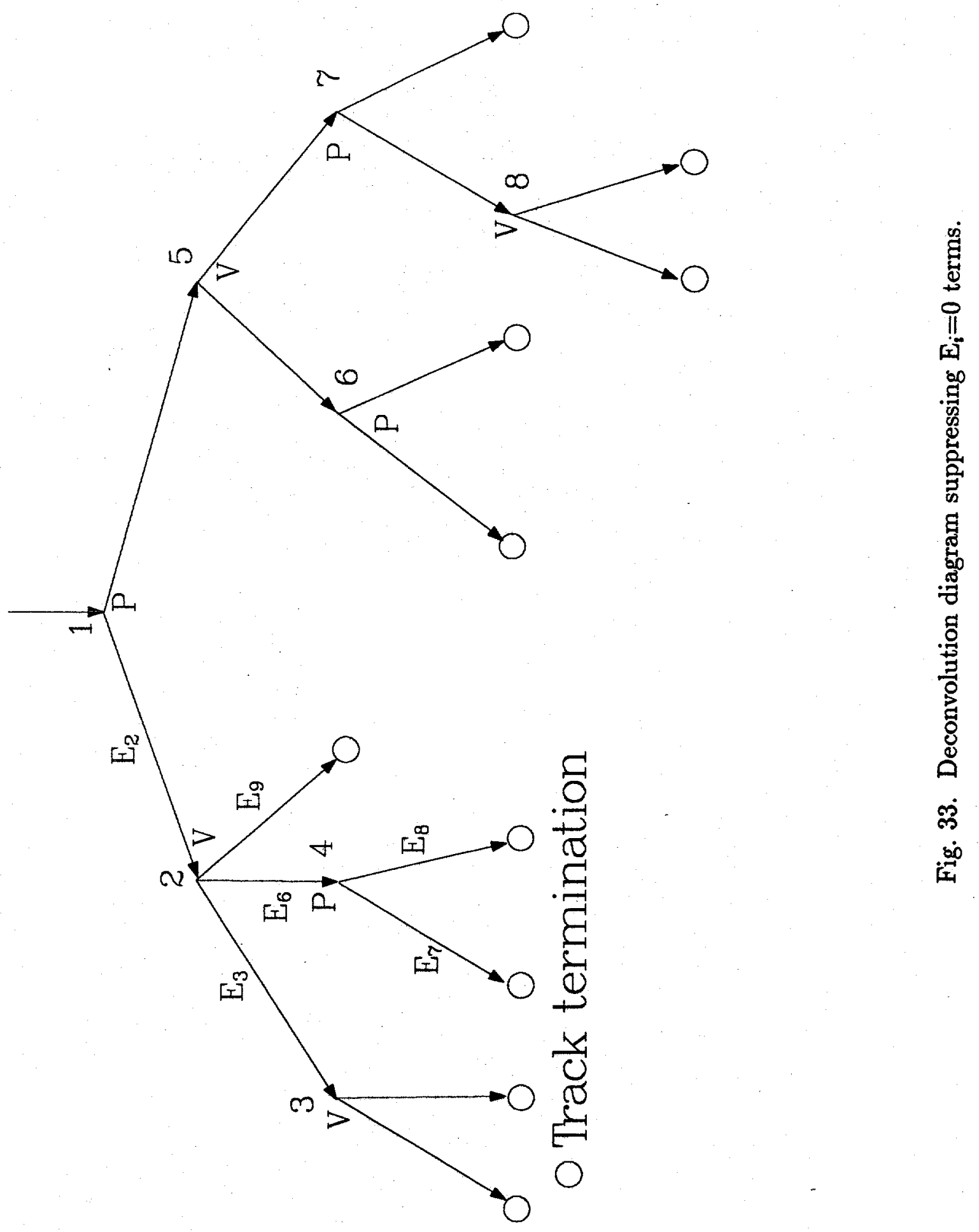



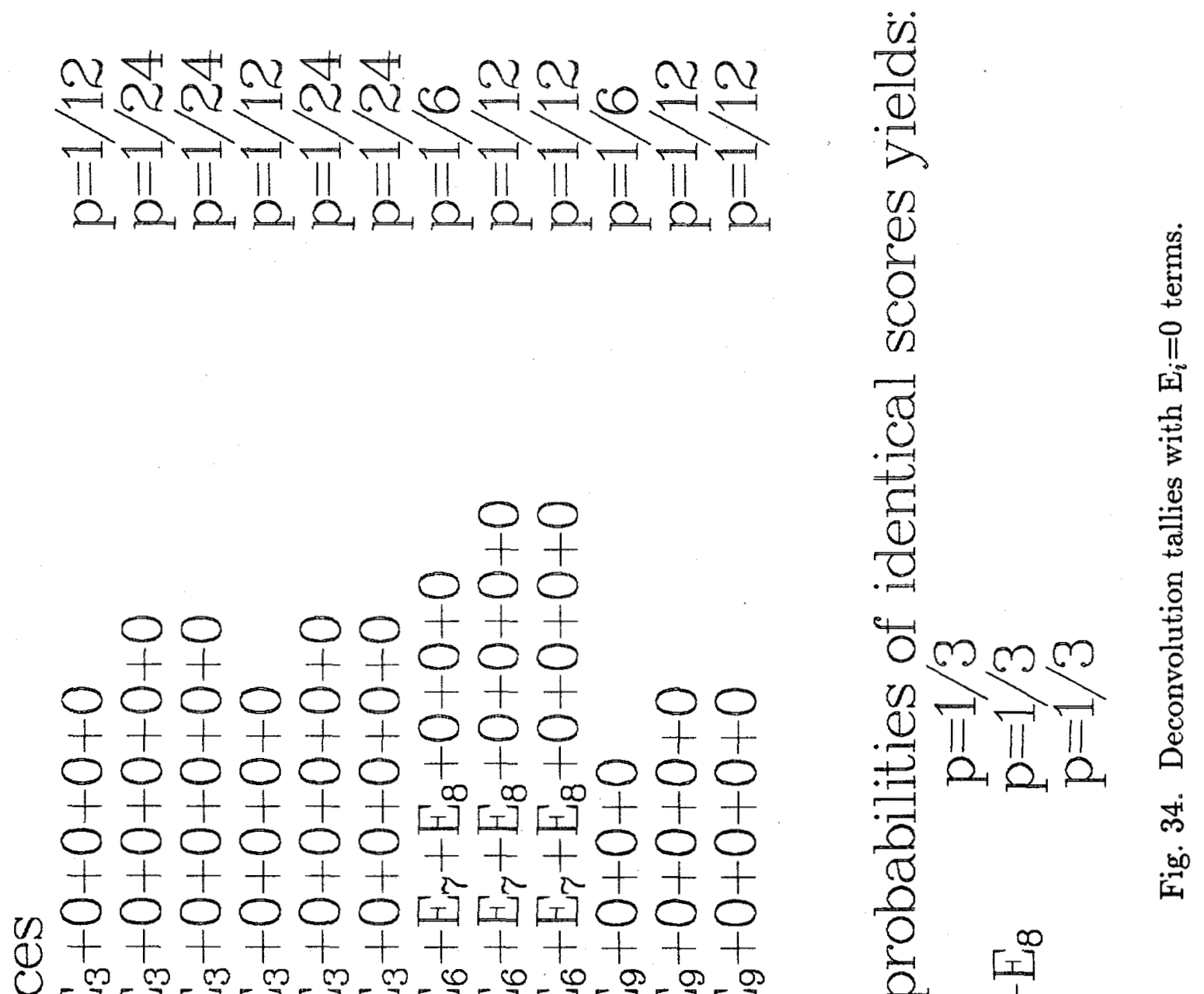

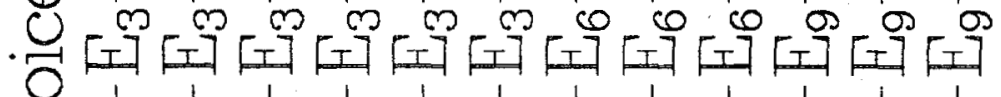

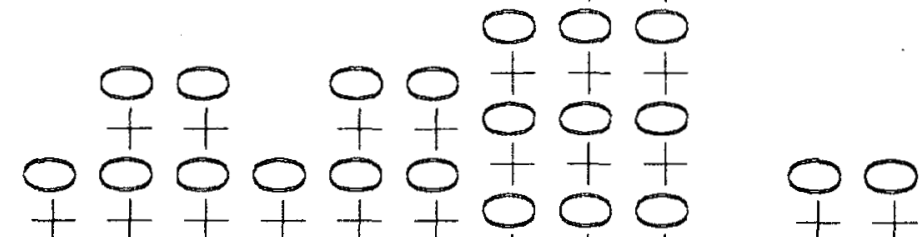

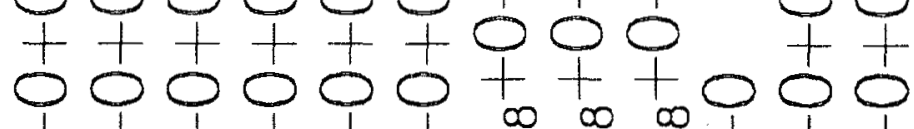

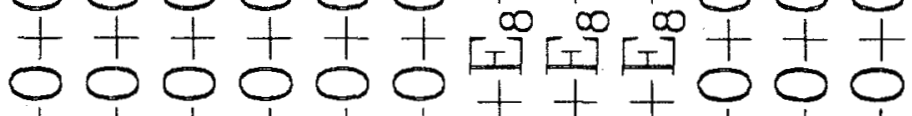

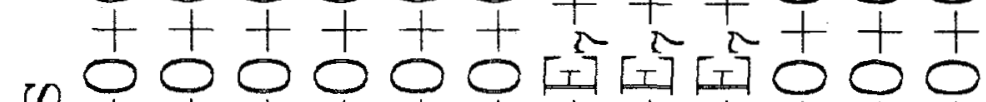

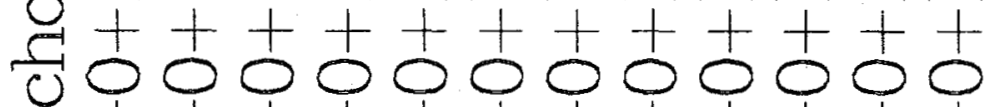
0

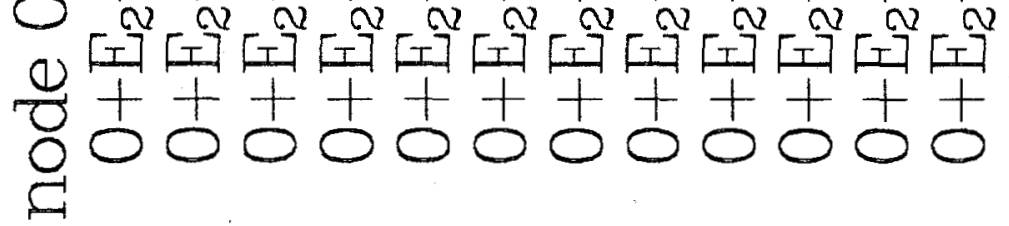

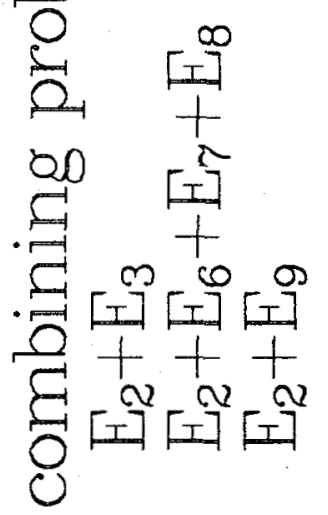




$$
E_{2}+E_{9} \quad \text { with tally }=\frac{1}{3}
$$

The difference in tallies (deconvolution minus Boltzmann) is:

$$
\begin{gathered}
E_{3}+E_{2} \quad \text { with tally difference }=0 \\
E_{7}+E_{6}+E_{2} \quad \text { with tally difference }=-\frac{1}{3} \\
E_{8}+E_{6}+E_{2} \quad \text { with tally difference }=-\frac{1}{3} \\
E_{2}+E_{6}+E_{7}+E_{8} \quad \text { with tally difference }=\frac{1}{3} \\
E_{9}+E_{2} \quad \text { with tally difference }=0
\end{gathered}
$$

Suppose that with probability $q$ the full deconvolution is done. That is, if random number $\xi>q$ then the corrected single particle approach uses the Boltzmann tallies Eqs. (3.11-3.15) (tallies from Eqs. (3.11) and (3.12) have been combined):

$$
\begin{array}{r}
E_{3}+E_{2} \quad \text { with tally }=\frac{1}{3} \\
E_{7}+E_{6}+E_{2} \quad \text { with tally }=\frac{1}{3} \\
E_{8}+E_{6}+E_{2} \quad \text { with tally }=\frac{1}{3} \\
E_{9}+E_{2} \quad \text { with tally }=\frac{1}{3}
\end{array}
$$

If $\xi<q$ then one adds $1 / q$ times a correction term (deconvolution tally minus Boltzmann tally) to the Boltzmann tallies. The tally for the energy bin containing $E_{3}+E_{2}$ is obtained from Eqs. (3.11), (3.12), and (3.19):

$$
E_{3}+E_{2} \quad \text { with tally }=\frac{1}{3}+\frac{1}{q} \cdot 0
$$


The tally for the energy bin containing $E_{7}+E_{6}+E_{2}$ is obtained from Eqs. (3.13) and (3.20):

$$
E_{7}+E_{6}+E_{2} \quad \text { with tally }=\frac{1}{3}+\frac{1}{q}\left(\frac{-1}{3}\right)
$$

The tally for the energy bin containing $E_{8}+E_{6}+E_{2}$ is obtained from Eqs. (3.14) and (3.21):

$$
E_{8}+E_{6}+E_{2} \text { with tally }=\frac{1}{3}+\frac{1}{q}\left(\frac{-1}{3}\right)
$$

The tally for the energy bin containing $E_{2}+E_{6}+E_{7}+E_{8}$ is obtained from Eq. (3.22) (the Boltzmann tally in this bin is zero):

$$
E_{2}+E_{6}+E_{7}+E_{8} \quad \text { with tally }=0+\frac{1}{q} \frac{1}{3}
$$

The tally for the energy bin containing $E_{9}+E_{2}$ is obtained from Eqs. (3.15) and (3.23)

$$
E_{9}+E_{2} \quad \text { with tally }=\frac{1}{3}+\frac{1}{q} \cdot 0
$$

\section{A. Proof of Unbiasedness with the Corrected Single Particle Approach}

To prove unbiasedness (in any particular tally bin) a few definitions are required. Define:

$\mathbf{r}=\left(r_{1}, r_{2}, r_{3}, \cdots\right)$, a random number sequence from the random number generator

$s_{b}(\mathbf{r})=$ Boltzmann score on random walk specified by $\mathbf{r}$

$s_{d}(\mathbf{r})=$ deconvolution score on random walk specified by $\mathbf{r}$

$s(\mathbf{r})=$ corrected single particle score on random walk specified by $\mathbf{r}$

$q=$ probability for doing deconvolution

Note that $s(\mathbf{r})$ is randomly assigned one of the following two values:

$$
\begin{array}{r}
s(\mathbf{r})=s_{b}(\mathbf{r}) \quad \text { with probability } 1-q \\
s(\mathbf{r})=s_{b}(\mathbf{r})+\frac{1}{q}\left(s_{d}(\mathbf{r})-s_{b}(\mathbf{r})\right) \quad \text { with probability } q
\end{array}
$$

The expected tally for the corrected single particle approach is:

$$
\int s(\mathbf{r}) d \mathbf{r}=\int\left[(1-q) s_{b}(\mathbf{r})+q\left(s_{b}(\mathbf{r})+\frac{1}{q}\left(s_{d}(\mathbf{r})-s_{b}(\mathbf{r})\right)\right] d \mathbf{r}=\int s_{d}(\mathbf{r}) d \mathbf{r}\right.
$$


Noting that the last term is the expected tally for the deconvolution approach completes the proof because the deconvolution approach preserves the analog expected tally.

\section{B. Summary of Splitting and the Corrected Single Particle Approach}

This approach shares many of the good and bad features of the deconvolution approach. Like the deconvolution approach, this approach can correctly estimate non-Boltzmann tallies (such as pulse height tallies) using the standard track splitting game typically applied to standard tallies like flux or current. This approach permits standard Monte Carlo codes to get non-Boltzmann tallies using the same particle tracking algorithms as employed for tallies such as flux. Like the deconvolution approach, this approach is also unappealing from a philosophical viewpoint because the physics is buried in the deconvolution rather than being explicitly expressed in the transport algorithm. Effective use of variance reduction techniques is usually guided by the Monte Carlo practitioner's intuitive understanding of the natural transport process and the information supplied by short scoping calculations with the Monte Carlo code. The more divorced the natural transport process becomes from the Monte Carlo algorithm, the more difficult it becomes for the practitioner to use his intuition and to interpret the information supplied by short scoping calculations.

The corrected single particle approach has advantages over the deconvolution approach because the amount of bookkeeping is significantly reduced since the bookkeeping is only done a small fraction ( $q$ above) of the time. This approach should reduce the computer time per source particle compared to the deconvolution approach. However, the optimal $q$ will likely be problem-dependent, and some $q$ 's will increase the history variance faster than the computer time per particle is decreased, resulting in a less efficient calculation when compared to the deconvolution approach (i.e., $q=1$ ).

This approach is likely to be beneficial only when the tally correction terms are small compared to the tally, but the computer coding required is essentially that of the deconvolution method. The corrected single particle approach can be used with the deconvolution approach becoming a subset $(q=1)$ of the corrected single particle approach.

\section{THE SUPERTRACK APPROACH FOR NON-BOLTZMANN TALLIES}

A supertrack represents a physical collection of particles that could be observed (at least hypothetically) in nature. An analog supertrack can be produced by a Monte Carlo code if each possible event is sampled with the natural physical probabilities so that the Monte Carlo code exactly simulates nature. Figure 1 shows an analog random walk for a supertrack. Using the subscripts on the $E$ as labels, this supertrack history is described. 
The supertrack of Fig. 1 starts at the top of the figure as a single source particle. The supertrack randomly walks for awhile and then undergoes a physical split (e.g., pair production and subsequent annihilation) at node 1. The supertrack now consists of particles 2 and 10. Particle 2 randomly walks around for awhile and undergoes a physical split at node 2. The supertrack now consists of particles 3,6 , 9 , and 10. Particle 3 randomly walks around for awhile and undergoes a physical split at node 3 . The supertrack now consists of particles $4,5,6,9$, and 10. Particle 4 randomly walks around for awhile and terminates (e.g., by absorption). The supertrack now consists of particles 5, 6, 9, and 10. Particle 5 randomly walks around for awhile and terminates. The supertrack now consists of particles 6,9 , and 10. Particle 6 randomly walks around for awhile and undergoes a physical split at node 4. The supertrack now consists of particles $7,8,9$, and 10 . Particle 7 randomly walks around for awhile and terminates. The supertrack now consists of particles 8, 9, and 10. Particle 8 randomly walks around for awhile and terminates. The supertrack now consists of particles 9 and 10. Particle 9 randomly walks around for awhile and terminates. The supertrack now consists of particle 10. Particle 10 randomly walks around for awhile and terminates. This terminates the supertrack history. If the $E_{i}$ are the energies deposited in a detector (between nodes), then for a pulse height detector, the history would score 1 in the energy channel containing $E_{1}+\cdots+E_{10}$.

The supertrack approach applies variance reduction to physical collections of tracks ("supertracks" or simply "stracks") and requires redefinition of standard Monte Carlo concepts. For example, a multiplying process (e.g., (n,2n)) does not create a new strack, but instead includes the new track in the current strack. Physical events can never make new stracks. Variance reduction methods are the only way to make more stracks. The individual particle tracks would no longer carry any weight: the variance reduction is applied to the supertracks, and thus the weights are associated with the supertracks. Termination of a strack requires termination of every track in the strack.

There are two implications of this fact. First, if a track is terminated by a physical event such as capture or escape, then the rest of the tracks in the strack continue their transport, just as happens in nature. Second, strack termination by variance reduction methods always terminates the entire strack. It is not possible to terminate just one track of a strack with variance reduction because the strack must represent a possible physical outcome.

This report considers generalizations of four nonanalog techniques used in MCNP:

1. Russian roulette

2. implicit capture

3. splitting

4. dxtran 


\section{A. Russian Roulette with the Supertrack Approach}

Consider a Russian roulette game with a survival probability $p$. Suppose that the strack (weight $w$ ) consists of five tracks. The possible outcomes of this roulette game are:

1. a strack of weight $w / p$ consisting of five tracks, or

2. a strack of weight 0 consisting of five tracks (i.e., termination by roulette).

Russian roulette can thus be played when the strack is no longer interesting enough to follow at low weight. Russian roulette must be played on the entire strack because it is the strack that is the physical quantity and it is the strack that carries the weight. Individual tracks of a strack do not have individual weights. Only the collection has a weight. The entire strack either survives or dies.

Consider what would happen if this were not so. If roulette were played on only one track (of the strack above), it could result in a strack that consists of four tracks. This result is impossible because no physical termination has occurred. Thus, there must still be five tracks.

\section{B. Implicit vs. Analog Capture with the Supertrack Approach}

One can generalize implicit capture from tracks to stracks, but it is not clear whether this generalization is useful. Part of the problem is that capture does not happen to a strack in the same sense that capture happens to a track. In trackbased Monte Carlo, an analog sampling for survival versus absorption either results in one track or in zero tracks. Now consider a strack consisting of $k$ tracks. Only one track of the strack collides at a time. An analog sampling for survival versus absorption either results in one strack with $k$ tracks or one strack with $k-1$ tracks. Thus, (except when $k=1$ ) the analog capture sampling does not result in the loss of the strack. Thus, an implicit capture technique may not have the same usefulness as in track based Monte Carlo.

Suppose that an implicit capture technique is desired. There are a number of ways that the technique might be specified. This paper considers three possible specifications (this is not an exhaustive list)

1. Play implicit capture only if $k=1$

2. Wait until all tracks of the strack have entered collision (or have terminated) and use implicit capture to ensure that at least one track survives.

3. When a track of a strack collides, the strack is divided into two stracks: one strack in which the track survives the collision and one strack in which the colliding track is absorbed.

The first case can be treated exactly as currently done because the implicit capture is only being applied when the track and the strack are the same.

In the second case suppose that the $k$ tracks in the strack have absorption probabilities $p_{i}$. The probability that all tracks of the strack would be absorbed is $p_{1} p_{2} \cdots p_{k}$. Thus, if the strack has weight $w$ immediately before the collision then 
the implicitly captured strack weight is $w p_{1} p_{2} \cdots p_{k}$ and the surviving strack weight is $w\left(1-p_{1} p_{2} \cdots p_{k}\right)$. The surviving strack is then sampled from the conditional density that excludes all tracks being absorbed. (This density can easily be sampled by rejecting the surviving strack sampling if all tracks are absorbed and then resampling.)

In the third case suppose that the $i^{\text {th }}$ track has just collided. The strack is the split into two stracks. The "implicitly absorbed" strack will then consist of tracks $1,2 \cdots, i-1, i+1, \ldots, k$ with weight $w p_{i}$ and the "surviving" strack will have the full $k$ tracks but with its weight reduced to $w\left(1-p_{i}\right)$.

These are but three of the many possibilities. These methods are all unbiased, but their usefulness is unclear.

A variance reduction termination is equivalent to setting the weight equal to zero because a sample of weight 0 cannot contribute to any tally and thus the sample's tallies are known without continuing its random walk. In standard track-based Monte Carlo, the weight is associated with a track, and thus a track is terminated when it's weight is zero. In strack-based Monte Carlo, the weight is associated with a strack, and thus an entire strack is terminated when its weight is zero.

\section{Splitting with the Supertrack Approach}

A supertrack represents a physical collection of particles that can collectively contribute to a tally. As such, any supertrack consists of a collection of $n$ particles that have phase-space coordinates

$$
\mathbf{P}=\left(\mathbf{r}_{1}, \mathbf{v}_{1}, t_{1}, \cdots, \mathbf{r}_{n}, \mathbf{v}_{n}, t_{n}\right)
$$

where $\left(\mathbf{r}_{k}, \mathbf{v}_{k}, t_{k}\right)$ is the $k^{\text {th }}$ particle's position, velocity, and time. An $m: 1$ split means replacing a supertrack of weight $w_{0}$ at $\mathbf{P}$ by $m$ supertracks with weights $w_{0} / m$ at $\mathbf{P}$. For example, a $2: 1$ split results in:

$$
\begin{aligned}
& \mathbf{P}_{1}=\left(\mathbf{r}_{1}, \mathbf{v}_{1}, t_{1}, \cdots, \mathbf{r}_{n}, \mathbf{v}_{n}, t_{n}\right) \quad \text { with } w_{1}=\frac{w_{0}}{2} \\
& \mathbf{P}_{2}=\left(\mathbf{r}_{1}, \mathbf{v}_{1}, t_{1}, \cdots, \mathbf{r}_{n}, \mathbf{v}_{n}, t_{n}\right) \quad \text { with } w_{2}=\frac{w_{0}}{2}
\end{aligned}
$$

Figure 35 shows a $2: 1$ split pictorially for a strack consisting of three tracks.

Suppose that at the time of the split the total energy deposited in the detector so far was $E_{0}$, and suppose that the simulation was analog except for this single split. Suppose that split strack $i$ subsequently deposits $E_{i}$ in the detector. Thus, the total energy deposited by strack $i$ is $E_{0}+E_{i}$ with weight $w_{0} / 2$. The pulse height tally associated with the history is:

$$
\frac{w_{0}}{2} \quad \text { in the energy bin containing } \quad E_{0}+E_{1}
$$




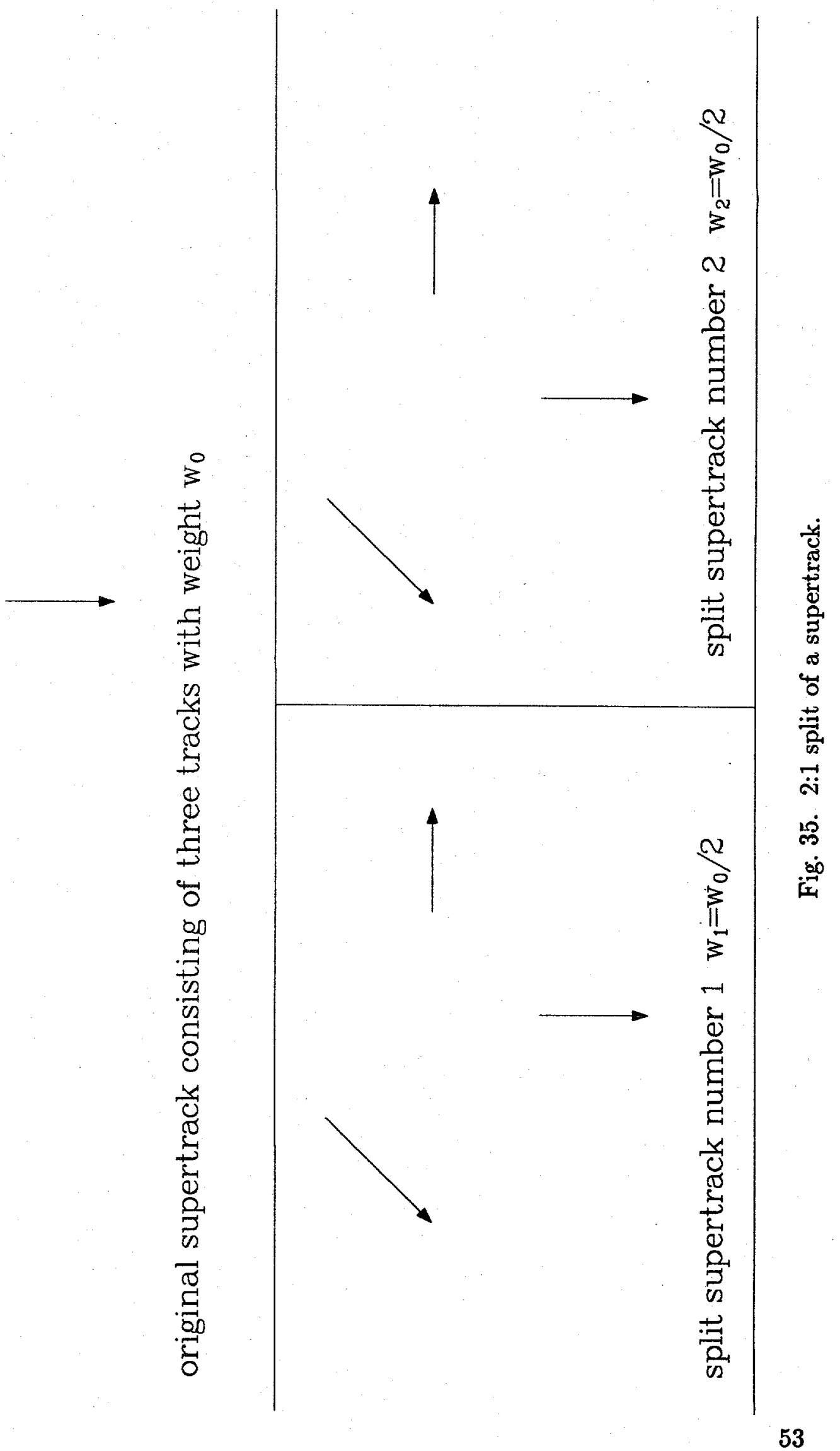




$$
\frac{w_{0}}{2} \quad \text { in the energy bin containing } \quad E_{0}+E_{2}
$$

\section{Proof that the Expected Tally is Preserved with Splitting}

Proving that the expected tally is preserved with splitting requires showing that integrations (over all scores) of the score pdf times the score (i.e., the means) in both the split and no-split are the same. To do this, the proof writes the pdf for the split case in terms of the pdf for the no-split case. Note that the pdf for the no-split case is not necessarily an analog pdf. There may be many nonanalog samplings following the split/no-split decision. The no-split pdf is simply the density of scores produced (in the absence of a split at the current point) by whatever sampling procedure is subsequently applied.

To prove that the splitting technique provides unbiased estimates note that (if no weight-dependent games are played):

$$
p\left(s w_{1}, w_{1}\right) d\left(s w_{1}\right)=p\left(s w_{2}, w_{2}\right) d\left(s w_{2}\right)=p(s, 1) d s
$$

where

$p(s, w) d s=$ probability that an strack of weight $w$ contributes a score in $d s$ about $s(4.5)$

That is, a strack of weight $w_{1}$ must produce a score in $d\left(s w_{1}\right)$ about $s w_{1}$ with the same probability that a strack of weight 1 produces a score in $d s$ about $s$. If no weight-dependent games are played, then this condition is satisfied. This fact can be understood by noting that if no weight-dependent games are played, then the random walks are independent of weight. Thus, two otherwise identical stracks with different weights will execute exactly the same random walk (i.e., they will "track") for any given random number sequence, and the scores produced will always be the same except for a constant weight factor.

Suppose that one is considering whether a single 2:1 split preserves the mean or not. Let $p(s, w)$ be the score pdf (for the score produced after the split/no-split decision) for the strack in the absence of the split, but with any arbitrary collection of weight-independent variance reduction techniques employed after the decision. The score pdf for each of the split stracks is $p(s, v)$ where $v=w / 2$. Because the split stracks are sampled independently, $p\left(s_{1}, v\right) p\left(s_{2}, v\right) d s_{1} d s_{2}$ is the probability that $s_{1}$ is in $d s_{1}$ and $s_{2}$ is in $d s_{2}$. Thus, the probability that the collective score (for two stracks of weight $v$ ) is in $d s$ about $s$ is

$$
p_{2}(s, v) d s=\iint \delta\left(s-\left(s_{1}+s_{2}\right)\right) d s p\left(s_{1}, v\right) p\left(s_{2}, v\right) d s_{1} d s_{2}
$$

where the $\delta$ function constrains the sum of the scores from both split stracks to be $s$ in $d s$. The expected score in the split case is 


$$
\begin{aligned}
& E_{s p l i t}=\int p_{2}(s, v) s d s=\iint p\left(s_{1}, v\right) p\left(s_{2}, v\right)\left(s_{1}+s_{2}\right) d s_{1} d s_{2} \\
& E_{s p l i t}=\int p\left(s_{1}, v\right) s_{1} d s_{1} \int p\left(s_{2}, v\right) d s_{2}+\int p\left(s_{2}, v\right) s_{2} d s_{2} \int p\left(s_{1}, v\right) d s_{1}
\end{aligned}
$$

Now using the fact that $p(s, v)$ is a pdf, Eq. (4.8) yields

$$
E_{s p l i t}=2 \int p(s, v) s d s
$$

Changing variables $s=t / 2$, recalling that $v=w / 2$, and using the equation in the preceding paragraph yields

$$
E_{s p l i t}=2 \int p\left(\frac{t}{2}, \frac{w}{2}\right) \frac{t}{2} d \frac{t}{2}=\int p(t, w) t d t=E
$$

where $E$ is the expected score in the no split case.

In the general case of an $n: 1$ split let:

$$
\begin{aligned}
& p_{n}(s, \eta) d s=\text { probability that } n \text { stracks each of weight } \eta \text { collectively score } \\
& \text { in } d s \text { about } s
\end{aligned}
$$

$$
\begin{aligned}
& E=\int p(s, w) s d s=\text { the expected tally in the no split case } \\
& E_{n}=\int p_{n}(s, \eta) s d s=\text { the expected tally in the split case }
\end{aligned}
$$

Changing variables $s=w y$ and using Eq. (4.4)

$$
E=\int p(w y, w) w y d(w y)=w \int p(y, 1) y d y
$$

Because the split stracks are sampled independently,

$$
\prod_{j=1}^{n} p\left(s_{j}, \eta\right) d s_{j}=\text { probability that } s_{j} \text { is in } d s_{j} \text { about } s_{j} \quad \text { for } j=1,2, \ldots, n
$$

Thus, the probability that the score from all split stracks together is in $d s$ about $s$ is (the $\delta$ function constrains the sum to be $s$ in $d s$ ): 


$$
p_{n}(s, \eta) d s=\int \cdots \int \delta\left(s-\sum_{k=1}^{n} s_{k}\right) d s \prod_{j=1}^{n} p\left(s_{j}, \eta\right) d s_{1} \cdots d s_{n}
$$

Substituting Eq. (4.16) into Eq. (4.13) above

$$
E_{n}=\int p_{n}(s, \eta) s d s=\iint \cdots \int \delta\left(s-\sum_{k=1}^{n} s_{k}\right) \prod_{j=1}^{n} p\left(s_{j}, \eta\right) d s_{1} \cdots d s_{n} s d s
$$

Performing the indicated integration over $s$

$$
E_{n}=\int \cdots \int \sum_{k=1}^{n} s_{k} \prod_{j=1}^{n} p\left(s_{j}, \eta\right) d s_{1} \cdots d s_{n}
$$

Interchanging sum and integrals

$$
E_{n}=\sum_{k=1}^{n} \int \cdots \int s_{k} \prod_{j=1}^{n} p\left(s_{j}, \eta\right) d s_{1} \cdots d s_{n}
$$

Because the $p$ 's are probability densities:

$$
\begin{gathered}
\int p\left(s_{j}, \eta\right) d s_{j}=1 \\
E_{n}=\sum_{k=1}^{n} \int p\left(s_{k}, \eta\right) s_{k} d s_{k}=n \int p(y, \eta) y d y
\end{gathered}
$$

Changing variables $y=\eta s$

$$
E_{n}=n \int p(\eta s, \eta) \eta s d \eta s
$$

Now using Eq. (4.4), recalling that the split weight is $\eta=w / n$, and noting Eq. (4.14) yields

$$
E_{n}=n \int p(s, 1) \eta s d s=w \int p(s, 1) s d s=E
$$




\section{E. A Dxtran Method with the Supertrack Approach}

The dxtran method is one of the most complicated variance reduction methods in major Monte Carlo transport codes. In addition, the dxtran method is one of the most useful methods for getting particles to an isolated pulse height detector region. For these reasons, a mathematical analysis and practical demonstration of a dxtran method with the supertrack approach is extremely important. Not only will the demonstration indicate that complicated variance reduction techniques can be generalized for the supertrack approach, it also provides a practical means for getting particles to the detector region.

The standard dxtran method ${ }^{1}$ partitions the space of possible next events, $\Omega$, into two mutually exclusive sets $A$ and $B$ such that $A \cup B=\Omega$. Set $B$ is associated with sampling the dxtran sphere and set $A$ is associated with sampling stracks that do not reach the dxtran sphere. Let $\mathbf{x}$ be a possible next event and let $p(\mathbf{x})$ be its analog pdf. That is,

$$
\int_{\mathbf{x} \in \Omega} p(\mathbf{x}) d \mathbf{x}=1
$$

Let $q(\mathbf{x})$ be an arbitrary nonzero pdf on set $B$ such that

$$
\begin{aligned}
& q(\mathbf{x})=0 \quad \quad \mathbf{x} \in A \\
& \int_{\mathbf{x} \in B} q(\mathbf{x}) d \mathbf{x}=1
\end{aligned}
$$

Typically, $B$ will be sampled very rarely, and it is desired to sample $B$ more frequently. To solve this problem, two samples are taken instead of one. First, the dxtran method samples an event $x \in B$ from $q(\mathbf{x})$ (the dxtran sample) and then weights this sample by

$$
w_{d}(\mathbf{x})=\frac{\text { true probability }}{\text { sampled probability }}=\frac{p(\mathbf{x}) d \mathbf{x}}{q(\mathbf{x}) d \mathbf{x}} .
$$

Second, the dxtran method samples an event $x \in \Omega$ from $p(x)$ (the nondxtran sample) and accepts the sample if $\mathbf{x} \notin B$ (i.e., $\mathbf{x} \in A$ ) and kills the sample if $\mathbf{x} \in B$.

The dxtran method preserves the expected weight executing any next event. To see this, note that the expected weight in $d \mathbf{x}$ about $\mathbf{x}$ due to the dxtran sample plus the expected weight in $d \mathbf{x}$ about $\mathbf{x}$ due to the nondxtran sample is

$$
\begin{aligned}
w_{0} w_{d}(\mathbf{x}) q(\mathbf{x}) d \mathbf{x}+0 & =w_{0} p(\mathbf{x}) d \mathbf{x} & & \text { for } \mathbf{x} \in B \\
0+w_{0} p(\mathbf{x}) d \mathbf{x} & =w_{0} p(\mathbf{x}) d \mathbf{x} & & \text { for } \mathbf{x} \in A
\end{aligned}
$$

But $w_{0} p(\mathbf{x}) d \mathbf{x}$ is the expected analog weight in $d \mathbf{x}$ about $\mathbf{x}$, so the method is unbiased. 
Although it is possible to get more than one photon coming out of a collision (with pair production and double fluorescence), the simplest way to explain supertrack dxtran is to consider a single photon emerging from a collision. After the single photon emerging case has been explained, then dxtran with pair production and with double fluorescence will be explained.

Suppose that one of $k$ photons of a strack has a collision in which dxtran is employed. Label this photon with a 1 . Note that applying dxtran at \#1's collision changes \#1's phase-space coordinates, but the other $k-1$ photons do not change their phase-space coordinates. Thus, the possible outcomes differ only in the phasespace coordinates of the first photon. The partition of the space of possible next events (for the first photon) is shown in Fig. 36. There are three possible types of physical next events:

1. The photon can escape the system, see $P_{1}$ in Fig. 36 .

2. The photon can collide anywhere inside the escape surface (except in the forbidden zone behind the dxtran sphere), see $P_{2 a}$ and $P_{2 b}$ in Fig. 36 .

3. The photon can reach the front dxtran surface without collision, see $P_{3}$ in Fig. 36.

Set $A$ contains all possible escape points that can occur as a next event (type 1) and all possible collision points that can occur as a next event (type 2). Set $B$ contains all possible crossings of the front dxtran surface that can occur as a next event (type 3). Note that events cannot occur (to photon \#1) in the "forbidden zone" unless the photon first crosses the dxtran sphere. Thus, it is impossible for any next events to occur in the forbidden zone.

In the case of a single emerging photon, the dxtran method thus proceeds as follows:

1. A dxtran photon $(x \in B)$ is sampled from the set of photons that have next events that cross the front surface of the dxtran sphere without collision. (This sampling is from an arbitrary and easily sampled density; a two-step histogram is used in MCNP) After the event has been sampled, the true density for that event is calculated and the dxtran strack is weighted as in Eq. (4.26).

2. A nondxtran photon $(x \in \Omega)$ is sampled in the normal manner and transported to its next event. That is, the nondxtran photon is sampled from exactly the same density, $p(\mathbf{x})$, that the original photon would have been sampled from if dxtran were not used. The nondxtran strack is killed if the photon's next event is a crossing of the dxtran sphere because this result is in set $B$ and has been accounted for in step 1 .

A simple example with the full strack shown may clarify the process. Figures 37 and 38 show a strack of weight $w_{0}$ consisting of three tracks. The leftmost track has had a collision and is currently being followed to its next collision point. The top frames of the two figures show the possible options for an analog sampling of the strack (without dxtran). That is, the leftmost track either does or does not reach 


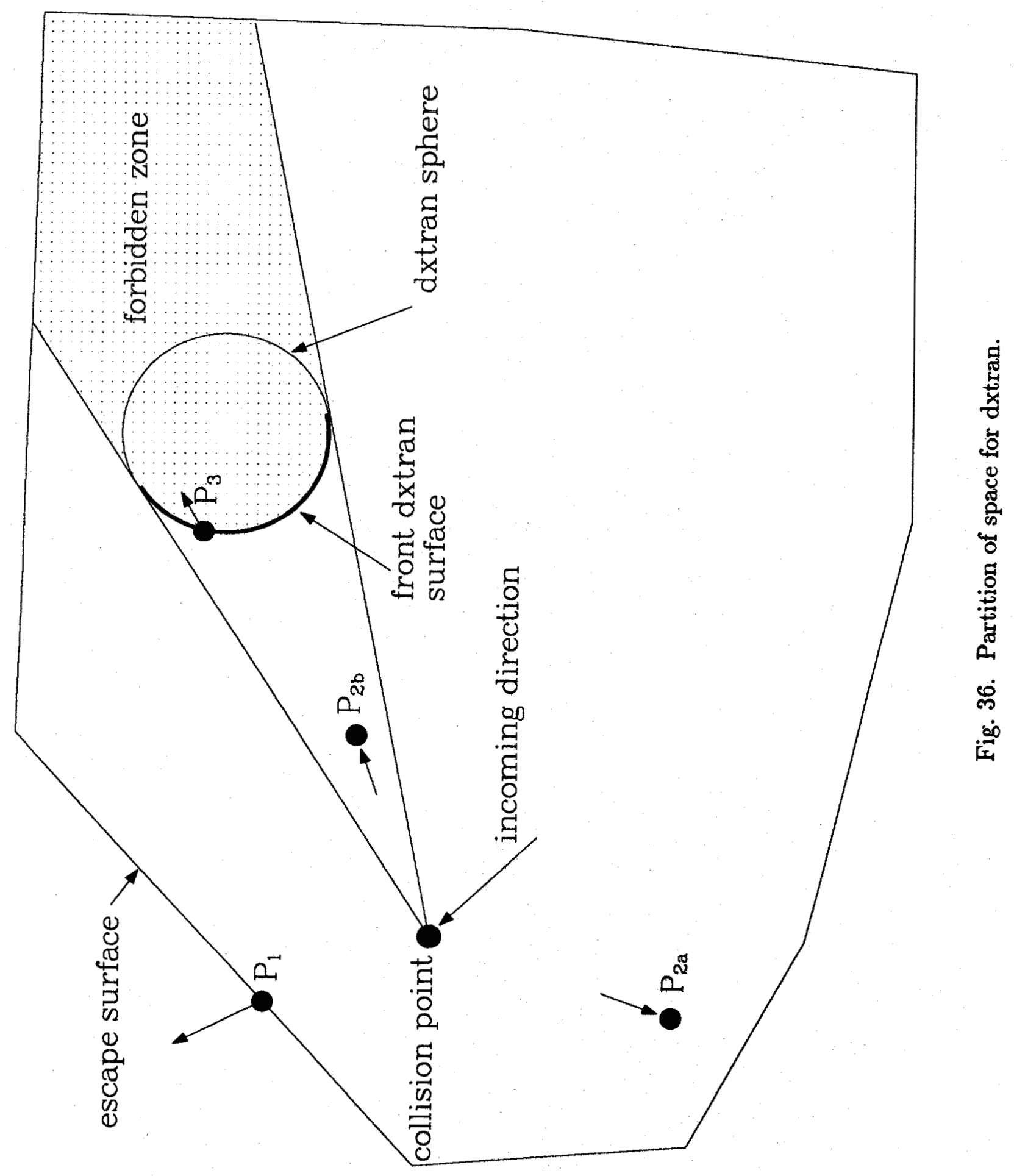




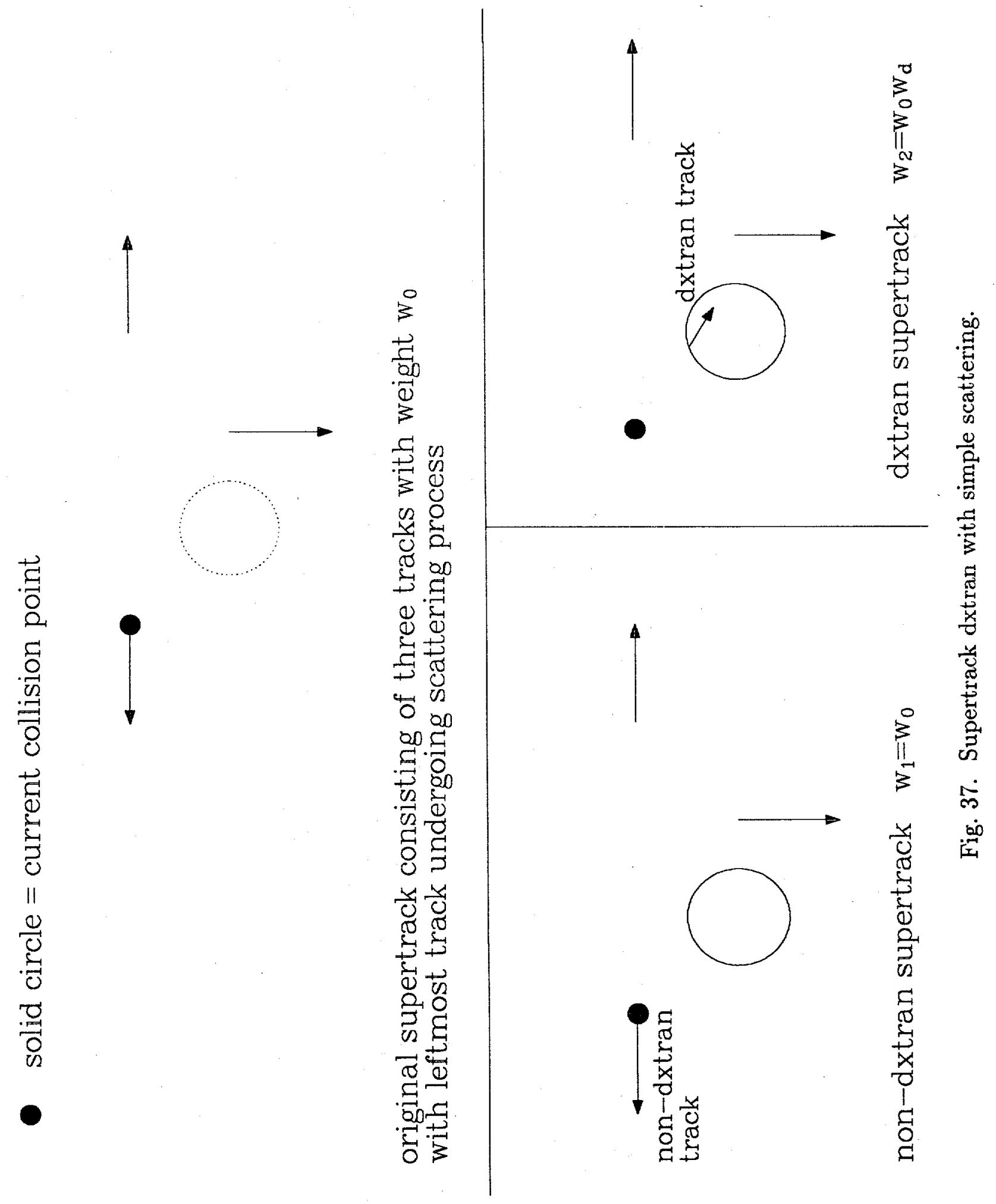




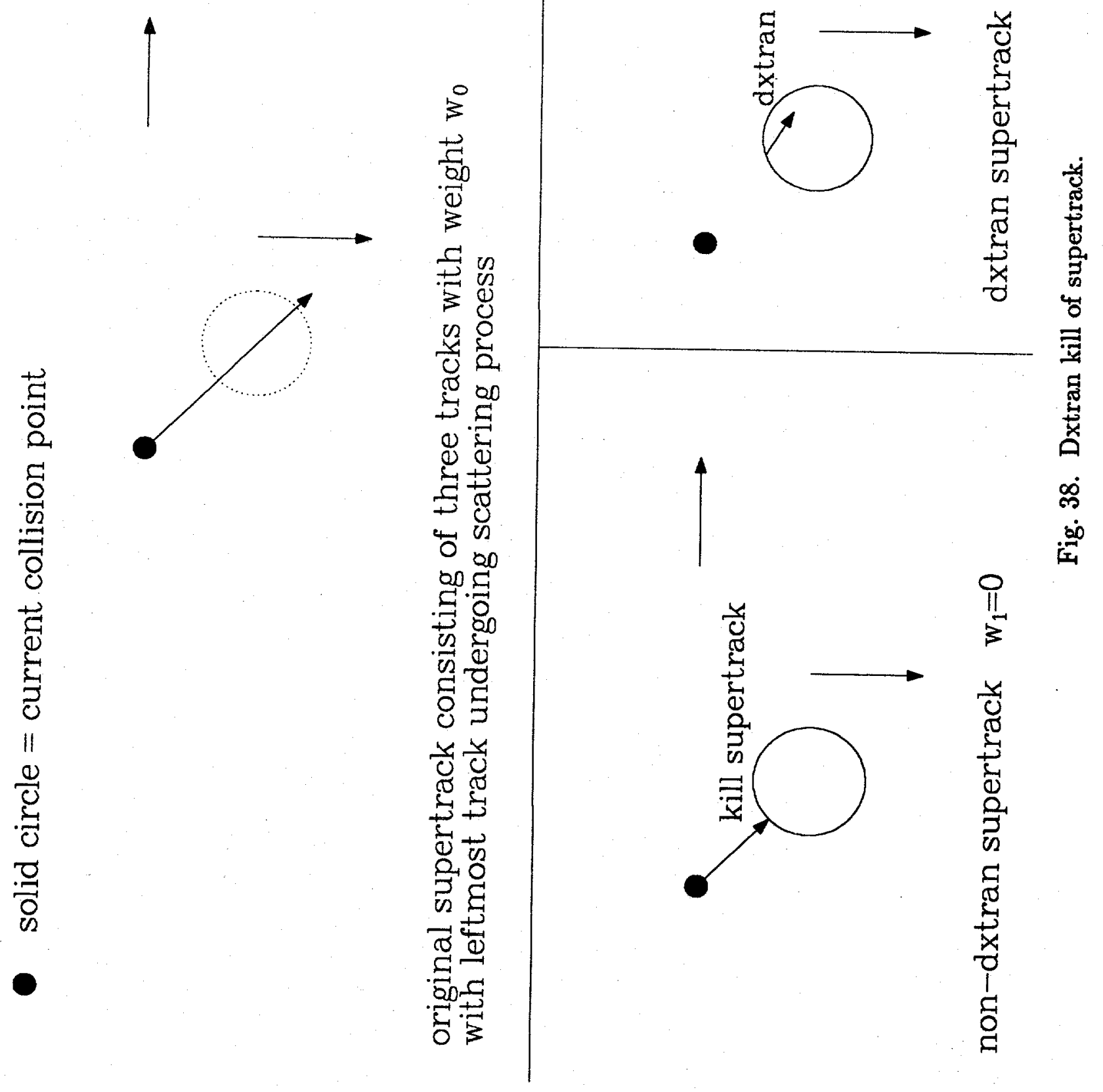


the dxtran sphere before its next event. In the top frames the current collision sampling is analog, and the dxtran sphere is shown for reference only; whereas in the bottom frames the dxtran sphere is used to produce dxtran tracks and kill some nondxtran tracks. The bottom frames of both figures show the supertrack dxtran sampling. The original strack is split into a nondxtran strack and a dxtran strack. The dxtran strack represents the number of physical collections of photons that had the leftmost photon reach the dxtran sphere without collision. The nondxtran strack represents the number of physical collections of photons that had the leftmost photon not reach the dxtran sphere. Note (Fig. 38) that if the leftmost photon of the nondxtran strack crosses the dxtran sphere, then the entire strack is killed. The dxtran strack includes the rightmost two photons.

Another way to understand why the entire nondxtran strack is killed when its leftmost photon crosses the dxtran sphere is to consider the physical possibilities as depicted in the top (analog) frames of Figs. 37 and 38. Note that in both figures the physical system consists of three photons. There is no physical possibility of just killing the leftmost photon and leaving only two photons. Killing the entire strack when the nondxtran photon crosses the dxtran sphere is compensated by creating an entire strack in the dxtran process.

\section{F. Dxtran with Pair Production Photons}

The dxtran method with two pair production photons is similar to the single photon emerging case except that there are two ways that one photon can arrive at the dxtran sphere. Label the photons from a pair production 1 and 2. There are three mutually exclusive possible physical occurrences for this pair production event:

1. Photon 1 arrives at the dxtran sphere and photon 2 does not.

2. Photon 2 arrives at the dxtran sphere and photon 1 does not.

3. Neither photon arrives at the dxtran sphere.

Note that the pair production photons exit the collision site in opposite directions so that it is impossible for both photons to arrive at the dxtran sphere.

The top frames of Figs. 39 and 40 show a pair production sampling without dxtran and the bottom frames show the pair production with dxtran. In Fig. 39 the nondxtran pair misses the dxtran sphere and thus is not killed. (Note that because of items 1 and 2 above, there are two physical possibilities that put weight on the dxtran sphere. The two photons from the pair production are identical, so that the probability density that one of the pair of photons makes it to a point on the dxtran sphere is simply double the probability density from a single photon.) In Fig. 40 one of the nondxtran pair hits the dxtran sphere and thus the strack is killed.

In terms of the dxtran discussion of the previous section, let $\mathbf{x}=(\mathbf{y}, \mathbf{z})$ be the set of possible next events for the pair of photons. That is, $p(\mathbf{x}) d \mathbf{x}=$ probability that photon pair has its next (pair) event in dx about $\mathbf{x}$. 


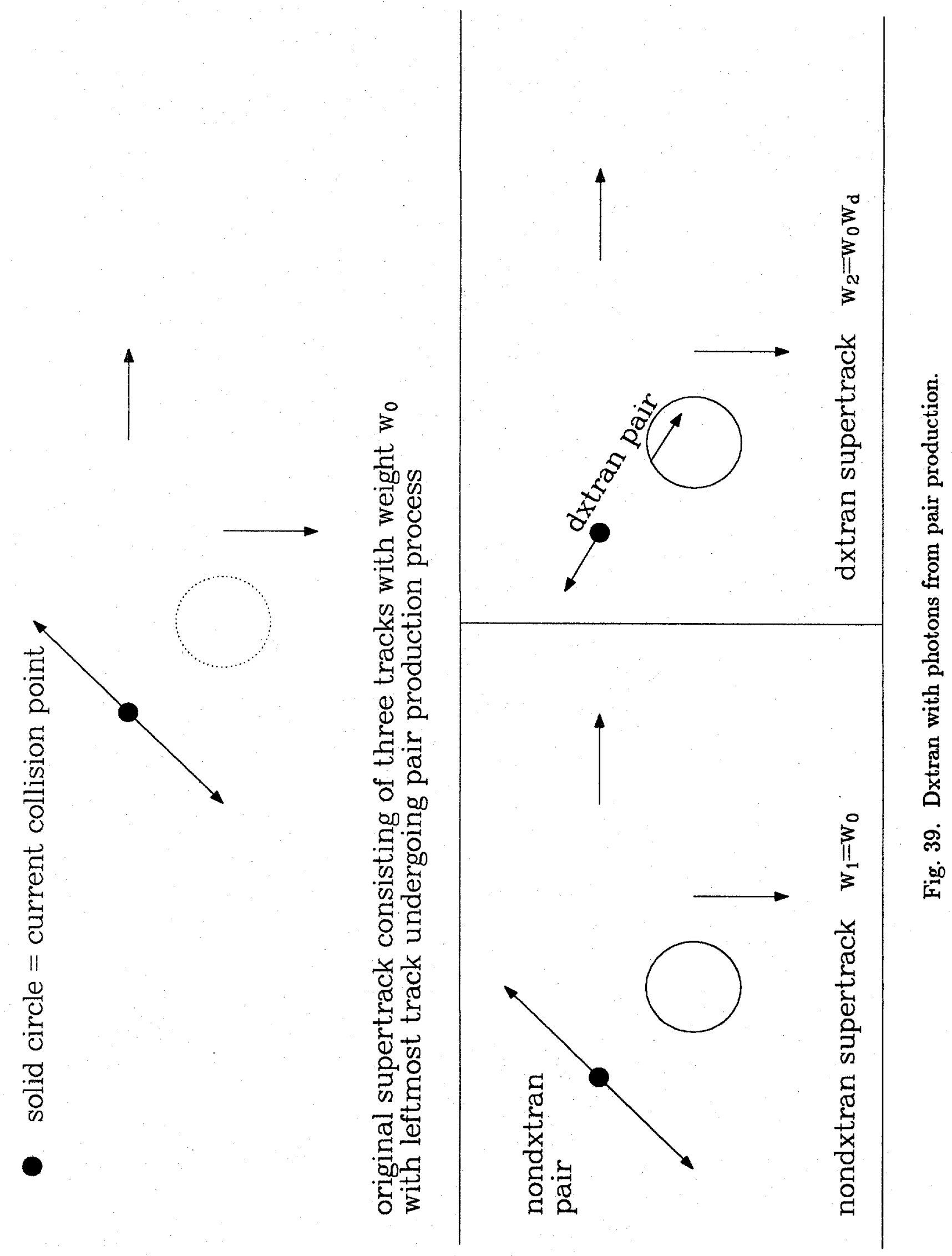




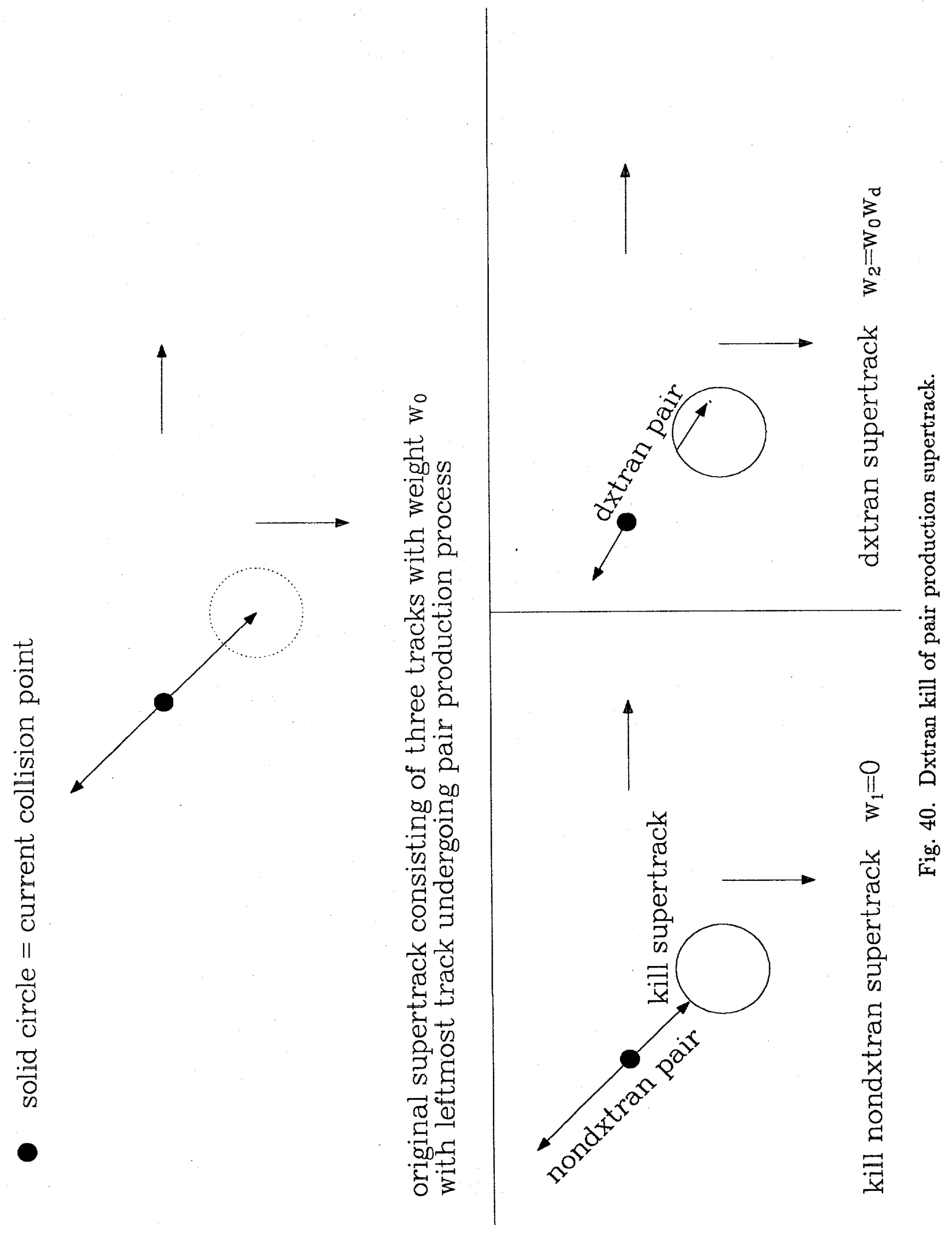


Note that this density is independent of order; photons 1,2 could be at $(\mathbf{y}, \mathbf{z})$ or photons 2,1 could be at $(\mathbf{y}, \mathbf{z})$ and the pair would be at $\mathbf{x}$.

Suppose that one of $k$ photons of a strack has a collision in which dxtran is employed. Label this photon with a 1 . Note that applying dxtran at \#1's collision changes \#1's phase-space coordinates, but the other $k-1$ photons do not change their phase-space coordinates. Thus, the possible outcomes for the strack differ only in the outcomes for photon \#1.

Set $A$ contains all possible photon pair samplings in which neither photon has a crossing of the dxtran sphere as its next event. Set $B$ contains all possible photon pair samplings in which one photon has a crossing of the dxtran sphere as its next event.

In the pair production case, the dxtran method thus proceeds as follows:

1. A dxtran photon pair $(x \in B)$ is sampled from the set of pairs that has one photon crossing the front surface of the dxtran sphere without collision as its next event. This sampling is from the arbitrary density $q(\mathbf{x})$. After the event has been sampled, the true density, $p(x)$, for that event is calculated, and the dxtran strack is weighted as in Eq. (4.26).

2. A nondxtran photon pair $(x \in A)$ is sampled in the normal manner and transported to their next events. That is, the nondxtran photon pair is sampled from exactly the same density, $p(\mathbf{x})$, that the original photon pair would have been sampled from if dxtran were not used. The nondxtran strack is killed if one of the pair's next events is a crossing of the dxtran sphere because this result is in set $B$ and has been accounted for in step 1 .

With the above definitions of $\mathbf{x}$, set $A$, and set $B$, the method is unbiased by the proof in the previous section.

\section{G. Dxtran with Double Fluorescence Photons}

The dxtran method with double fluorescence photons is different from the pair production case because the photons are not identical nor are they emitted in opposite directions. There are four mutually exclusive events:

1. Photon 1 arrives at the dxtran sphere and photon 2 does not.

2. Photon 2 arrives at the dxtran sphere and photon 1 does not.

3. Both photons arrive at the dxtran surface.

4. Neither photon arrives at the dxtran sphere.

These four possibilities are shown in Fig. 41. The set $A$ consists of item 4 above and the sets $B_{1}, B_{2}$, and $B_{3}$ consist of items 1-3, respectively. In the simple one exiting photon case there was only one set $(B)$ that was difficult to sample and now there are three sets $B_{1}, B_{2}$, and $B_{3}$ that are difficult to sample. The strack associated with the double fluorescence event is thus divided into four possible stracks, with 


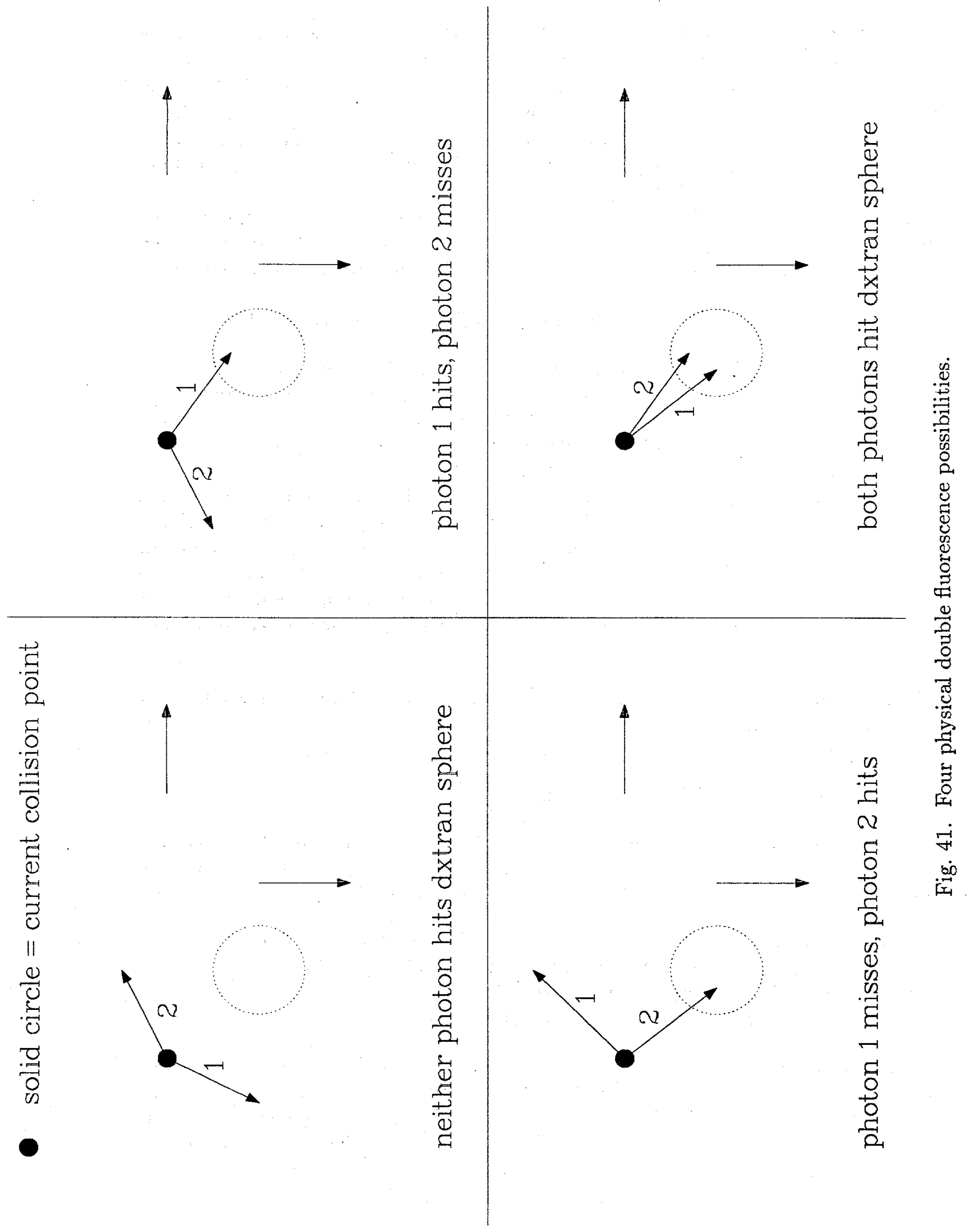


items 1-3 using dxtran to bring photons from the double fluorescence event to the dxtran sphere. Let $\mathbf{x}=(\mathbf{y}, \mathbf{z})$ represent the next event coordinates for the pair of photons from the double fluorescence, where $y$ and $z$ are the next event coordinates for photons 1 and 2 respectively. The double fluorescence photons are sampled independently so that if $f(\mathbf{y})$ and $g(\mathbf{z})$ are the original densities for $\mathbf{y}$ and $\mathbf{z}$ then

$$
p(\mathbf{x})=f(\mathbf{y}) g(\mathbf{z})
$$

The dxtran method samples the four strack possibilities as follows:

1. Photon 1 is sampled from an arbitrary density $q(\mathbf{y})$ that is nonzero only on the front surface of the dxtran sphere and photon 2 is sampled from its original density $g(\mathbf{z})$. The strack is weighted by

$$
w_{d}(\mathbf{x})=(f(\mathbf{y}) g(\mathbf{z})) /(q(\mathbf{y}) g(\mathbf{z}))
$$

as in Eq. (4.26). The strack is killed if photon 2 enters the dxtran sphere because this event is accounted for in item 3 .

2. Photon 2 is sampled from an arbitrary density $h(\mathbf{z})$ that is nonzero only on the front surface of the dxtran sphere and photon 1 is sampled from its original density $f(y)$. The strack is weighted by

$$
w_{d}(\mathbf{x})=(f(\mathbf{y}) g(\mathbf{z})) /(f(\mathbf{y}) h(\mathbf{z}))
$$

as in Eq. (4.26). The strack is killed if photon 1 enters the dxtran sphere because this event is accounted for in item 3 .

3. Photon 1 is sampled from an arbitrary density $q(y)$ that is nonzero only on the front surface of the dxtran sphere and photon 2 is sampled from an arbitrary density $h(\mathbf{z})$ that is nonzero only on the front surface of the dxtran sphere. The strack is weighted by

$$
w_{d}(\mathbf{x})=(f(\mathbf{y}) g(\mathbf{z})) /(q(\mathbf{y}) h(\mathbf{z}))
$$

4. Both photons are sampled from their original densities $f(y)$ and $g(z)$ and the strack is killed if either photon tries to enter the dxtran sphere as its next event. (These events are accounted for in items 1-3.)

As in Eq. (4.27) only one of the double fluorescence pairs occurs in each of the four sets. Thus, there will be three 0 contributions and one nonzero contribution to the expected weight at each point $x$. The expected weight arriving at any point $\mathbf{x}$ is the probability of coming to $x$ times the weight arriving, thus using Eqs. (4.30-4.32) for $w_{d}(\mathbf{x})$ yields:

$$
w_{0} w_{d}(\mathbf{x}) q(\mathbf{y}) g(\mathbf{z})+0+0+0=w_{0} f(\mathbf{y}) g(\mathbf{z})=w_{0} p(\mathbf{x}) \quad \text { for } \mathbf{x} \in B_{1}
$$




$$
\begin{aligned}
0+w_{0} w_{d}(\mathbf{x}) f(\mathbf{y}) h(\mathbf{z})+0+0=w_{0} f(\mathbf{y}) g(\mathbf{z})=w_{0} p(\mathbf{x}) & \text { for } \mathbf{x} \in B_{2} \\
0+0+w_{0} w_{d}(\mathbf{x}) q(\mathbf{y}) h(\mathbf{z})+0=w_{0} f(\mathbf{y}) g(\mathbf{z})=w_{0} p(\mathbf{x}) & \text { for } \mathbf{x} \in B_{3} \\
0+0+0+w_{0} f(\mathbf{y}) g(\mathbf{z})=w_{0} f(\mathbf{y}) g(\mathbf{z})=w_{0} p(\mathbf{x}) & \text { for } \mathbf{x} \in A
\end{aligned}
$$

But $w_{0} p(\mathbf{x})$ is the expected analog weight density at $\mathbf{x}$, so the method is unbiased.

A modification of the above method is to randomly sample only one of the three dxtran possibilities and multiply its weight by an additional factor of three because it is only sampled one third as often. This modification makes dxtran for double fluorescence similar to dxtran for single photon exits and also saves time by only following one dxtran sample rather than three. This modified method is shown in Fig. 42. Note that the "assumed probabilities" of one third are arbitrary. One can choose these probabilities as $p_{1}, p_{2}$, and $p_{3}$, in which case the weight multiplications are $p_{1}^{-1}, p_{2}^{-1}$, and $p_{3}^{-1}$ instead of the 3 shown in Fig. 42 . Test problems were successfully run with $p_{i}=1 / 3$, but I speculate that $p_{1}=.4, p_{2}=.4$, and $p_{3}=.2$ may be a better choice because the physical probability that both emitted photons reach the dxtran sphere is always less than half the probability that one photon reaches the dxtran sphere. Far from the dxtran sphere the probability that both photons reach the dxtran sphere is very small. If desired, $p_{1}, p_{2}$, and $p_{3}$ could be made to depend on the collision location, but this is beyond the scope of the present investigation.

\section{H. Summary of Supertrack Approach}

Non-Boltzmann tallies (such as pulse height tallies) can be done with the supertrack approach. The primary advantage of the supertrack approach is its direct connection with the physical transport process. This connection is philosophically useful because it means that new variance reduction techniques can be implemented relatively easily because the physics is easily understood and preserved with the supertrack approach. Additionally, effective use of variance reduction techniques is usually guided by the Monte Carlo practitioner's intuitive understanding of the natural transport process and the information supplied by short scoping calculations with the Monte Carlo code. The supertrack approach preserves the practitioner's ability to interpret the information supplied by Monte Carlo codes. The primary disadvantage is that the current transport algorithms all take a track-based approach and a supertrack-based approach will require significant alterations to existing Monte Carlo codes. If the current well-developed Monte Carlo codes did not exist, then there would be little question that the supertrack approach is more desirable than modified track-based approaches.

I have empirically verified that the supertrack approach can be implemented without excessive bookkeeping and produces unbiased estimates. In particular, generalizations of two standard Monte Carlo variance reduction techniques, splitting and dxtran, have been applied to supertracks. These were chosen as test variance reduction methods because they are the two methods that Guy Estes favored most. 


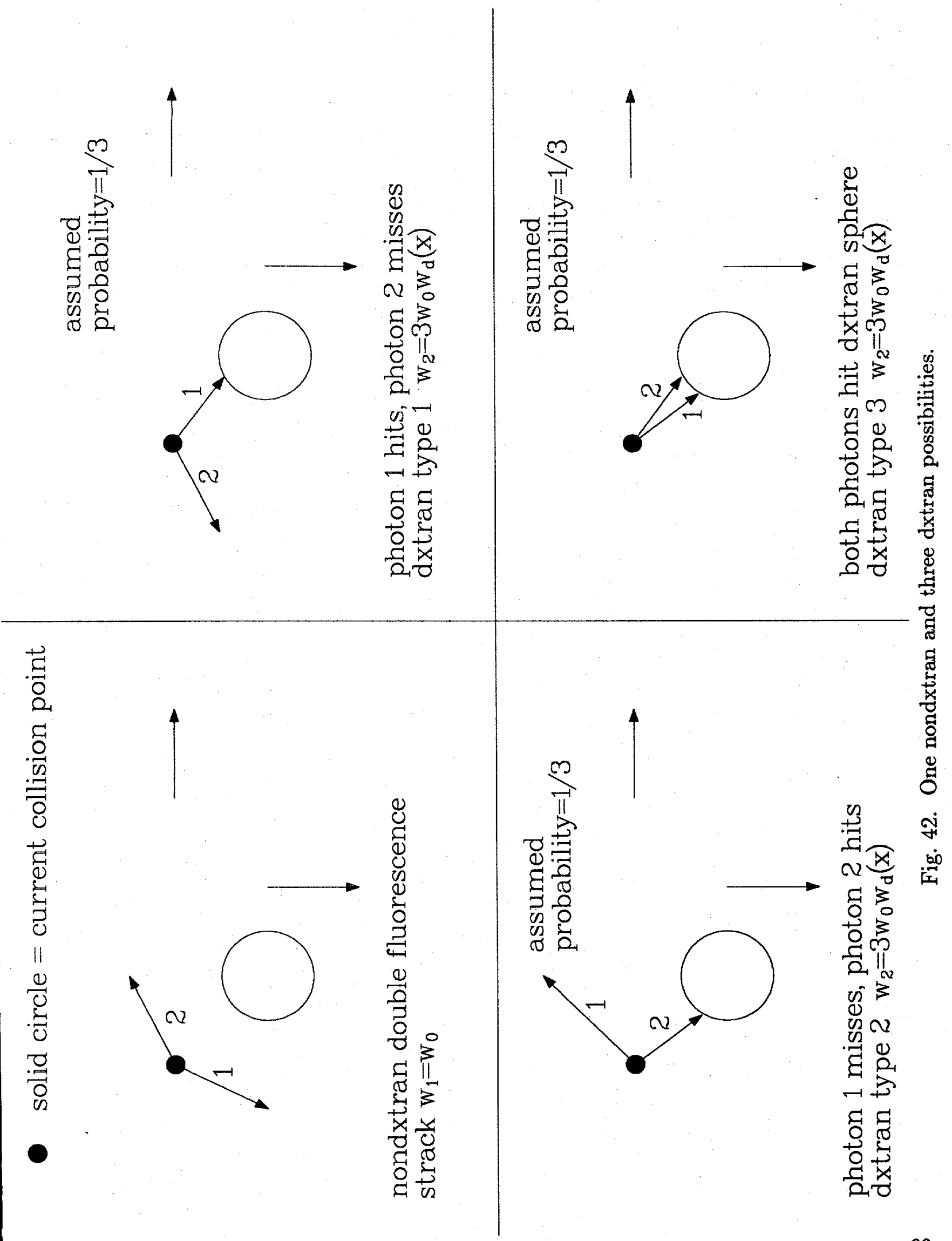




\section{ACKNOWLEDGMENTS}

I would like to thank Guy Estes and Art Forster for reading drafts of this report' and offering many useful suggestions.

\section{REFERENCES}

1. J. F. Briesmeister, editor, "MCNP - A General Monte Carlo Code for Neutron and Photon Transport Version 3A," Los Alamos National Laboratory manual LA-7396-M, Rev. 2 (September 1986). 